\title{
The biology and treatment of oligometastatic cancer
}

\author{
Diane K. Reyes ${ }^{1}$, Kenneth J. Pienta ${ }^{1,2}$ \\ ${ }^{1}$ Departments of Urology and Brady Urological Institute, and Oncology, The Johns Hopkins Medical Institutions, Baltimore, \\ MD, 21287, USA \\ 2Departments of Pharmacology and Molecular Sciences, and Chemical and Biomolecular Engineering, The Johns Hopkins \\ Medical Institutions, Baltimore, MD, 21287, USA
}

Correspondence to:

Kenneth J. Pienta, e-mail: kpienta1@jhmi.edu

Keywords: metastasis, therapy, tumor, spectrum theory, diaspora

Received: February 06, $2015 \quad$ Accepted: February 24, 2015

Published: April 13, 2015

\section{ABSTRACT}

Clinical reports of limited and treatable cancer metastases, a disease state that exists in a transitional zone between localized and widespread systemic disease, were noted on occasion historically and are now termed oligometastasis. The ramification of a diagnosis of oligometastasis is a change in treatment paradigm, i.e. if the primary cancer site (if still present) is controlled, or resected, and the metastatic sites are ablated (surgically or with radiation), a prolonged disease-free interval, and perhaps even cure, may be achieved. Contemporary molecular diagnostics are edging closer to being able to determine where an individual metastatic deposit is within the continuum of malignancy. Preclinical models are on the outset of laying the groundwork for understanding the oligometastatic state. Meanwhile, in the clinic, patients are increasingly being designated as having oligometastatic disease and being treated owing to improved diagnostic imaging, novel treatment options with the potential to provide either direct or bridging therapy, and progressively broad definitions of oligometastasis.

\section{INTRODUCTION}

Hellman first proposed the theory of oligometastases in 1995 as a sequel to the spectrum theory of cancer metastasis. Hellman hypothesized that the process of cancer metastases occurred along a continuum, from locally confined cancer to widely metastatic disease. Although the phenomenon of limited and treatable cancer metastases had been noted historically, Hellman and Weichselbaum proposed the term oligometastases, suggesting that in some patients with a limited number of clinically detectable metastatic tumors, the extent of disease exists in a transitional state between localized and widespread systemic disease. In this model local control (LC) of oligometastases would have the potential to yield improved systemic control, going against the dogma that control of oligometastatic disease would not have a therapeutic benefit since it represents a clinical manifestation of a few detectable lesions in the setting of widespread occult disease. Oligometastasis continues to be defined as a state of metastatic disease that is limited in total disease burden, usually by number of clinically evident or radiographic sites (either 1-3 or 1-5), and that is not rapidly spreading to more sites. The clinical implication of oligometastasis suggests that if the primary site (if still present) is controlled, or resected, and the metastatic sites are ablated (surgically or with radiation), there will be a prolonged disease-free interval, and perhaps even cure. As the understanding of the mechanisms underlying cancer metastasis have evolved, possible mechanisms for the oligometastatic state must be explained and examined within that context.

\section{Biological basis for oligometastasis}

Theories of metastasis

Metastasis is the cause of most cancer-related deaths [1]. In 1889, Stephen Paget [2] theorized that circulating tumor cells would "seed" to an amenable "soil", suggesting that metastasis was not a matter of chance. Five years later, [3, 4], in 1894, Halstead theorized that cancer was an orderly disease that progressed in a contiguous manner, by direct extension from the primary tumor through the lymphatics, to the lymph nodes, and then to distant sites. Halstead proposed that breast cancer metastasis was a progressive, 
anatomical process of contiguous seeding; his hypothesis supported the use of radical surgery and radiotherapy. Radical en bloc surgery, radical hysterectomy, and primary and regional irradiation for several tumor sites were all based on Halstead's theory of tumor spread [4]. James Ewing, in 1928, complemented the Paget and Halstead theories to propose that cancer cells grow at a particular site because they are directed by the direction of blood flow and lymphatics. [5]

The 'systemic' theory of metastasis, first suggested by Keynes [6] and further developed by Fisher [7], held that clinically apparent cancer was a systemic disease, and that small tumors were an early manifestation of systemic disease [8]. In this theory, nodal involvement was not part of an orderly contiguous extension but rather a marker of distant metastases. According to this theory, local control would not impact survival.

In contrast to the 'Halsted' theory and the 'systemic' theory, the 'spectrum' theory of cancer metastases, first described for breast cancer metastases in 1994, held that disease stage at the time of initial disease presentation fell into a spectrum ranging from indolent disease to widely metastatic, with the degree of clonal evolution determining the ability of the tumor to metastasize [9]. The spectrum theory was refined just one year later to describe the limited metastasis of any solid tumor and the term 'oligometastasis' was coined.

The spectrum theory conceptualized the entire range of metastatic competence, analogous to a diapason, which is the entire range of an instrument. To that end, the social sciences concept of a diaspora has recently been utilized to inform biologic understanding and therapeutic paradigms of cancer metastasis [10]. A diaspora refers to the scattering or movement of a population from its original homeland. In the case of systemic metastases, the diaspora resembles an imperial colonization in which the populations spread widely and eventually conquer the new host lands (aggressive cancer clones to multiple organs). Oligometastases resemble trading post diasporas, representing a limited number of outposts with limited growth potential (less aggressive cancer clones to few organs). (Table 1, Figure 1). Systemic versus oligometastatic diasporas may be dependent on the types of mutations present in the cancer cells (quality of the diaspora migrants), the quality of the original tumor site (factors in the homeland that cause the population to migrate), and the quality of the new hostland (factors that allow immigrants to establish and flourish) (Table 2).

\section{Biology of systemic and oligometastases}

It is now widely accepted that there are discrete steps in tumor metastasis. Initially there is a loss in cellular adhesion, followed by increased motility $>$ invasiveness of the primary tumor $>$ entry into and
Survival in the circulation $>$ entry into new organs $>$ eventual colonization of these organs $[11,12]$. Shortfalls at any stage of this metastatic progression could result in phenotypes of limited metastatic potential [13]. Gupta et al. described specific tumorigenic genes - initiation, progression, and virulence genes - that fulfilled specific roles in the metastatic cascade. Initiation genes afford a selective advantage to primary tumor cells to enter circulation. Progression genes fulfill rate-limiting steps for colonization. Virulence genes provide a selective advantage in cells to colonize a secondary site(s) [14]. As Weichselbaum and Hellman noted, this paradigm suggests "that there may be primary tumor cells with a limited capability in one or more of the necessary biological requirements for metastasis; thus proposing a possible biologic explanation of the origin of oligometastases" [15].

In 2000 and updated in 2011, Hanahan and Weinberg proposed the, widely accepted, 'hallmarks of cancer' [16]. The original cancer hallmarks consisted of six transformations in cellular physiology that allow cells to survive, proliferate and disseminate, and together support carcinogenesis. The update posited that underlying the original hallmarks were two 'enabling hallmarks': 'genome instability and mutation' and 'tumor-promoting inflammation'. Additionally, the update proposed two new 'emerging hallmarks': 'deregulation of cellular energetics' and 'avoidance of immune destruction' [17]. The exact sequence in which the transformations occur, and thus the appearance of the 'hallmarks' (selfsufficiency in growth signals $\leftrightarrow$ insensitivity to antigrowth signals $\leftrightarrow$ tissue evasion and metastasis $\leftrightarrow$ limitless replicative potential $\leftrightarrow$ sustained angiogenesis $\leftrightarrow$ evading apoptosis) can vary throughout the course of progression. Despite the ordering, collectively, the hallmarks can terminate into a cancer. However, the ordering and the degree of specific hallmarks, may potentially allow for a subsequent oligometastatic state. For example, cancer cells that lack the hallmarks to actively metastasize still may be able to slough into the circulation and inefficiently establish metastases. Other cells may be less efficient at proliferation, establishing slow growing metastases. Warburg (1956) suggested that "We may have cells which indeed look like cancer cells but are still energetically insufficient... such cells which are clinically not cancer cells, have lately been found not only in the prostate, but also in the lungs, kidney, and stomach of elderly persons such cells have been referred to as "sleeping cancer cells" [18].

\section{Preclinical models of oligometastasis}

Traditional clinicopathologic factors are inadequate when attempting to define the potential underlying biology of oligometastases. Several investigations have demonstrated the marked genetic and epigenetic 
Table 1: A comparison of migrants, diaspora, and the spectrum of cancer metastases

\begin{tabular}{|c|c|c|c|}
\hline \multicolumn{2}{|l|}{ Social Demography } & \multicolumn{2}{|l|}{ Cancer Demography } \\
\hline Imperial Diaspora & Trading Post Diaspora & $\begin{array}{l}\text { Trading Post Diaspora } \rightarrow \\
\text { Oligometastasis }\end{array}$ & $\begin{array}{l}\text { Imperial Diaspora } \rightarrow \\
\text { Cancer metastasis }\end{array}$ \\
\hline $\begin{array}{l}\text { Large populations from a } \\
\text { single homeland }\end{array}$ & $\begin{array}{l}\text { Small population from a } \\
\text { single homeland }\end{array}$ & $\begin{array}{l}\text { Migrated from primary } \\
\text { cancer in passive manner }\end{array}$ & $\begin{array}{l}\text { Dispersed from a primary } \\
\text { cancer in an active manner }\end{array}$ \\
\hline $\begin{array}{l}\text { Settle multiple countries in } \\
\text { aggressive manner }\end{array}$ & $\begin{array}{l}\text { Settle in few countries while } \\
\text { avoiding upsetting host } \\
\text { country }\end{array}$ & $\begin{array}{l}\text { Mild hypoxia and unlimited } \\
\text { nutrients; Home niche } \\
\text { conditions do not cause } \\
\text { evolutionary clonal pressure }\end{array}$ & $\begin{array}{l}\text { Hypoxia and lack of } \\
\text { nutrients cause pressure to } \\
\text { leave primary; Evolving } \\
\text { home niche conditions cause } \\
\text { undifferentiated, aggressive } \\
\text { clones. }\end{array}$ \\
\hline $\begin{array}{l}\text { Host country may or may not } \\
\text { be receptive }\end{array}$ & $\begin{array}{l}\text { Host country may or may not } \\
\text { be receptive }\end{array}$ & $\begin{array}{l}\text { Target organ may or may not } \\
\text { be receptive }\end{array}$ & $\begin{array}{l}\text { Target organ may or may not } \\
\text { be receptive }\end{array}$ \\
\hline $\begin{array}{l}\text { Group maintains collective } \\
\text { memory of their homeland } \\
\text { and culture }\end{array}$ & $\begin{array}{l}\text { Group maintains collective } \\
\text { memory of their homeland } \\
\text { and culture }\end{array}$ & $\begin{array}{l}\text { Pathologists can identify } \\
\text { where a cancer cell } \\
\text { originated }\end{array}$ & $\begin{array}{l}\text { Pathologists can identify } \\
\text { where a cancer cell } \\
\text { originated }\end{array}$ \\
\hline $\begin{array}{l}\text { Often assimilate the new } \\
\text { homeland }\end{array}$ & $\begin{array}{l}\text { Survive as distinct } \\
\text { communities }\end{array}$ & Few distinct metastases & $\begin{array}{l}\text { Multiple metastases as } \\
\text { distinct masses }\end{array}$ \\
\hline $\begin{array}{l}\text { Relationship with host } \\
\text { country is uneasy and } \\
\text { degenerates over time }\end{array}$ & $\begin{array}{l}\text { Relationship with host } \\
\text { country may be uneasy but is } \\
\text { maintained over time }\end{array}$ & $\begin{array}{l}\text { Immune system may not see } \\
\text { a threat }\end{array}$ & $\begin{array}{l}\text { Immune system tries to } \\
\text { destroy the cancer cells }\end{array}$ \\
\hline $\begin{array}{l}\text { Tied to the homeland by } \\
\text { exchange of resources }\end{array}$ & $\begin{array}{l}\text { Tied to the homeland by } \\
\text { exchange of resources }\end{array}$ & $\begin{array}{l}\text { Limited need for outside } \\
\text { resources from homeland; } \\
\text { fewer cells trafficking }\end{array}$ & $\begin{array}{l}\text { Multiple cell-type trafficking } \\
\text { trafficking of resources/info }\end{array}$ \\
\hline
\end{tabular}

Table adapted from Pienta et al. Clin Can Research, 2013 [10]

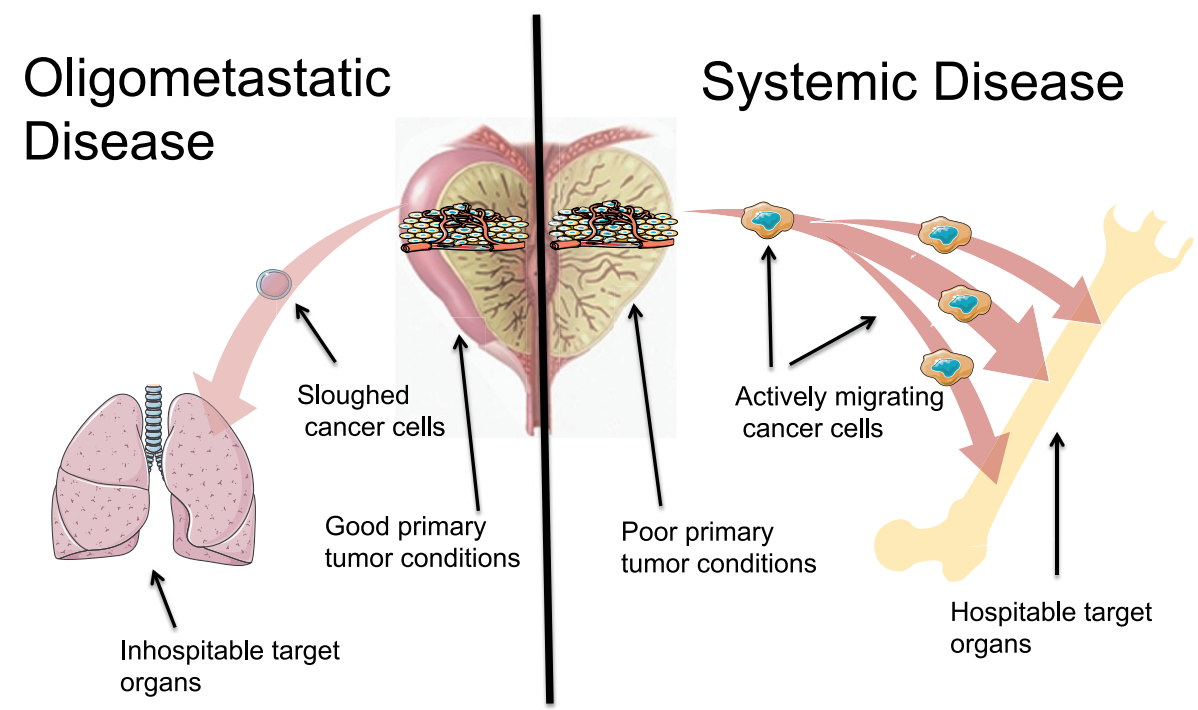

Figure 1: Oligometastatic disease versus systemic disease. (left) Oligometastatic disease. Metastatic growth potential is limited. This could be (a) secondary to due to environmental conditions in the primary tumor forestalling evolutionary clonal pressure, (b) cancer cells that slough out of the primary tumor that do not have the properties necessary to survive the circulation and invade into target organ sites, and/or (c) the cancer cells land in inhospitable target organs. (right) Systemic disease. Widespread metastatic growth potential is unlimited. This could be (a) secondary to due to environmental conditions in the primary tumor creating many undifferentiated, aggressive clones, (b) cancer cells that actively migrate out of the primary tumor that have the properties necessary to survive the circulation and invade into target organ sites, and/or (c) the cancer cells land in hospitable target organs. 


\section{Table 2: Factors that define the rate and success or failure of oligometastases and systemic metastases}

\begin{tabular}{|c|c|c|c|}
\hline Cancer dispersion & $\begin{array}{l}\text { Quality of primary } \\
\text { microenvironment }\left[{ }_{\Delta} Q_{0} I_{\Delta} t\right]\end{array}$ & $\begin{array}{l}\text { Fitness of migrant cancer } \\
\text { cells }\left(\mathrm{E}_{\mathrm{Fn} \Delta t}\right)+\left(\mathrm{M}_{\mathrm{Fn} \Delta t}\right)+\left(\mathrm{S}_{\mathrm{Fn} \Delta t}\right)\end{array}$ & $\begin{array}{l}\text { Quality of metastatic sites } \\
\left(\mathrm{H}_{\mathrm{Qn \Delta t}_{\mathrm{t}}}\right)+\left(\mathrm{H} \mathbf{2}_{\mathrm{Qn \Delta t}}\right) \ldots\end{array}$ \\
\hline Oligometastasis & ${ }_{\Delta} Q_{0}=$ little change & {$\left[\left(\mathrm{E}_{\mathrm{Fn} \Delta t}\right)>\left(\mathrm{M}_{\mathrm{Fn} \Delta t}\right)\left(\mathrm{S}_{\mathrm{Fn} \Delta t}\right)\right][\mathrm{TR}]$} & {$\left[\left(\mathrm{H} 1_{\mathrm{Qn}}\right)+\left(\mathrm{H} 2_{\mathrm{Qn})}\right]=\right.$ low } \\
\hline Widespread metastasis & ${ }_{\Delta} Q_{O}=$ decreasing & {$\left[\left(\mathrm{E}_{\mathrm{Fn} \Delta t}\right)<\left(\mathrm{M}_{\mathrm{Fn} \Delta t}\right)\left(\mathrm{S}_{\mathrm{Fn} \Delta t}\right)\right][\mathrm{TR}]$} & {$\left[\left(\mathrm{H} 1_{\mathrm{Qn})}\right)+\left(\mathrm{H} 2_{\mathrm{Qn}}\right)=\right.$ high } \\
\hline
\end{tabular}

Primary microenvironment quality: ${ }_{\Delta} Q_{O}=$ the changing quality $\left({ }_{\Delta} Q\right)$ of the primary cancer site over time $\left({ }_{\Delta} \mathrm{t}\right)$. The quality of the primary microenvironment is dependent on multiple factors, including $\mathrm{pH}$, oxygenation, amount of nutrients, interaction with supporting host cells, and the quality of the immune response. Primary nonlethal, epithelial cancer cells in a highly vascularized environment with rich nutrients are presumed to be less likely to evolve to a more aggressive clone by undergoing an epithelial to mesenchymal transition (EMT) and leave the primary over time. As the quality of the environment decreases, it is likely that the generation of lethal, mesenchymal clones increases.

Fitness of migrant cancer cells: $\mathrm{E}_{F n \Delta t}=$ the number $(n)$ and fitness $(F)$ of passively shed epithelial cancer cells (E) over time $(\Delta t)$. This represents the likelihood that a cancer cell passively shed into the circulation will survive transport to a target organ. $\mathrm{M}_{F n \Delta t}=$ the number $(n)$ and fitness $(F)$ of actively emigrant cancer cells $(\mathrm{M})$ over time $(\Delta t) . \mathrm{S}_{\mathrm{Fn} \Delta \mathrm{t}}=$ number $(n)$ and fitness $(F)$ of cancer stem cells $(\mathrm{S})$ over time. It is likely that the fitness of a passively shed cell is less than a migrating mesenchymal cell or stem cell that actively exits the primary tumor through the lymphatics, nerves, or circulation. Fitness depends on many variables, including EMT state, stemness, ability to secrete MMPs, ability to avoid anoikis, etc.

Quality of metastatic sites: $\mathrm{H}_{Q n}=$ the quality $(Q)$ of the target organ or host-land sites $(\mathrm{H} 1, \mathrm{H} 2 \ldots)$. Migrating cancer cells will land in multiple sites $(n)$ within different target organs in order to immigrate. Success depends on the quality of the soil of each of these microenvironments as well as the host immune response.

Table adapted from Pienta et al. Clin Can Research, 2013, [10]

heterogeneity present in metastatic cancer sites within the same patient [19-22]. These studies demonstrate that cancer cells at different sites within a patient can have varied malignant potential [23-25]. Preclinical models of tumors with varying degrees of metastatic potential, including low metastatic potential exist. Using a cell line derived from B16F1 melanoma, Fidler et al. [26] found that variant metastatic cells pre-exist in a heterogeneous primary tumor as opposed to originating through adaption during metastasis from an otherwise homogenous primary tumor. This finding was advanced in later work that showed the KHT sarcoma line demonstrated similar heterogeneity whether grown in vitro or in vivo, which suggested that clonal variation seen in vitro derived from heterogeneity present in the primary tumor [27]. As an extension of this work in the KHT sarcoma line, it was demonstrated that effective metastatic variants developed at a high rate with low frequency, as opposed to the more frequent and stable subpopulations of metastatic variants [28]. In comparing B16 cell lines, Cillo et al. showed that the more highly metastatic, and less genetically stable cell line, generated increased metastatic variants corresponding to increased chemotherapy resistance [29]. Numerous in vitro studies and analyses of animal models have indicated that cells isolated from metastases differ greatly - both genetically and phenotypically — from cells isolated from their parental primary tumors [30]. The preclinical models point toward variation in individual tumor cells' metastatic potential, which supports the concept of oligometastases [15].
Given that stochastic models have been used to predict biologic phenomena, a Bayesian model has been proposed to predict the chance of occult metastases in the presence of detectable oligometastases [31]. Using the size and number of metastases, the proposed model inferred, (1) that the probability of occult metastases may increase substantially with minor increases in metastatic potential and (2) that extended disease-free periods were predictive of a substantial decrease in additional occult disease. Although compelling, such models are in their infancy and as yet remain in pre-clinical testing where the host, tumor and experimental factors are controlled. [31]

\section{Clinical evidence of oligometastasis}

Evidence for the evolution of the oligometastatic phenotype comes from various clinical and pre-clinical sources [26, 32-34]. Recent studies of the molecular biology of renal cell cancer metastasis have implied biologic differences between less and more aggressive metastases, as well as between fewer and multiple metastases. In order to better determine which patients presenting with localized RCC harbor an aggressive tumor and may not benefit from surgery, Kosari et al. using gene expression profiling, found gene expression alterations associated with an aggressive tumor and metastatic potential in the primary tumor [35]. From a cohort of 20 resected pulmonary metastases taken from 18 patients, Wuttig et al demonstrated the predictive potential of identified gene signatures, when comparing 
disease-free intervals (DFI) and number of metastases, both of which are predictive of prognosis in metastatic RCC (mRCC). There were 306 differentially expressed genes in comparing DFI $\geq 5$ years and DFI $\leq 9$ months, and 135 differentially expressed genes in comparing multiple metastases $(\geq 16)$ and few metastases $(\leq 8)$. [36]

In colorectal cancer, there is growing evidence that liver-limited disease is a distinct biological cohort that may benefit from aggressive management. While only a minority of patients are technically resectable, approximately $40 \%$ of patients with resected liver limited disease are alive 5 years after diagnosis compared with less than $1 \%$ for those with disseminated disease. [37] There is genetic evidence that patients undergoing hepatic resection for metastatic cancer had a different disease than those who did not [37]. For example, it was noted that BRAF V600E mutant tumors, which were typically associated with aggressive biology, rarely came to liver resection $[38,39]$. In addition, novel chromosomal aberrations have been identified that are associated with intra- and extra-hepatic recurrence after liver resection [40].

MicroRNAs, small non-coding RNA known to regulate tumor proliferation and apoptosis, are frequently dysregulated in cancer and metastasis [41-43]. MicroRNA profiling has shown a possible method to distinguish patients with oligometastases from those with polymetastatic disease. Examples of pro-metastatic microRNAs include microRNA-10b (upregulated in primary breast tumors that had metastasized), microRNA-21 (correlated with advanced stage, incidence of metastases, and poor outcomes in breast and pancreatic tumors), and microRNA373/520c (increased expression in breast metastases) [43]. MicroRNA-210, a known transcriptional target of the HIF-1 $\alpha$ signaling pathway, was elevated in sera from patients with metastatic castrate-resistant prostate cancer, as compared to controls, and was correlated with treatment response as assessed by change in PSA [41]. Lussiter et al. found microRNA-200c was associated with polymetastatic progression in a oligometastatic cell line, derived from patients treated with high-dose radiotherapy, tested in a xenograft model [44]. The investigators then stratified patients with resected pulmonary oligometastases into subgroups, based on high-risk versus low-risk of further metastatic progression. Differential microRNA expression patterns were identified between these two groups (high rate of progression ( $n=16$ prioritized microRNAs) and low rate of progression ( $n=32$ prioritized microRNAs) and, in an independent dataset, the expression patterns were associated with risk of progression and decreased overall survival. [8] Most recently, Uppal et al. identified three microRNAs overexpressed in clinical metastasis samples from patients with limited metastatic disease. MicroRNA127-5p, microRNA-544a, and microRNA-655-3p were shown to limit, but not fully inhibit, metastasis in a model of breast cancer lung colonization [45].

\section{Controversies surrounding the treatment of oligometastatic disease}

Hellman theorized that, 1) whereas some tumors were destined to remain localized, 2) other tumors, as they increased in size, acquired an increasingly greater metastatic phenotype, suggesting at an early stage these tumors seeded distant sites with clones that had not reached full metastatic potential, and finally, 3) that some tumors already had occult distant dissemination at the time they are diagnosed. He also proposed that metastatic potential was not only directed by the tumor phenotype, but that it was also influenced by the tumor's location, venous drainage, and host factors $[4,46]$. Based on Hellman's theory, later scientists further theorized that tumors in the oligometastatic disease state were tumors early in their evolution of metastatic progression; therefore they produced metastases that were limited in number and location. These data support the presumption of a temporal evolution with an intermediate stage of limited metastatic capacity, where oligometastatic tumors may not have acquired the broad array of genetic changes required to develop widespread metastases [14, 47, 48].

The clinical implication of the oligometastatic state is that locally ablative therapies, given with the intent of targeting sites of clinically evident metastatic disease could result in long-term survival or cure [15, 49]. Treatment of oligometastatic disease may also result in decreased overall tumor burden, decreasing morbidity and increasing survival. These arguments are opposed by the concept that clinical metastases are evidence of systemic disease and locally directed treatment will not alter the natural history of the disease course within a patient. In this scenario, only systemic therapy may be beneficial. Indeed, oligometastatic treatment paradigms are controversial (due to the limited data available) $[4,34,50,51]$. Without randomized studies, it is impossible to know if treatment of oligometastatic disease helps the patient. In addition, oligometastatic disease may represent indolent disease that does not require potentially toxic treatments $[4,34,50,51]$.

Patients are increasingly being diagnosed with oligometastatic disease due to the advent of sensitive imaging technologies as well as effective therapies that are allowing patients to live longer with the diagnosis of cancer [34, 52]. In addition, the fact that novel treatment options with acceptable safety profiles, such as stereotactic radiation, cryoablation, and minimally invasive surgery, are available to treat limited metastases, has led to a renewed interest in treating oligometastatic disease. Treatment of oligometastatic disease not only has the potential to prevent further evolution of genetically unstable clones and metastatic spread, it may improve overall disease control and delay more toxic systemic treatment $[13,34,53,54]$. Finally, the definition of oligometastases had gradually evolved (Table 3 ), which further inflates the increasing population of patients 
Table 3: Definitions of Oligometastasis

\begin{tabular}{|c|c|c|}
\hline Terms & Definition & Reference \\
\hline Oligometastasis & $\begin{array}{l}\text { "...metastases (from tumors early in the chain of progression) limited in } \\
\text { number and location because the facility for metastatic growth has not been } \\
\text { fully developed and the site for growth is restricted..." }\end{array}$ & [46] \\
\hline Oligometastatic disease & $\begin{array}{l}\text { Solitary or few detectable metastatic lesions that are usually confined to a } \\
\text { single organ }\end{array}$ & {$[50]$} \\
\hline Oligometastases & $\begin{array}{l}\text { Due to limited metastatic competence and does not occur following } \\
\text { otherwise successful systemic treatment. New metastases in this situation, } \\
\text { albeit even limited, is likely to have more extensive malignant capabilities } \\
\text { that were somehow spared from eradication by therapeutic means, or from } \\
\text { the development of resistant clones }\end{array}$ & [15] \\
\hline Induced oligometastases & $\begin{array}{l}\text { Occurs when widespread micrometastatic disease is mostly eradicated by } \\
\text { systemic chemotherapy but drug resistant clones are left behind, or tumor } \\
\text { foci is located in a site not accessed by chemotherapy }\end{array}$ & {$[4]$} \\
\hline Oligorecurrence & Limited metastases in the presence of a controlled primary lesion & [195] \\
\hline Sync-oligometastases & $\leq 5$ metastatic or recurrent lesions in the presence of active primary lesions & {$[196]$} \\
\hline Synchronous oligometastasis & $\begin{array}{l}\text { Oligometastatic disease is detected at the time of diagnosis of the primary } \\
\text { tumor, therefore there is an active primary tumor }\end{array}$ & [196] \\
\hline Metachronous oligometastasis & $\begin{array}{l}\text { Development of oligometastatic disease after treatment of the primary } \\
\text { tumor; interval for classification of metachronous versus synchronous is not } \\
\text { standardized; between Controlled primary lesion except for concomitant } \\
\text { primary and distant recurrence }\end{array}$ & [196] \\
\hline Oligoprogression & $\begin{array}{l}\text { Progression of a limited number of metastatic deposits, while remaining } \\
\text { metastases are controlled with systemic therapy }\end{array}$ & [197] \\
\hline $\begin{array}{l}\text { Oligometastasis (specific to } \\
\text { prostate cancer) }\end{array}$ & $\begin{array}{l}\text { Rising PSA following primary therapy, with oligometastasis on imaging, in } \\
\text { whom local treatment (surgical metastasectomy (usually LN dissection), or } \\
\text { SBRT for bony mets or LN recurrence) is required to defer initiation of ADT }\end{array}$ & {$[54]$} \\
\hline $\begin{array}{l}\text { Oligometastasis (specific to } \\
\text { prostate cancer) }\end{array}$ & $\begin{array}{l}\text { Castrate resistant prostate cancer with a rising PSA and oligometastasis on } \\
\text { imaging, in whom local treatment (surgical metastasectomy (usually LN } \\
\text { dissection), or SBRT for bony mets or LN recurrence) may allow deferral of } \\
\text { ADT }\end{array}$ & {$[54]$} \\
\hline
\end{tabular}

Abbreviations: $\mathrm{LN}=$ lymph node; $\mathrm{SBRT}=$ stereotactic body radiation therapy; mets=metastases; $\mathrm{ADT}=$ androgen deprivation therapy; PSA=prostate-specific antigen

diagnosed with oligometastasis. In the absence of data to guide decisions, treatment of oligometastatic disease may be seen as a quality-of-life oriented approach, choosing personalized treatments with a reasonable risk to benefit ratio and taking into account the patient's own attitude in guiding them toward more or less, intensive therapy [50].

\section{Therapeutic options for oligometastases}

\section{Radiation therapy}

While much of the literature supporting the oligometastatic states is within the surgical literature, there is an increasing body of literature describing the use of stereotactic body radiotherapy (SBRT) and stereotactic radiosurgery (SRS), in addition to conventional fractionated radiotherapy techniques [51]. SBRT is a noninvasive method of delivering high doses of radiation to ablate a target lesion while sparing the neighboring normal tissue, thus reducing long-term effects of radiation on the non-malignant tissues. The radiation is delivered from many beams originating from multiple directions that converge on the target site. [55] Through improved targeting and management of tumor motion, SBRT may improve tumor control and reduce treatment-related toxicity, as compared to conventional fractionated RT. Improved radiation targeting allows for higher-dose, hypofractionated, more efficient treatment regimens that can be delivered within narrow margins sparring adjacent organs. [56] 'Hypofractionation' is the delivery of large doses of radiation over a shorter time period as compared 
to conventional radiation fraction sizes. Therapy can generally be completed in 1-5 sessions, as compared to conventional radiation therapy that is delivered in smaller doses 5 days/week over $\geq 6$ weeks. [55]

SBRT can be used to manage oligometastatic disease presentations that would be associated with added morbidity if managed by surgery, such as deep-seated or osseous lesions. Patients who are poor surgical candidates may often be treated with SBRT, given that it is noninvasive and has a modest morbidity profile. Whereas surgery, when used to manage oligometastatic disease, tends to be seen as the gold standard as it allows for pathologic evaluation and assessment of the surgical margins. In addition, lesions greater than 7-8 cm and those difficult to target with SBRT, are better left for surgical management. [55]

Although traditionally radiation therapy was thought to be immunosuppressive, there is increasing pre-clinical and clinical evidence that high dose, hypofractionated radiation -SBRT- may reverse antitumor immunity via CD8+ T-cells and cellular stress signals. [55] Although uncommon, the abscopal effect (regression in tumors distant to the targeted field of radiation) is an example of recovery of anti-tumor immunity following RT [57]. While the exact mechanism is unknown, it has been proposed that the radiation effect may result in the release of anti-tumor proliferative antigens and cytokines. Matzinger [58] theorized a 'danger model', which suggested that the immune system is stimulated by injured tissues rather than 'non-self', therefore damaged tissues in irradiated sites may stimulate the immune system. Lee et al. [59] demonstrated in a murine model that hypofractionated, high dose RT may possibly reverse T-cell unresponsiveness to primary and metastatic tumors. More specifically, considering that local RT to a tumor may modify its microenvironment by producing inflammatory cytokines that may increase its immune responsiveness, Lugade et al. demonstrated that IFN- $\gamma$ (a cytokine), following RT, promotes $\mathrm{T}$ cell function [60]. It is thought that a tumor has ongoing crosstalk with the immune system- from one end of a spectrum where the immune system eliminates the tumor, to tumor-immune system equilibrium (a subclinical tumor), until selective pressure from the immune system stimulates the evolution of tumor cells resistant to the immune system (clinically detectable). A way to think of how RT may promote immune recovery is that the irradiated tumor is converted into a 'vaccine' that promotes tumor specific $\mathrm{T}$ cells to bestow immune memory against non-treated tumors. [57]. Thus, an emerging advantage of SBRT is the possibility that it may be exploited to benefit the immune system.

\section{Surgery}

Historically, most of the surgical data in regards to oligometastatic disease is centered on hepatic resection. [37]. Perioperative mortality related to hepatic resection has decreased from $20 \%$ (before 1980) to $1 \%$. Recent improvement in overall survival following hepatectomy, are likely from improvements in patient selection (shift in definition of resectability to new criteria based on whether a macroscopic and microscopic complete resection of the liver lesion as well as complete resection of any extrahepatic disease), surgical technique, and more effective adjuvant therapy. The use of portal vein embolization and of neoadjuvant chemotherapy have also expanded the population of patients who are eligible for resection. [61] In a recent series of patients having undergone hepatic resection, despite progression on chemotherapy, the 5-yr survival was 53\% [51].

Secondary resection remains a worthwhile therapeutic goal; patients brought to resection by systemic therapy enjoy comparable long-term survival to patients who had resectable disease at the time of presentation, and far superior to those receiving palliative systemic chemotherapy [62]. 10-20\% of patients that develop colorectal liver metastases present with, or are converted by systemic treatment to, an oligometastatic state defined as metastatic lesions that are limited in number and involving only a single organ. This type of disease is potentially amenable to local therapeutic modalities, of which hepatic resection is the most effective. [51]

\section{Cryotherapy}

Cryotherapy has been utilized in multiple settings for the ablation of metastatic disease [63, 64]. In the largest series published to date, Littrup and colleagues treated a total of 251 oligometastatic tumors from multiple primary cancers in 126 patients [65]. Sites of treatment included retroperitoneal, superficial, intraperitoneal, bone, and head and neck; average diameter of tumors was $3.4 \mathrm{~cm}$. At 11 months average follow-up (range, 0-82 months), a 10\% total recurrence rate (26 of 251) was noted; three occurred within the ablation zone, for a local progression rate of $1.2 \%$. The average time to recurrence was 4.9 months, and, at 21 months, the initial ablation zone had reduced in volume by $93 \%[65]$.

\section{Treatment of oligometastases in different cancers}

Anecdotal reports of tumors with limited metastases, having undergone treatment to the metastases and had long-term response date back to the 1930's [66]. Several studies, with a variety of endpoints and meeting different levels of evidence criteria, have been performed in a variety of cancers to treat oligometastatic disease. For each clinical study reviewed, we considered the definition of oligometastasis that was used, the therapy given, and sample size. In order to provide an ordinal categorization to assess the strength of the study designs and of the study endpoints, we used the National Cancer Institute Levels of Evidence for Adult and Pediatric Cancer Treatment Studies ranking system to assess each study [67]. In this system, the strength of the studies 
in descending order is: randomized controlled trials (double-blinded): Level $1 \mathrm{i}>$ randomized controlled trials (non-blinded): Level 1 1 ii $>$ non-randomized controlled clinical trials: Level $2>$ case series (population-based, consecutive series): Level $3 \mathrm{i}>$ case series (consecutive cases (not population-based)): Level 3ii > case series (nonconsecutive cases): Level 3iii. The system ranks the study endpoints in descending order of strength as follows: total mortality: Level $\mathrm{A}>$ cause-specific mortality: Level B > quality of life: Level C > event-free survival: Level Di $>$ disease-free survival: Level Dii $>$ progression-free survival: Level Diii $>$ tumor response rate: Level Div.

\section{Breast cancer}

Metastatic breast cancer (MBC) at diagnosis constitutes $3.5-7 \%$ of all new breast cancers [68], while oligometastases comprises $1-3 \%$ of the total MCB population [50]. Traditionally MBC patients were managed primarily with systemic modalities, with limited local treatments given only for palliative intent.

We searched PubMed using the terms 'breast oligometastases', 'breast oligometastasis', and 'oligometastatic breast cancer'; results were as follows, $n=20, n=5$, and $n=51$, respectively. We omitted reviews (including special features), studies including mixed primary tumors, case reports, studies not in the English language, studies not focused on oligometastatic breast cancer, and a survey. There were seven clinical studies remaining; oligometastases held six definitions and each study used a different therapy (Table 4).

The evidence for treatment of oligometastases in breast cancer is weak based on the study designs. There were two prospective studies $[69,70]$, however they were both nonconsecutive case series (Level 3iii) as were the remaining studies, with the exception of one consecutive case series (Level 3ii) [71]. There was a conglomerate of therapies given for patients diagnosed with oligometastases using various definitions. The fact that overall survival endpoints were reported in all studies was helpful (Level A, $n=7$ studies) [69-75], however, they were not always significant - stronger study designs may have shown whether the survival endpoints were significant. Overall survival (OS) was reported at various intervals making comparisons between studies difficult: median (42.2 months) [69], 20-yr (34\%) [71, 72], 5-yr (62\%) [75], 4-yr (59\%) [70] and 3-yr (73\% [73] and 39-57\%) [74]).

\section{Lung cancer}

Non-small cell lung cancer (NSCLC) is the leading cause of death worldwide with $>50 \%$ of patients having metastatic disease at diagnosis [76, 77]. The primary treatment for most patients with metastatic NSCLC is palliative chemotherapy (CT), which results in median survivals of 8-11 months [78]. However, multiple studies have demonstrated a subset of long-term survivors with
OMLC [76, 77]. Additionally, curative outcomes were documented in patients with treated adrenal metastases [79].

We searched PubMed using the terms 'lung oligometastasis', 'lung oligometastases', and 'oligometastatic lung cancer'; results were as follows, $n=14, n=90$, $n=123$, respectively. We omitted reviews, mixed primary tumors, studies not focused on oligometastatic lung cancer, case reports, editorials, studies not in the English language, surveys, commentaries, cost analyses, studies including less than 10 patients, and studies we were unable to retrieve. There were twenty clinical studies remaining (Table 5); oligometastasis held 17 different definitions and each study had a different treatment paradigm.

The evidence for treatment of oligometastases in lung cancer was weak-to-moderate based on the study designs. There were two nonrandomized controlled clinical trials (Level 2) [80, 81], however the sample sizes were small ( $n=26$ and $n=39$, respectively). There was one large study $(n=2176)$, although it was retrospective and a nonconsecutive case series (Level 3iii) [76]. Of the remaining 17 studies, two were population-based consecutive case series (Level 3i) [82, 83], two were non population-based consecutive case series (Level 3ii) $[84,85]$, and twelve were nonconsecutive case series (Level 3iii) [76, 86-97], one of which was prospective [98]. Therapies given varied not only among the studies but within most of them as well, for patients with diversely defined oligometastases. Overall survival endpoints (level A) were reported in all but one study [90], however stronger study designs would have shown whether the survival endpoints were significant. OS was reported as MOS (11.7 [82], 17 [94], 20 [83], 26.4 [92], and 41months [91] and at four intervals in the remaining studies: 5-year (29.4\% [84], 8.3-86\% [76], 44.7\% [98], 38\% [96], 36\% [86], and 24\% [87]), 3-year (17.5\% [81], 25\% [85], and $15.8 \%$ [97]), 2-year (24\% [93]and 38\% [89]), 18-month $(52.9 \%)$ [88] and 1-year (67\% [80]and 75\% [95]).

\section{Melanoma}

Approximately $30 \%$ of patients with melanoma will develop metastases. The 5-yr survival of stage IV melanoma is about $5 \%$.

We searched PubMed using the terms 'melanoma oligometastasis', 'melanoma oligometastases' and 'oligometastatic melanoma'; results were as follows, $n=5$, $n=5$, and $n=15$, respectively. We omitted reviews, studies including mixed primary tumors, and studies not focused on oligometastatic melanoma. An additional search through the bibliographies of the review papers allowed us to retrieve one additional clinical study for review, therefore we reviewed two clinical studies (Table 6). The two studies differed in their therapies, which included resection [99], and SRS followed by $+/-$ immunotherapy [100], and definitions of oligometastases.

The evidence for treatment of oligometastases in melanoma is weak based the two, retrospective 
Table 4: Oligometastatic breast cancer

\begin{tabular}{|c|c|c|c|c|c|c|c|}
\hline $\begin{array}{l}\text { 1st Author, } \\
\text { year [Ref] }\end{array}$ & $\begin{array}{c}\text { Strength } \\
\text { of evidence } \\
\text { (study } \\
\text { design / } \\
\text { endpoint) }\end{array}$ & $\begin{array}{l}\text { Prospective } \\
\text { (P) or } \\
\text { Retros- } \\
\text { pective (R) }\end{array}$ & $\begin{array}{l}\text { Sample } \\
\text { size }\end{array}$ & $\begin{array}{l}\text { Definition- } \\
\text { Oligometastases }\end{array}$ & Therapy & Endpoint & Conclusion \\
\hline $\begin{array}{l}\text { Kobayashi, } \\
2012[71]\end{array}$ & $3 \mathrm{ii} / \mathrm{A}$ & $\mathrm{R}$ & 75 & $\begin{array}{l}\text { 1-2organs with met } \\
\text { lesions, } \leq 5 \text { lesions/ } \\
\text { organ, } \leq 5 \mathrm{~cm} \text { lesion } \\
\text { diameter }\end{array}$ & $\begin{array}{l}+/-\mathrm{CT} \text {, then } \\
+/- \text { local } \\
\text { therapy }+\mathrm{CT}\end{array}$ & $\begin{array}{l}10 y r \text { OS- } \\
59.2 \% ; 20 y r \\
\text { OS } 34.1 \%\end{array}$ & $\begin{array}{l}\text { Prognosis of } \\
\text { OMBC superior to } \\
\text { that of } \mathrm{MBC}\end{array}$ \\
\hline $\begin{array}{l}\text { Bojko, } \\
2004 \text { [69] }\end{array}$ & 3iii / A & $\mathrm{P}$ & 48 & $\begin{array}{l}1 \text { organ with } 1-\text { few } \\
\text { met lesions }\end{array}$ & $\begin{array}{l}\text { Surgery or RT } \\
+\mathrm{CT} \text {, then } \\
\text { peripheral- } \\
\text { blood-stem-cell } \\
\text { transplant }\end{array}$ & $\begin{array}{l}\text { MOS- } 42.2 \\
\text { mths }\end{array}$ & $\begin{array}{l}\text { Combined } \\
\text { modality therapy } \\
\text { safe in OMBC; } \\
\text { promising relapse- } \\
\text { free survival }\end{array}$ \\
\hline $\begin{array}{l}\text { Milano, } \\
2009[70]\end{array}$ & 3iii / A & $\mathrm{P}$ & 40 & $\leq 5$ met lesions & $\begin{array}{l}\text { Curative-intent } \\
\text { SBRT }\end{array}$ & $\begin{array}{l}\text { 4yr OS- } \\
59 \% ; \text { MOS- } \\
\text { NR }\end{array}$ & $\begin{array}{l}\text { SBRT may yield } \\
\text { prolonged survival } \\
+ \text { perhaps cure in } \\
\text { select OMBC }\end{array}$ \\
\hline $\begin{array}{l}\text { Mimoto, } \\
2014[72]\end{array}$ & 3iii /A & $\mathrm{R}$ & 14 & $\begin{array}{l}1-2 \text { organs with } \\
\text { met lesions, } \leq 5 \\
\text { lesions/organ, } \leq 5 \mathrm{~cm} \\
\text { lesion diameter }\end{array}$ & Surgery & $\begin{array}{l}10 y r \text { OS- } \\
59.2 \%, 20 y r \\
\text { OS-34.1\%; } \\
\text { CD44+/ } \\
\text { CD24-/ } \\
\text { low tumor } \\
\text { cells in 9\% } \\
\text { OMBC } \\
\text { versus } 73 \% \\
\text { non-OMBC }\end{array}$ & $\begin{array}{l}\text { In OMBC, low } \\
\text { levels of cancer- } \\
\text { initiating cells may } \\
\text { be associated with } \\
\text { better prognosis }\end{array}$ \\
\hline $\begin{array}{l}\text { Vander } \\
\text { Walde, } \\
2012[73]\end{array}$ & 3iii /A & $\mathrm{R}$ & 12 & $\leq 3$ sites & $\begin{array}{l}\mathrm{CT}, \text { then } \\
\text { peripheral stem } \\
\text { cell rescue }\end{array}$ & $\begin{array}{l}3-y r \text { OS- } \\
73 \%\end{array}$ & Therapy was safe \\
\hline $\begin{array}{l}\text { Nieto, } 2002 \\
{[75]}\end{array}$ & 3iii /A & $\mathrm{R}$ & 60 & $\begin{array}{l}\text { Low tumor burden, } \\
\text { w met lesion could } \\
\text { be either excised en } \\
\text { bloc before HDC, } \\
\text { or encompassed w } \\
\text { a single RT field w } \\
\text { curative intent. }\end{array}$ & CT & $\begin{array}{l}\text { MOS- } 80 \\
\text { mths; } 5-y r \\
\text { OS } 62 \%\end{array}$ & $\begin{array}{l}\text { Possibly re- } \\
\text { evaluate current } \\
\text { tenet that early } \\
\text { detection MBC is } \\
\text { of no benefit }\end{array}$ \\
\hline $\begin{array}{l}\text { Bourgier, } \\
2010[74]\end{array}$ & $3 \mathrm{iii} / \mathrm{D}$ & $\mathrm{R}$ & 159 & 1 met site & $\begin{array}{l}\text { RT versus RT + } \\
\text { surgery }\end{array}$ & $\begin{array}{l}\text { 3yr OS- } \\
\text { RT- } 39 \% \\
\text { versus } \mathrm{RT}+ \\
\text { surg-57\%; } \\
\text { equivalent } \\
\text { when } \\
\text { adjusted for } \\
\text { prognostic } \\
\text { factors }\end{array}$ & $\begin{array}{l}\text { In sub-analysis, } \\
\text { OMBC had better } \\
\text { metastatic PFS } \\
\text { as compared to } \\
\text { patients with }>1 \\
\text { met site }\end{array}$ \\
\hline
\end{tabular}

Abbreviations: $\operatorname{Met}(\mathrm{s})=$ metastasis (es); $\mathrm{CT}=$ chemotherapy; $\mathrm{yr}=\mathrm{year}$; $\mathrm{OS}=$ overall survival; OMBC $=$ oligometastatic breast cancer; $\mathrm{MBC}=$ metastatic breast cancer; $\mathrm{RT}=$ radiation therapy; $\mathrm{MOS}=$ median overall survival; $\mathrm{NR}=$ not reached; mths=months; SBRT=stereotactic body radiation therapy; HDC=high dose chemotherapy; PFS=progression free survival 
Table 5: Oligometastatic lung cancer

\begin{tabular}{|c|c|c|c|c|c|c|c|}
\hline $\begin{array}{l}\text { 1st Author, Year } \\
\text { [Ref] }\end{array}$ & $\begin{array}{c}\text { Strength of } \\
\text { evidence- } \\
\text { based on } \\
\text { study design / } \\
\text { endpoint }\end{array}$ & $\begin{array}{l}\text { Prospective } \\
\text { (P) or } \\
\text { Retrospective } \\
\text { (R) }\end{array}$ & $\begin{array}{l}\text { Sample } \\
\text { size }\end{array}$ & $\begin{array}{l}\text { Definition- } \\
\text { Oligometastases }\end{array}$ & Therapy & Endpoint & Conclusion \\
\hline $\begin{array}{l}\text { DeRuysscher, } \\
2012 \text { [81] }\end{array}$ & $2 / \mathrm{A}$ & $\mathrm{P}$ & 39 & $\begin{array}{l}<5 \text { synchronous } \\
\text { mets }\end{array}$ & Local trt to mets & $\begin{array}{l}\text { MOS-13.5 } \\
\text { mths. } 3 y r \\
\text { OS- } 17.5 \%\end{array}$ & $\begin{array}{l}\text { Subgroup with } \\
\text { synchronous OM } \\
\text { may benefit from } \\
\text { radical trt }\end{array}$ \\
\hline Collen, 2014 [80] & $2 / \mathrm{A}$ & $\mathrm{P}$ & 26 & $\leq 5$ met lesions & $\begin{array}{l}\text { SBRT to primary } \\
\text { and all mets }\end{array}$ & $\begin{array}{l}\text { MOS-23 } \\
\text { mths. } 1 \mathrm{yr} \\
\text { OS- } 67 \%\end{array}$ & $\begin{array}{l}\text { SBRT acceptable } \\
\text { option and results } \\
\text { in acceptable PFS }\end{array}$ \\
\hline Khan, 2006 [83] & $3 \mathrm{i} / \mathrm{A}$ & $\mathrm{R}$ & 23 & 1-2 sites & $\begin{array}{l}\mathrm{CT}+\text { local- } \\
\text { regional therapy }\end{array}$ & $\begin{array}{l}\text { MOS- } 20 \\
\text { mths }\end{array}$ & $\begin{array}{l}\text { Subset of pats } \\
\text { may benefit } \\
\text { from aggressive } \\
\text { local, regional, } \\
\text { and systemic } \\
\text { treatment }\end{array}$ \\
\hline Nieder, 2014 [82] & $3 \mathrm{i} / \mathrm{A}$ & $\mathrm{R}$ & 23 & $\begin{array}{l}\text { maximum of } 3 \\
\text { metastases to } 1 \\
\text { organ }\end{array}$ & $\begin{array}{l}\text { 'Active therapy', } \\
\text { irrespective of } \\
\text { specific treatment } \\
\text { received }\end{array}$ & $\begin{array}{l}\text { MOS- } 11.7 \\
\text { mths for } \\
\text { OM and } 5.6 \\
\text { mths for } \\
\text { advanced } \\
\text { mets }\end{array}$ & $\begin{array}{l}\text { Prospective } \\
\text { studies for this } \\
\text { population are } \\
\text { warranted }\end{array}$ \\
\hline Guerra 2012 [85] & 3ii/A & $\mathrm{R}$ & 78 & $\begin{array}{l}<5 \text { mets at } \\
\text { diagnosis }\end{array}$ & $\begin{array}{l}\text { Definitive CRT to } \\
\text { primary + mets }\end{array}$ & $\begin{array}{l}3 y r \text { OS- } \\
25 \%\end{array}$ & $\begin{array}{l}\text { Tumor volume, } \\
\text { KPS, + at least } \\
63 \text { Gy to primary } \\
\text { tumor are } \\
\text { associated with } \\
\text { improved OS in } \\
\text { OM NSCLC }\end{array}$ \\
\hline $\begin{array}{l}\text { Ashworth, } 2014 \\
\text { [84] }\end{array}$ & $3 \mathrm{ii} / \mathrm{A}$ & $\mathrm{R}$ & 757 & $\begin{array}{l}\text { Hx of curative trt } \\
\text { to primary and w } \\
1-5 \text { mets treated } \\
\text { w surgery, RT or } \\
\text { XRT }\end{array}$ & $\begin{array}{l}\text { Controlled } \\
\text { primary tumor } \\
\text { and locally } \\
\text { ablative } \\
\text { treatments to all } \\
\text { mets }\end{array}$ & $\begin{array}{l}\text { MOS-26 } \\
\text { mths, } 5 y r \\
\text { OS- } 29.4 \%\end{array}$ & $\begin{array}{l}\text { Significant OS } \\
\text { differences in } \\
\text { OM according to } \\
\text { type of metastatic } \\
\text { presentation and } \\
\text { N status }\end{array}$ \\
\hline $\begin{array}{l}\text { Collaud, } 2012 \\
{[86]}\end{array}$ & $3 \mathrm{iii} / \mathrm{A}$ & $\mathrm{R}$ & 29 & $\begin{array}{l}\text { Synchronous } \\
\text { single organ met }\end{array}$ & $\begin{array}{l}\text { Lung resection } \\
\text { and local trt to } \\
\text { mets }\end{array}$ & $\begin{array}{l}1 \mathrm{yr} \text { OS- } \\
65 \%, 5 \mathrm{yr} \\
\text { OS- } 36 \% ; \\
\text { MOS- } 20.5 \\
\text { mths }\end{array}$ & $\begin{array}{l}\text { Multimodality trt } \\
\text { including lung } \\
\text { resection should } \\
\text { be considered in } \\
\text { select pats }\end{array}$ \\
\hline $\begin{array}{l}\text { Congedo, } 2012 \\
{[87]}\end{array}$ & $3 \mathrm{iii} / \mathrm{A}$ & $\mathrm{R}$ & 53 & $\begin{array}{l}\text { Resected primary } \\
\text { with } 1-2 \text { met } \\
\text { lesions considered } \\
\text { to be resectable }\end{array}$ & $\begin{array}{l}\text { Trt with curative } \\
\text { intent }\end{array}$ & $\begin{array}{l}5 y r \text { OS- } \\
24 \% \\
\text { MOS- } 19 \\
\text { mths }\end{array}$ & $\begin{array}{l}\text { Surgical trt for } \\
\text { selected patients } \\
\text { is feasible and } \\
\text { safe }\end{array}$ \\
\hline $\begin{array}{l}\text { Hasselle } 2012 \\
{[88]}\end{array}$ & 3iii/A & $\mathrm{R}$ & 25 & $\leq 5$ mets & $\begin{array}{l}\text { Hypofractionated } \\
\text { image-guided RT } \\
\text { (HIGRT) }\end{array}$ & $\begin{array}{l}\text { MOS- } 22.7 \\
\text { mths; } \\
18 \mathrm{mth} \text { OS- } \\
52.9 \%\end{array}$ & $\begin{array}{l}\text { HIGRT for OM } \\
\text { NSCLC provides } \\
\text { durable control in } \\
\leq 2 \text { lesions }\end{array}$ \\
\hline
\end{tabular}

(Continued) 


\begin{tabular}{|c|c|c|c|c|c|c|c|}
\hline $\begin{array}{l}\text { 1st Author, Year } \\
\text { [Ref] }\end{array}$ & $\begin{array}{c}\text { Strength of } \\
\text { evidence- } \\
\text { based on } \\
\text { study design / } \\
\text { endpoint }\end{array}$ & $\begin{array}{l}\text { Prospective } \\
\text { (P) or } \\
\text { Retrospective } \\
\text { (R) }\end{array}$ & $\begin{array}{l}\text { Sample } \\
\text { size }\end{array}$ & $\begin{array}{l}\text { Definition- } \\
\text { Oligometastases }\end{array}$ & Therapy & Endpoint & Conclusion \\
\hline $\begin{array}{l}\text { Ashworth, } 2013 \\
\text { [76] }\end{array}$ & 3iii /A & $\mathrm{R}$ & 2176 & $1-5$ mets & $\begin{array}{l}\text { Surgery, SART or } \\
\text { SRS }\end{array}$ & $\begin{array}{l}5 \text { yr OS- } \\
8.3-86 \%, \\
\text { MOS- } \\
\text { range- 5.9- } \\
52 \text { mths }\end{array}$ & $\begin{array}{l}\text { Survival times } \\
\text { for OM were } \\
\text { highly variable, } \\
\text { however long- } \\
\text { term survivors do } \\
\text { exist. }\end{array}$ \\
\hline $\begin{array}{l}\text { Griffioen, } 2013 \\
\text { [89] }\end{array}$ & 3iii /A & $\mathrm{R}$ & 61 & $\begin{array}{l}1-3 \text { synchronous } \\
\text { mets }\end{array}$ & $\begin{array}{l}\text { Radical trt } \\
\text { (Surgery or RT) to } \\
\text { primary and mets }\end{array}$ & $\begin{array}{l}\text { MOS- } 13.5 \\
\text { mths; } 2 \mathrm{yr} \\
\text { OS- } 38 \%\end{array}$ & $\begin{array}{l}\text { Radical trt to } \\
\text { selected pats } \\
\text { can result in } \\
\text { favorable } 2 \mathrm{yr} \\
\text { survival }\end{array}$ \\
\hline Yano 2013 [90] & 3iii/Diii & $\mathrm{R}$ & 13 & $\begin{array}{l}\text { Completely } \\
\text { resected NSCLC, } \\
\text { with post-op } \\
\text { recurrence, } \\
\text { excluding } \\
\text { secondary lung } \\
\text { site. 1-3 distant } \\
\text { mets, not brain } \\
\text { only }\end{array}$ & $\begin{array}{l}\text { Resection or RT } \\
\text { of mets versus CT } \\
\text { of mets }\end{array}$ & $\begin{array}{l}\text { Median } \\
\text { PFS } \\
\text { resection/ } \\
\text { RT -20 } \\
\text { mths; } \\
\text { Median } \\
\text { PFS for CT } \\
\text { was } 5 \text { and } \\
15 \text { mths, } \\
\text { respectively }\end{array}$ & $\begin{array}{l}\text { Local therapy is } \\
\text { a choice for } 1 \mathrm{st} \\
\text { line treatment } \\
\text { in post-op OM } \\
\text { recurrence }\end{array}$ \\
\hline Yu 2013 [91] & 3iii/A & $\mathrm{R}$ & 18 & $\begin{array}{l}\text { EGFR-mutant } \\
\text { lung cancer } \\
\text { previously treated } \\
\text { with erlotinib or } \\
\text { gefitinib, then } \\
\text { progression } \\
\text { on EGFR TKI } \\
\text { therapy, }(<5 \text { sites } \\
\text { disease) }\end{array}$ & $\begin{array}{l}\text { RT, RFA, or } \\
\text { resection of a site } \\
\text { of progressive } \\
\text { disease }\end{array}$ & $\begin{array}{l}\text { MOS } \\
\text { from local } \\
\text { therapy was } \\
41 \text { mths }\end{array}$ & $\begin{array}{l}\text { EGFR-mutant } \\
\text { lung cancers } \\
\text { q acquired } \\
\text { resistance to } \\
\text { EGFR TKI } \\
\text { therapy are } \\
\text { amenable to } \\
\text { local therapy to } \\
\text { treat OM disease } \\
\text { when used in } \\
\text { conjunction with } \\
\text { continued EGFR } \\
\text { inhibition }\end{array}$ \\
\hline Endo, 2014 [98] & 3iii/A & $\mathrm{P}$ & 20 & $\begin{array}{l}\text { single-organ met, } \\
\text { or single-organ } \\
\text { metachronous } \\
\text { met s/p resect } \\
\text { path } \mathrm{T} 1-2 \mathrm{~N} 0-1 \\
\text { lung cancer }\end{array}$ & $\begin{array}{l}\text { Resection primary } \\
\text { tumor and mets }\end{array}$ & $\begin{array}{l}5 y r \text { OS- } \\
44.7 \%\end{array}$ & $\begin{array}{l}\text { Resection of } \\
\text { primary tumor } \\
\text { and mets had } \\
\text { outcomes } \\
\text { comparable to } \\
\text { stage II patients }\end{array}$ \\
\hline Gray, 2014 [92] & $3 \mathrm{iii} / \mathrm{A}$ & $\mathrm{R}$ & 66 & $\begin{array}{l}1-4 \text { synchronous } \\
\text { brain mets }\end{array}$ & $\begin{array}{l}\text { Aggressive } \\
\text { thoracic therapy } \\
\text { (ATT) Surgery } \\
\text { or CRT versus } \\
\text { no-ATT }\end{array}$ & $\begin{array}{l}\text { MOS-26.4 } \\
\text { mths for } \\
\text { ATT versus } \\
10.5 \text { mths } \\
\text { no-ATT }\end{array}$ & $\begin{array}{l}\text { Aggressive } \\
\text { management of } \\
\text { thoracic disease } \\
\text { in OM NSCLC } \\
\text { associated } \\
\text { with improved } \\
\text { survival }\end{array}$ \\
\hline $\begin{array}{l}\text { Cheufou, } 2014 \\
\text { [93] }\end{array}$ & $3 \mathrm{iii} / \mathrm{A}$ & $\mathrm{R}$ & 37 & $\begin{array}{l}\text { Synchronous } \\
\text { single brain met }\end{array}$ & $\begin{array}{l}\text { Resection cerebral } \\
\text { mets and primary } \\
\text { tumor }\end{array}$ & $\begin{array}{l}2 y r \text { OS- } \\
24 \%\end{array}$ & $\begin{array}{l}\text { No increased risk } \\
\text { of complication } \\
\text { or mortality; } \\
\text { median survival } \\
\text { encouraging }\end{array}$ \\
\hline
\end{tabular}

(Continued) 


\begin{tabular}{|c|c|c|c|c|c|c|c|}
\hline $\begin{array}{l}\text { 1st Author, Year } \\
\text { [Ref] }\end{array}$ & $\begin{array}{c}\text { Strength of } \\
\text { evidence- } \\
\text { based on } \\
\text { study design / } \\
\text { endpoint }\end{array}$ & $\begin{array}{l}\text { Prospective } \\
\text { (P) or } \\
\text { Retrospective } \\
\text { (R) }\end{array}$ & $\begin{array}{l}\text { Sample } \\
\text { size }\end{array}$ & $\begin{array}{l}\text { Definition- } \\
\text { Oligometastases }\end{array}$ & Therapy & Endpoint & Conclusion \\
\hline Parikh, 2014 [94] & 3 iii /A & $\mathrm{R}$ & 186 & $\begin{array}{l}\leq 5 \text { synchronous } \\
\text { distant met } \\
\text { lesions }\end{array}$ & $\begin{array}{l}\text { Definitive primary } \\
\text { therapy }\end{array}$ & $\begin{array}{l}\text { MOS-17 } \\
\text { mths for } \\
\text { OM versus } \\
14 \text { mths for } \\
\text { advanced } \\
\text { disease; } \\
\text { Among } \\
\text { OM, MOS- } \\
19 \text { mths for } \\
\text { definitive } \\
\text { therapy } \\
\text { versus } 16 \\
\text { mths for no } \\
\text { definitive } \\
\text { therapy }\end{array}$ & $\begin{array}{l}\text { Definitive therapy } \\
\text { to primary tumor } \\
\text { may provide } \\
\text { survival benefit }\end{array}$ \\
\hline Sheu, 2014 [95] & 3iii/A & $\mathrm{R}$ & 90 & $\begin{array}{l}\leq 3 \text { synchronous } \\
\text { mets }\end{array}$ & \begin{tabular}{|l|} 
CT, then \\
Surgery or RT \\
before disease \\
progression. Then \\
$+/$-comprehensive \\
local therapy \\
(CLT)
\end{tabular} & $\begin{array}{l}\text { MOS- } 22.3 \\
\text { mths; } 1 \mathrm{yr} \\
\text { OS- } 75 \%\end{array}$ & $\begin{array}{l}\text { CLT associated } \\
\text { with improved } \\
\text { OS and PFS with } \\
\text { matched analysis } \\
\text { using propensity } \\
\text { score's }\end{array}$ \\
\hline Tonnies, 2014 [96] & 3iii/A & $\mathrm{R}$ & 99 & $\begin{array}{l}\text { Solitary } \\
\text { hematogenous } \\
\text { metastasis within } \\
3 \text { mths of primary } \\
\text { resection }\end{array}$ & $\begin{array}{l}\text { Primary NSCLC } \\
\text { curatively } \\
\text { resected; then } \\
\text { metastasectomy }\end{array}$ & $\begin{array}{l}5 y r \text { OS- } \\
38 \%\end{array}$ & $\begin{array}{l}\text { Metastasectomy } \\
\text { for synchronous } \\
\text { OM NSCLC can } \\
\text { be performed in } \\
\text { selected patients }\end{array}$ \\
\hline Ouyang, 2014 [97] & 3iii/A & $\mathrm{R}$ & 95 & Not defined & 3DRT + CT & $\begin{array}{l}3 y r \text { OS- } \\
15.8 \%\end{array}$ & $\begin{array}{l}\text { Radiation dose } \\
\geq 63 \mathrm{~Gy} \text { and } \\
\text { having bone only } \\
\text { mets associated } \\
\text { with better } \\
\text { OS; aggressive } \\
\text { thoracic radiation } \\
\text { may play a role in } \\
\text { improving OS }\end{array}$ \\
\hline
\end{tabular}

Abbreviations: $\operatorname{Met}(\mathrm{s})=$ metastasis(es); Trt=treatment; MOS=median overall survival; OM=oligometastases; $\mathrm{SBRT}=$ stereotactic body radiation therapy; $\mathrm{mths}=$ months; $\mathrm{PFS}=$ progression free survival; $\mathrm{CT}=$ chemotherapy; $\mathrm{CRT}=$ chemoradiation therapy; SART=Stereotactic ablative radiation therapy; KPS= Karnofsky performance status; $\mathrm{OS}=$ overall survival; NSCLC $=$ non-small cell lung cancer; $\mathrm{Hx}=$ history; $\mathrm{RT}=$ radiation therapy; XRT=external radiation therapy; $\mathrm{N}=$ node; $\mathrm{EGFR}=$ epidermal growth factor receptor; $\mathrm{TKI}=$ tyrosine kinase inhibitor; $\mathrm{RFA}=$ radio-frequency ablation

studies conducted. Essner et al. was a population-based consecutive case series (Level 3i), which included a large sample size $(n=877)$, and determined factors prognostic for increased survival [99]. The second study was a nonconsecutive case series (Level 3iii) with a more moderate sample size [100]. The two studies differed in their therapies, definitions of oligometastases, and interval endpoint of OS. Although OS was reported in both studies (Level A), it was reported in different intervals; 2-year (19.7-47\%) [100] and 5-year (17-30\%) [99].

\section{Colorectal cancer}

Colorectal cancer is the fourth most common cancer diagnosis in the world (around 1.2 million diagnoses each year), and accounts for the second highest number of deaths [37]. Nearly one-fourth of patients with newly diagnosed colorectal cancer (CRC) will present with synchronous liver metastases [101, 102]. Hepatic resection is considered a standard treatment option for metastatic CRC and can result in 10-year survival rates of $20-26 \%$, and potential cure [103-109]. 
Table 6: Oligometastatic melanoma

\begin{tabular}{|c|c|c|c|c|c|c|c|}
\hline $\begin{array}{l}\text { 1st Author, } \\
\text { Year [Ref]b }\end{array}$ & $\begin{array}{l}\text { Strength of } \\
\text { evidence- } \\
\text { based } \\
\text { on study } \\
\text { design / } \\
\text { endpoint }\end{array}$ & $\begin{array}{c}\text { Pros } \\
\text { pective } \\
\text { (P) or } \\
\text { Retros } \\
\text { pective } \\
\text { (R) }\end{array}$ & $\begin{array}{l}\text { Sample } \\
\text { size }\end{array}$ & $\begin{array}{l}\text { Definition- } \\
\text { Oligo } \\
\text { metastases }\end{array}$ & Therapy & Endpoint & Conclusion \\
\hline $\begin{array}{l}\text { Essner, } \\
2004 \text { [99] }\end{array}$ & $3 \mathrm{i} / \mathrm{A}$ & $\mathrm{R}$ & 877 & $1 \mathrm{met}$ & Curative surgery & $\begin{array}{l}5 y r \text { OS- } 29 \text { mths if } \\
\text { mets } 1 \text { site, } 16 \text { mths if } \\
\text { mets } 2-3 \text { sites, } 14 \text { mths } \\
\text { if met } \geq 4 \text { sites. } 5 y r \\
\text { OS- } 17 \% \text { disease-free } \\
\text { if distant mets in }<36 \\
\text { mths, } 30 \% \text { if }>36 \text { mths }\end{array}$ & $\begin{array}{l}\text { Pats with limited } \\
\text { mets should } \\
\text { be considered } \\
\text { for curative } \\
\text { resection }\end{array}$ \\
\hline $\begin{array}{l}\text { Knisely, } \\
2012 \text { [100] }\end{array}$ & 3iii /A & $\mathrm{R}$ & 77 & $\begin{array}{l}\text { Brain mets } \\
\text { treated with } \\
\text { SRS }\end{array}$ & $\begin{array}{l}\text { SRS to brain } \\
\text { mets, then } 35 \% \\
\text { of group received } \\
\text { ipilimumab }\end{array}$ & $\begin{array}{l}\text { MOS- } 21.3 \text { mths in } \\
\text { ipilimumab group } \\
\text { versus } 4.9 \text { mths } \\
\text { in no-ipilimumab } \\
\text { group. } 2 \text { yr OS- } 47 \% \\
\text { in ipilimumab group } \\
\text { and } 19.7 \% \text { in no- } \\
\text { ipilimumab group }\end{array}$ & $\begin{array}{l}\text { Survival of } \\
\text { patients with } \\
\text { melanoma and } \\
\text { brain mets } \\
\text { managed with } \\
\text { ipilimumab }+ \\
\text { SRS can exceed } \\
\text { expected 4-6 } \\
\text { mths }\end{array}$ \\
\hline
\end{tabular}

Abbreviations: $\operatorname{Met}(\mathrm{s})=$ metastasis(es); $\mathrm{OS}=$ overall survival; $\mathrm{SRS}=$ stereotactic radiosurgery; $\mathrm{MOS}=$ median overall survival

We searched PubMed using the terms 'colorectal cancer oligometastasis', 'colorectal cancer oligometastases', and 'oligometastatic colorectal cancer'; results were as follows, $n=5, n=24$, and $n=28$, respectively. We omitted reviews, studies including mixed primary tumors, studies not focused on oligometastatic colorectal cancer, case reports, perspectives, preclinical reports, and earlier reports if later reports for the same study were available. There were nine studies remaining (Table 7); oligometastasis held 9 different definitions and each study had a different treatment paradigm.

The evidence for treatment of oligometastases in colorectal cancer is weak-to-moderate based on the study designs. There were two prospective, nonrandomized controlled clinical trials (Level 2) studies [104, 110], however both with small sample sizes $(n=24$ and $n=9$, respectively) and one prospective nonconsecutive case series (Level 3iii) study with a moderate sample size $(n=82)$ [111]. Salah et al. conducted a pooled analysis of 8 studies with a large sample size $(n=927)$ and reported 5-yr survival, however it was retrospective and a nonconsecutive case series (Level 3iii) [112]. The remaining five studies were non population-based consecutive case series (Level 3ii) [113, 114] and Level 3 iii [115-117]. Overall survival endpoints (Level A) were reported in all but one study [110], however stronger study designs would have shown whether the survival endpoints were significant. OS was reported at three intervals: 5 -year
(52-58\%) [115], 54.3\% [112], 39\% [114], 38\% [116] and 29\%) [117], 3-year (43\%) [111], and 1-year (78\%) [104] and 53\%) [113].

\section{Sarcoma}

Soft tissue sarcomas are relatively rare with about 10, 000 cases diagnosed each year in the United States. If diagnosed early, the prognosis is excellent, however, in some subtypes (of which there are greater than 50), up to $50 \%$ of patients will develop distant metastases and will have a 5-year survival of below $10 \%$. The median overall survival for patients with advanced and metastatic soft tissue sarcoma is about 12 months [118].

We searched PubMed using the terms 'sarcoma oligometastasis', 'sarcoma oligometastases', and 'oligometastatic sarcoma'; results were as follows, $n=3, n=$ 3 , and $n=13$, respectively. We omitted reviews, studies including mixed primary tumors, studies not focused on oligometastatic sarcoma, and case reports. There were two clinical studies remaining (Table 8); both studies used different definitions of oligometastasis and a different treatment paradigm.

The evidence for oligometastases in sarcoma was weak based on the two retrospective studies available for review. Both studies were nonconsecutive case series (Level 3iii). Falk et al. included a moderate sample size $(n=281)$ and MOS was reported (45.3\%) (Level A) [119]. The second study was small $(n=16)$ and the endpoint was time to progression (17-months) (Level Diii) [120]. 
Table 7: Oligometastatic colorectal cancer

\begin{tabular}{|c|c|c|c|c|c|c|c|}
\hline $\begin{array}{l}\text { 1st } \\
\text { Author, } \\
\text { Year [Ref] }\end{array}$ & $\begin{array}{l}\text { Strength of } \\
\text { evidence- } \\
\text { based } \\
\text { on study } \\
\text { design / } \\
\text { endpoint }\end{array}$ & $\begin{array}{c}\text { Prospective } \\
\text { (P) / } \\
\text { Retrospective } \\
\text { (R) }\end{array}$ & $\begin{array}{l}\text { Sample } \\
\text { size }\end{array}$ & $\begin{array}{l}\text { Definition- } \\
\text { Oligo } \\
\text { metastases }\end{array}$ & Therapy & Endpoint & Conclusion \\
\hline $\begin{array}{l}\text { Engels, } \\
2012 \text { [104] }\end{array}$ & $2 / \mathrm{A}$ & $\mathrm{P}$ & 24 & $\leq 5$ mets & $\begin{array}{l}\text { Resected primary } \\
\text { tumor, and inoperable } \\
\text { mets treated with } \\
\text { helical tomotherapy }\end{array}$ & 1yr OS- $78 \%$ & $\begin{array}{l}\text { Helical tomotherapy } \\
\text { is an attractive for } \\
\text { consolidation of } \\
\text { inoperable OM disease } \\
\text { after effective chemo }\end{array}$ \\
\hline $\begin{array}{l}\text { Dellas, } \\
2012[110]\end{array}$ & $2 / \mathrm{D}$ & $\mathrm{P}$ & 9 & $\begin{array}{l}1-3 \text { mets } \\
\text { or local } \\
\text { recurrence plus } \\
\text { max. } 2 \text { mets }\end{array}$ & $\begin{array}{l}\mathrm{CT}+3 \mathrm{D}-\mathrm{CRT} \text { to all } \\
\text { met lesions }\end{array}$ & $\begin{array}{l}3 / 9 \text { pats } \\
\text { survived } \\
3.5-4.4 \text { yrs; } \\
\text { DLT not } \\
\text { documented }\end{array}$ & $\begin{array}{l}\text { 3D-CRT to mets } \\
\text { feasible in addition to } \\
\text { standard CT }\end{array}$ \\
\hline $\begin{array}{l}\text { Van den } \\
\text { Begin, } \\
2014[113]\end{array}$ & 3ii/A & $\mathrm{R}$ & 47 & $\begin{array}{l}\text { Resected } \\
\text { primary }+\leq 5 \\
\text { mets, liver, } \\
\text { lung, LNs }\end{array}$ & SBRT & $1 \mathrm{yr}$ OS- $53 \%$ & $\begin{array}{l}\text { Nature }+ \text { location } \\
\text { of local recurrences } \\
\text { demonstrated need for } \\
\text { breathing management } \\
\text { and dose }>75 \mathrm{~Gy} \text {. }\end{array}$ \\
\hline $\begin{array}{l}\text { Filippi, } \\
2014 \text { [114] }\end{array}$ & 3ii/A & $\mathrm{R}$ & 40 & $\begin{array}{l}\text { Resected } \\
\text { primary }+1-5 \\
\text { lung mets, max } \\
\text { diameter }<5 \mathrm{~cm}\end{array}$ & $\begin{array}{l}\text { 3D conformational } \\
\text { RT or image guided } \\
\text { volumetric modulated } \\
\text { arc therapy }\end{array}$ & $5 y r$ OS- $39 \%$ & $\begin{array}{l}\text { Suggests stereotactic } \\
\text { ablative RT is safe } \\
+ \text { efficacious in } \\
\text { CRC with lung } \\
\text { oligometastases }\end{array}$ \\
\hline $\begin{array}{l}\text { Kang, } \\
2010 \text { [117] }\end{array}$ & $3 \mathrm{iii} / \mathrm{A}$ & $\mathrm{R}$ & 59 & $\begin{array}{l}1-4 \text { met lesions } \\
\text { confined } \\
\text { to } 1 \text { organ, } \\
\text { largest }<7 \mathrm{~cm} \text {, } \\
\text { progressive } \\
\text { after } \mathrm{CT}\end{array}$ & $\begin{array}{l}\text { Met lesions } \\
\text { progressed after } \\
\text { chemo, then treated } \\
\text { SBRT }\end{array}$ & $5 y r$ OS- $29 \%$ & $\begin{array}{l}\text { Patients generally fare } \\
\text { well after SBRT }\end{array}$ \\
\hline $\begin{array}{l}\text { Salah, } \\
2012 \text { [112] }\end{array}$ & $3 \mathrm{iii} / \mathrm{A}$ & $\mathrm{R}$ & 927 & $\begin{array}{l}\text { Underwent } \\
\text { lung } \\
\text { metastasectomy }\end{array}$ & Lung metastasectomy & \begin{tabular}{|l|}
$5 y r$ OS- \\
$54.3 \%$. \\
Prognostic risk \\
groups good-, \\
intermediate-, \\
and high-, had \\
5 yr OS- $68 \%$, \\
$46 \%, 26 \%$
\end{tabular} & $\begin{array}{l}\text { More studies need to } \\
\text { investigate if surgery } \\
\text { offers advantage over } \\
\text { CT in the poor-risk } \\
\text { group }\end{array}$ \\
\hline $\begin{array}{l}\text { Bae, } 2012 \\
{[116]}\end{array}$ & 3iii/ A & $\mathrm{R}$ & 41 & $\begin{array}{l}\text { Met lesions } \\
\text { confined to } 1 \\
\text { organ }\end{array}$ & 3 fractions SBRT & $5 y r$ OS- $38 \%$ & $\begin{array}{l}\text { SBRT results } \\
\text { comparable with } \\
\text { Surgery }\end{array}$ \\
\hline $\begin{array}{l}\text { Salah, } \\
2013 \text { [115] }\end{array}$ & 3iii /A & $\mathrm{R}$ & 148 & $\begin{array}{l}\text { Repeat } \\
\text { resection of } \\
\text { pulmonary } \\
\text { mets }\end{array}$ & $\begin{array}{l}\text { Repeat resection of } \\
\text { lung met }\end{array}$ & \begin{tabular}{|l|}
5 yr OS- $52 \%$ \\
for 1 lung met \\
resection, $58 \%$ \\
2 lung met \\
resection. $>2$ \\
lung mets $+\geq 3$ \\
cm risk factors \\
for decreased \\
survival
\end{tabular} & $\begin{array}{l}\text { In selected patients, } \\
\text { repeated pulmonary } \\
\text { resection offers good } \\
\text { survival outcome }\end{array}$ \\
\hline $\begin{array}{l}\text { Comito, } \\
2014[111]\end{array}$ & 3iii/A & $\mathrm{P}$ & 82 & $\begin{array}{l}1-3 \text { inoperable } \\
\text { mets in } 1 \text { organ } \\
\text { (Liver or lung) }\end{array}$ & SBRT & 3yr OS- $43 \%$ & $\begin{array}{l}\text { SBRT safe }+ \text { feasible } \\
\text { treatment for mets not } \\
\text { amenable to resection }\end{array}$ \\
\hline
\end{tabular}

Abbreviations: $\operatorname{Met}(\mathrm{s})=$ metastasis(es); $\mathrm{OS}=$ overall survival; $\mathrm{CT}=$ chemotherapy; $\mathrm{CRT}=$ chemoradiation therapy;

$\mathrm{DLT}=$ dose-limiting toxicity; $\mathrm{SBRT}=$ stereotactic body radiation therapy; LNs=lymph nodes; RT=radiation therapy;

$\mathrm{CRC}=$ colorectal cancer 
Table 8: Oligometastatic sarcoma

\begin{tabular}{|c|c|c|c|c|c|c|c|}
\hline $\begin{array}{l}\text { 1st Author, } \\
\text { Year [Ref] }\end{array}$ & $\begin{array}{c}\text { Strength of } \\
\text { evidence- } \\
\text { based on } \\
\text { study design / } \\
\text { endpoint }\end{array}$ & $\begin{array}{l}\text { Prospective } \\
\text { (P) or } \\
\text { Retrospective } \\
\text { (R) }\end{array}$ & $\begin{array}{c}\text { Sample } \\
\text { size }\end{array}$ & $\begin{array}{l}\text { Definition- } \\
\text { Oligometastases }\end{array}$ & Therapy & Endpoint & Conclusion \\
\hline $\begin{array}{l}\text { Falk, } 2014 \\
{[119]}\end{array}$ & $3 \mathrm{ii} / \mathrm{A}$ & $\mathrm{R}$ & 281 & $\begin{array}{l}1-5 \text { lesions, any } \\
\text { site }\end{array}$ & $\begin{array}{l}164 / 281 \text { pats received } \\
\text { local treatment (surgery, } \\
\text { radiofrequency ablation, } \\
\text { or RT }\end{array}$ & $\begin{array}{l}\text { MOS- was } \\
45.3 \text { for local } \\
\text { treatment } \\
\text { group and } \\
\text { was12.6 mths } \\
\text { for non-local } \\
\text { treatment } \\
\text { group }\end{array}$ & $\begin{array}{l}\text { Local ablative } \\
\text { treatment seemed } \\
\text { to improve } \\
\text { OS. Surgery } \\
\text { yielded most } \\
\text { relevant results, } \\
\text { alternative } \\
\text { approaches were } \\
\text { promising }\end{array}$ \\
\hline $\begin{array}{l}\text { Rhomberg, } \\
2008[120]\end{array}$ & 3iii/Diii & $\mathrm{R}$ & 16 & $<7$ distant mets & $\begin{array}{l}\text { Received CT + RT +/- } \\
\text { surgery; }\end{array}$ & $\begin{array}{l}\text { Median } \\
\text { survival until } \\
1 \text { st distant } \\
\text { mets- } 17 \\
\text { mths in OM } \\
\text { sarcoma } \\
\text { versus } 9 \\
\text { months in } \\
\text { control group }\end{array}$ & $\begin{array}{l}\text { Razoxane, } \\
\text { vindesine }+ \text { RT } \\
\text { feasible in early } \\
\text { met soft tissue } \\
\text { sarcoma; inhibits } \\
\text { development of } \\
\text { remote mets in } \\
\text { most patients }\end{array}$ \\
\hline
\end{tabular}

Abbreviations: $\mathrm{RT}=$ radiation therapy; $\mathrm{MOS}=$ median overall survival; $\mathrm{OS}=$ overall survival; mets=metastases;

$\mathrm{CT}=$ chemotherapy; $\mathrm{OM}=$ oligometastases

\section{Renal cell carcinoma}

Approximately $25-30 \%$ of patients diagnosed with renal cell carcinoma have metastatic disease at initial presentation. Approximately $1 / 3$ with clinically localized primary tumor at diagnosis will eventually develop metastatic disease [121]. Historically patients with metastatic RCC (mRCC) have a poor prognosis, with 5 -yr survival rates of $\leq 10 \%$, however prolonged survival has been noted in those with solitary or oligometastatic disease amenable to resection. RCC is often considered resistant to cytotoxic chemoconventional RT and cytokinebased immunotherapy. The lung is the most common site of metastases in $\mathrm{mRCC}$; the second most common is bone. SABR is being suggested as a potential new therapeutic option.

The standard of care in mRCC is systemic therapy; however, in patients with solitary or limited metastases, aggressive local therapies may potentially prolong survival. The literature suggests a survival benefit with surgical metastasectomy, with a reported 5-year survival as high as $45 \%$ in those who achieve complete resection. [55]

We searched PubMed using the terms 'renal cell carcinoma oligometastasis', 'renal cell carcinoma oligometastases', and 'oligometastatic renal cell carcinoma'; results were as follows, $n=1, n=9$, and $n=3$, respectively. We omitted reviews, studies including mixed primary tumors, and studies not focused on oligometastatic renal cell carcinoma. There were three clinical studies remaining (Table 9); each study used a different definition of oligometastases and a different treatment paradigm.

The evidence for treatment of oligometastases in renal cell carcinoma is weak based on the study designs. There were three retrospective studies with smaller sample sizes; a non population-based consecutive case series (Level 3ii) [122] and two nonconsecutive case series (Level 3iii) [123, 124]. Overall survival endpoints (Level A) were reported in at three intervals: 5-year (27\%) [124], 2-year (86\%) [122], and 1-year (83.9\%) [123].

Additional searching through the bibliographies of other selected papers allowed us to retrieve two clinical studies that investigated the effects of removal of the primary tumor in patients with metastatic renal cell carcinoma (mRCC) (Table 9) $[125,126]$. The evidence for removal of the primary tumor in $\mathrm{mRCC}$ was strong; both studies were nonblinded randomized controlled clinical trials (Level 1ii), had moderate sample sizes, and reported median OS (17 versus 7 months, [125] and 11.1 versus 8.1 months [126]) (Level A). The therapies given in both studies were similar: nephrectomy + interferon versus interferon only [125] and nephrectomy was followed by interferon versus interferon only [126]. The evidence for oligometastases in RCC was not assessed in these studies. However, they were important studies owing to strong design, with comparable treatments and endpoints, and most importantly they demonstrated that removal of the primary tumor in metastatic disease increased survival. 
Table 9: Oligometastatic Renal Cell Carcinoma

\begin{tabular}{|c|c|c|c|c|c|c|c|}
\hline $\begin{array}{l}\text { 1st Author, } \\
\text { Year [Ref] }\end{array}$ & $\begin{array}{l}\text { Strength of } \\
\text { evidence- } \\
\text { based } \\
\text { on study } \\
\text { design / } \\
\text { endpoints }\end{array}$ & $\begin{array}{l}\text { Prospective } \\
\text { (P) or } \\
\text { Retrospective } \\
\text { (R) }\end{array}$ & $\begin{array}{l}\text { Sample } \\
\text { size }\end{array}$ & $\begin{array}{l}\text { Definition- } \\
\text { Oligometastases }\end{array}$ & Therapies & Endpoints & Conclusion \\
\hline $\begin{array}{l}\text { Mickisch, } 2001 \\
{[125]}\end{array}$ & $1 \mathrm{ii} / \mathrm{A}$ & $\mathrm{P}$ & 85 & $\begin{array}{l}\text { N/A- patients } \\
\text { identified as having } \\
\text { metastatic RCC }\end{array}$ & $\begin{array}{l}\text { Surgery + } \\
\text { interferon } \\
\text { OR } \\
\text { interferon } \\
\text { only }\end{array}$ & $\begin{array}{l}\text { TTP (5 versus } \\
3 \mathrm{mths})+ \\
\text { MOS }(17 \\
\text { versus } 7 \mathrm{mths}) \\
\text { in Surgery } \\
+ \text { interferon } \\
\text { versus } \\
\text { interferon } \\
\text { only }\end{array}$ & $\begin{array}{l}\text { Radical nephrectomy } \\
\text { before interferon- } \\
\text { based immunotherapy } \\
\text { may delay TTP and } \\
\text { improve survival in } \\
\text { mRCC }\end{array}$ \\
\hline $\begin{array}{l}\text { Flanigan, } 2001 \\
{[126]}\end{array}$ & $1 \mathrm{ii} / \mathrm{A}$ & $\mathrm{P}$ & 241 & $\begin{array}{l}\text { N/A- patients } \\
\text { identified as having } \\
\text { metastatic RCC }\end{array}$ & $\begin{array}{l}\text { Surgery } \\
\text { followed by } \\
\text { interferon } \\
\text { OR } \\
\text { interferon } \\
\text { alone }\end{array}$ & \begin{tabular}{|l} 
Surgery \\
followed by \\
interferon \\
MOS- 11.1 \\
mths versus \\
interferon \\
alone MOS- \\
8.1 mths
\end{tabular} & $\begin{array}{l}\text { Nephrectomy } \\
\text { followed by interferon } \\
\text { had longer survival }\end{array}$ \\
\hline $\begin{array}{l}\text { Bang, } 2012 \\
{[124]}\end{array}$ & 3iii/A & $\mathrm{R}$ & 27 & $\begin{array}{l}\text { Localized soft } \\
\text { tissue mass }<7 \mathrm{~cm} \\
+\leq 5 \text { lesions in } 1 \\
\text { organ }\end{array}$ & Cryoablation & $5 y r$ OS- $27 \%$ & $\begin{array}{l}\text { Multisite cryoablation } \\
\text { of OM RCC } \\
\text { associated with low } \\
\text { morbidity and low } \\
\text { recurrence with } \\
\text { apparent increased OS }\end{array}$ \\
\hline $\begin{array}{l}\text { Ranck, } 2013 \\
{[122]}\end{array}$ & $3 \mathrm{ii} / \mathrm{A}$ & $\mathrm{R}$ & 18 & $\begin{array}{l}\text { Limited metastatic } \\
\text { disease }\end{array}$ & $\begin{array}{l}\text { SBRT: } 3 \\
\text { fractions or } \\
10 \text { fractions }\end{array}$ & $2 \mathrm{yr}$ OS- $85 \%$ & $\begin{array}{l}\text { SBRT produces } \\
\text { promising lesion } \\
\text { control with minimal } \\
\text { toxicity }\end{array}$ \\
\hline $\begin{array}{l}\text { Thibault, } 2014 \\
{[123]}\end{array}$ & 3iii/A & $\mathrm{R}$ & 13 & $<5$ spinal mets & SBRT & $\begin{array}{l}1 \text { yr OS- } \\
83.9 \% \text { in OM } \\
\text { RCC }(n=13) \\
\text { versus } 52.5 \% \\
\text { in non-OM } \\
\text { RCC }(n=24)\end{array}$ & $\begin{array}{l}\text { Multivariate analysis } \\
\text { identified OM RCC } \\
\text { as a prognostic factor } \\
\text { for survival. OM RCC } \\
\text { may benefit the most } \\
\text { from aggressive local } \\
\text { therapy }\end{array}$ \\
\hline
\end{tabular}

Abbreviations: $\mathrm{TTP}=$ time to progression; $\mathrm{mRCC}=$ metastatic renal cell carcinoma; $\mathrm{MOS}=$ median overall survival; $\mathrm{OM}=$ oligometastatic; $\mathrm{OS}=$ overall survival; $\mathrm{SBRT}=$ stereotactic body radiation therapy; mets= metastases

\section{Prostate cancer}

There is a body of evidence, contrary to historical clinical practice, that treating men with metastatic prostate cancer to the lymph nodes at the time of diagnosis with surgery or radiation therapy results in increased longterm survival (Table 10), [127-131] and Table 11 [101, 132]. Historically, the ideal patient to be cured by radical prostatectomy (RP) was one with organ-confined cancer. At that time, the morbidity of RP was substantial; therefore the surgery was generally only offered to those where the probability of cure was high. More recent data, however, show that RP may provide a survival benefit- albeit not a cure - to men with metastatic prostate cancer. Engel et al. [101] conducted a retrospective study $(n=938)$ and found that in men with prostate cancer and positive lymph nodes, treated with $+/-\mathrm{RP}$, that the 5- and 10-yr survival rates and the prostate cancer specific survival rates, were better in men who had undergone RP. Cadeddu et al [132] conducted a retrospective study $(n=38)$ and found that in men with prostate cancer and positive lymph nodes, treated with LND +/- RP, that the 5- and 10-yr prostate cancer specific survival was better in men who had undergone LND + RP. Ost et al (2014) conducted a systemic review of the literature of metastasis-directed therapy of regional and distant recurrences after curative treatment for $\mathrm{PCa}$ (prostate cancer). They found that 
Table 10: Prostate cancer (staged T1c-T3b [198])

\begin{tabular}{|c|c|c|c|c|c|c|c|}
\hline $\begin{array}{l}\text { 1st Author, } \\
\text { Year [Ref] }\end{array}$ & $\begin{array}{l}\text { Study } \\
\text { population }\end{array}$ & $\begin{array}{l}\text { Strength of } \\
\text { evidence- } \\
\text { based on } \\
\text { study design } \\
\text { / endpoint }\end{array}$ & $\begin{array}{l}\text { Prospective (P) } \\
\text { / Retrospective } \\
\text { (R) }\end{array}$ & $\begin{array}{l}\text { Sample } \\
\text { size }\end{array}$ & Therapy & Endpoints & Conclusion \\
\hline $\begin{array}{l}\text { Widmark } \\
\text { (2009) [127] }\end{array}$ & $\begin{array}{l}\text { Locally } \\
\text { advanced }\end{array}$ & $1 \mathrm{ii} / \mathrm{A}$ & $\mathrm{P}$ & 875 & $\begin{array}{l}\text { Primary: } \\
\text { HT +/- RT }\end{array}$ & $\begin{array}{l}\text { 10yr PCa } \\
\text { specific } \\
\text { mortality in } \\
\text { ET + RT group } \\
\text { and the ET } \\
\text { alone group } \\
\text { was } 11.9 \% \text { and } \\
23.9 \% .10 y r \\
\text { PSA recurrence } \\
\text { higher in the } \\
\text { ET alone group } \\
(74.7 \% \text { vs. } \\
29.5 \%\end{array}$ & $\begin{array}{l}\text { Addition of RT } \\
\text { to ET, halved 10- } \\
\text { yr PCa-specific } \\
\text { mortality and } \\
\text { decreased overall } \\
\text { mortality in } \\
\text { locally advanced } \\
\text { PCa }\end{array}$ \\
\hline $\begin{array}{l}\text { Vickers } \\
(2012)[128]\end{array}$ & $\mathrm{T} 1-\mathrm{T} 2$ & 1ii /B & $\mathrm{P}$ & 695 & $\begin{array}{l}\text { Primary: } \\
\text { RP }\end{array}$ & $\begin{array}{l}\text { RP beneficial } \\
\text { to Gleason } 8, \\
\text { or Gleason } 7 \text {, } \\
\text { stage } 2\end{array}$ & $\begin{array}{l}\text { Younger men w } \\
\text { more aggressive } \\
\text { disease had } \\
\text { larger reduction } \\
\text { in risk of } \mathrm{PCa} \\
\text { death }\end{array}$ \\
\hline $\begin{array}{l}\text { Zelefsky } \\
(2010) \text { [129] }\end{array}$ & $\begin{array}{l}\text { Clinically } \\
\text { localized: } \\
\text { T1c-T3b }\end{array}$ & $3 \mathrm{iii} / \mathrm{B}$ & $\mathrm{R}$ & 2380 & $\begin{array}{l}\text { Primary: } \\
\text { RP or RT } \\
\text { to prostate }\end{array}$ & $\begin{array}{l}\text { 8-year } \\
\text { probability of } \\
\text { freedom from } \\
\text { met progression } \\
\text { was } 97 \% \text { for } \\
\text { RP and } 93 \% \text { for } \\
\text { EBRT }\end{array}$ & $\begin{array}{l}\text { RP with higher } \\
\text { risk disease, had } \\
\text { lower risk of } \\
\text { met progression } \\
\text { and PCa specific } \\
\text { death }\end{array}$ \\
\hline $\begin{array}{l}\text { Pierorazio } \\
(2013)[130]\end{array}$ & $\begin{array}{l}\text { Up to } \\
\text { clinically } \\
\text { localized }\end{array}$ & 3iii /B & $\mathrm{R}$ & 842 & $\begin{array}{l}\text { Primary } \\
\text { + LNs: } \\
\text { RP+PLND }\end{array}$ & $\begin{array}{l}P S A \geq 20 \text { and } \\
\text { perineural } \\
\text { invasion } \\
\text { at biopsy } \\
\text { increased } \\
\text { likelihood of } \\
\text { unfavorable, } \\
\text { high-grade } \\
\text { disease. }\end{array}$ & $\begin{array}{l}\text { High-Gleason } \\
\text { PCa not } \\
\text { uniformly } \\
\text { associated with } \\
\text { poor outcomes } \\
\text { after RP, but } \\
\text { unfavorable } \\
\text { (pT3b/N1) } \\
\text { disease fared } \\
\text { poorly }\end{array}$ \\
\hline $\begin{array}{l}\text { Shao (2014) } \\
{[131]}\end{array}$ & Localized & $3 \mathrm{iii} / \mathrm{B}$ & $\mathrm{R}$ & 916 & $\begin{array}{l}\text { Primary: } \\
\text { RP or RT }\end{array}$ & $\begin{array}{l}\text { RP had longer } \\
\text { PCaSS as } \\
\text { compared to } \\
\text { primary RT }\end{array}$ & $\begin{array}{l}\text { Results add to } \\
\text { the growing } \\
\text { evidence that } \\
\text { controlling the } \\
\text { primary site may } \\
\text { be important in } \\
\text { patient with met } \\
\text { cancer }\end{array}$ \\
\hline
\end{tabular}

Abbreviations: $\mathrm{HT}=$ hormone therapy; $\mathrm{RT}=$ radiation therapy; $\mathrm{PCa}=$ prostate cancer; $\mathrm{ET}=$ endocrine therapy; $\mathrm{RP}=\mathrm{radical}$ prostatectomy; EBRT=external beam radiation therapy; LNs=lymph nodes; PLND=positive lymph node dissection; $\mathrm{PCaSS}=$ prostate cancer specific survival; met $=$ metastatic 
salvage LND and RT appear to be safe in treatments for OM PCa recurrence. [133] Culp et al. [134] studied the impact of survival of definitive treatment of the prostate in men diagnosed with metastatic PCa. Using the SEER database, he reviewed 8185 men treated with NSR (no surgery no radiation), brachytherapy, or RT. The 5-year OS and disease specific survival (DSS) was significantly higher in men with metastases having undergone RT.

Zapatero et al., reported on a study of men with intermediate and high risk localized prostate cancer $(n=362)$, treated with RT + long term ADT versus $\mathrm{RT}+$ short term ADT, and found that long term ADT was superior in 5-year biochemical disease free survival, metastasis free survival, and OS [135].

The standard of care treatment for metastatic prostate cancer is androgen deprivation therapy (ADT) [136]. Men with newly diagnosed metastatic prostate cancer, entered into the control arm of the Systemic Therapy Multi-Arm Randomized Controlled Trial (STAMPEDE) and treated with $\geq 2$ years of ADT alone, had a 2 -year OS of $72 \%$ [137]. The response rate for primary hormonal therapy for men with metastatic prostate cancer exceeds $80 \%$ and the median duration of response is approximately 18-24 months [138]. In men with prostate cancer with limited metastases, radical prostatectomy may be advantageous in that the primary tumor and its ability to continuously metastasize, to secrete tumor promoting growth factors and immunosuppressive cytokines, and to generate bulk-related morbidity, is removed. Heidenreich et al. recently evaluated survival outcomes following radical prostatectomy (RP) in men with low volume metastatic prostate cancer. RP led to improved progression-free survival, time to castrate resistance and overall survival, as compared to a cohort treated with androgen deprivation therapy alone. [139] Moreover, Abdollah et al. found that in men with $\mathrm{pN} 1$ prostate cancer, treated with RP and extended lymph node dissection, adding adjuvant radiotherapy improved cancer-specific mortality [140].

We searched PubMed using the terms 'prostate cancer oligometastasis', 'prostate cancer oligometastases', and 'oligometastatic prostate cancer'; results were as follows, $n=3, n=19$, and $n=22$, respectively. We omitted reviews, studies including mixed primary tumors, studies not focused on oligometastatic prostate cancer, case reports, editorials/commentaries, studies that we were not able retrieve, and studies with no results reported. There were ten clinical studies remaining (Table 11); oligometastasis was defined differently in each study and there were 7 different treatment paradigms.

The evidence for treatment of oligometastases in prostate cancer is weak based on the study designs being all nonconsecutive case series (Level 3iii) [101, 132, 133, 141-149], although Ost el al. included a large sample (450 men) [133]. Overall survival endpoints

\section{Table 11: Oligometastatic prostate cancer}

\begin{tabular}{|c|c|c|c|c|c|c|c|}
\hline $\begin{array}{l}\text { 1st Author, } \\
\text { Year [Ref] }\end{array}$ & $\begin{array}{l}\text { Strength } \\
\text { of } \\
\text { evidence- } \\
\text { based } \\
\text { on study } \\
\text { design / } \\
\text { endpoints }\end{array}$ & $\begin{array}{l}\text { Prospective } \\
\text { (P) / } \\
\text { Retrospective } \\
\text { (R) }\end{array}$ & $\begin{array}{l}\text { Sample } \\
\text { size }\end{array}$ & $\begin{array}{l}\text { Definition- } \\
\text { Oligometa } \\
\text { stases }\end{array}$ & Therapy & Endpoints & Conclusion \\
\hline $\begin{array}{l}\text { James, } 2014 \\
{[137]}\end{array}$ & $1 \mathrm{ii} / \mathrm{A}$ & $\mathrm{P}$ & 917 & $\begin{array}{l}\mathrm{N} / \mathrm{A}-\text { newly } \\
\text { diagnosed } \mathrm{M} 1\end{array}$ & LT ADT & $\begin{array}{l}\text { FFS- } 11 \text { mths. } 2 y r \\
\text { FFS- } 29 \% \text {. MOS- } 42 \\
\text { mth. } 2 \text { yr OS- } 72 \% .\end{array}$ & $\begin{array}{l}\text { Survival disappointing in } \\
\text { M1 disease started only } \\
\text { on LT ADT, despite active } \\
\text { treatments available at first } \\
\text { ADT failure. Spend most } \\
\text { of their time in CR relapse. }\end{array}$ \\
\hline $\begin{array}{l}\text { Singh, } 2004 \\
{[149]}\end{array}$ & 3iii/A & $\mathrm{R}$ & 30 & $\begin{array}{l}\leq 5 \text { met } \\
\text { lesions }\end{array}$ & $\begin{array}{l}\text { External } \\
\text { RT }\end{array}$ & $\begin{array}{l}\text { 5- and } 10 \mathrm{yr} \text { OS- } 73 \% \\
\text { and } 36 \% \text { in OM, as } \\
\text { compared to } 45 \% \text { and } \\
18 \% \text { in those with }>5 \\
\text { met lesions }\end{array}$ & $\begin{array}{l}\text { Findings suggest early } \\
\text { detection and aggressive } \\
\text { treatment is worth testing } \\
\text { to improve long-term } \\
\text { survival }\end{array}$ \\
\hline $\begin{array}{l}\text { Engel, } 2010 \\
{[101]}\end{array}$ & $3 \mathrm{iii} / \mathrm{A}$ & $\mathrm{R}$ & 938 & $+\mathrm{LNs}$ & $+/-\mathrm{RP}$ & \begin{tabular}{|l|}
$5 \mathrm{yr}-$ and $10 \mathrm{yr}$ OS- \\
$84 \%$ and $64 \%$ with \\
completed RP, and \\
$60 \%$ and $28 \%$, with \\
aborted RP. PCa- \\
specific survival at 5- \\
and 10 -yrs- $95 \%$ and \\
$86 \%$, with completed \\
RP and was $70 \%$ and \\
$40 \%$, with aborted RP
\end{tabular} & $\begin{array}{l}\text { Abandonment of RP in } \\
\text { men with positive LNs } \\
\text { may not be appropriate }\end{array}$ \\
\hline
\end{tabular}

(Continued) 


\begin{tabular}{|c|c|c|c|c|c|c|c|}
\hline $\begin{array}{l}\text { 1st Author, } \\
\text { Year [Ref] }\end{array}$ & $\begin{array}{l}\text { Strength } \\
\text { of } \\
\text { evidence- } \\
\text { based } \\
\text { on study } \\
\text { design / } \\
\text { endpoints }\end{array}$ & $\begin{array}{l}\text { Prospective } \\
\text { (P) / } \\
\text { Retrospective } \\
\text { (R) }\end{array}$ & $\begin{array}{l}\text { Sample } \\
\text { size }\end{array}$ & $\begin{array}{l}\text { Definition- } \\
\text { Oligometa } \\
\text { stases }\end{array}$ & Therapy & Endpoints & Conclusion \\
\hline $\begin{array}{l}\text { Tabata, } 2012 \\
{[148]}\end{array}$ & 3iii/A & $\mathrm{R}$ & 35 & $\begin{array}{l}<6 \text { bone mets } \\
\text { on bone scan, } \\
\text { each site less } \\
\text { than } 50 \% \\
\text { the size of } \\
\text { a vertebral } \\
\text { body }\end{array}$ & RT & $\begin{array}{l}3 \mathrm{yr} \text { OS- } 77 \% ; 14 / 16 \\
(87 \%) \text { of pats who } \\
\text { had pain were } \\
\text { improved } 1 \mathrm{mth} \text { after } \\
\text { RT; median duration } \\
\text { of pain control } 12 \\
\text { month }\end{array}$ & $\begin{array}{l}\text { RT for bone } \mathrm{OM} \text { in } \mathrm{PCa} \\
\text { was effective for long-term } \\
\text { pain relief }\end{array}$ \\
\hline $\begin{array}{l}\text { Schick, } 2013 \\
{[141]}\end{array}$ & 3iii /A & $\mathrm{R}$ & 50 & $\begin{array}{l}1-4 \text { mets, } \\
\text { synchronous } \\
\text { or } \\
\text { metachronous }\end{array}$ & $\begin{array}{l}\text { Mets- } \\
\text { ADT and } \\
\text { HDRT }\end{array}$ & $\begin{array}{l}\text { 3yr biochemical } \\
\text { relapse-free survival } \\
\text { (bRFS), clinical } \\
\text { failure-free survival, } \\
\text { and OS- } 54.5,58.6 \text {, } \\
\text { and } 92 \%\end{array}$ & $\begin{array}{l}\text { OM may be treated } \mathrm{w} \\
\text { short ADT and HDRT } \\
\text { to the met regions. High } \\
\text { dose improves bRFS. } \\
\text { May prolong failure- } \\
\text { free interval between } 2 \\
\text { consecutive ADT courses. }\end{array}$ \\
\hline $\begin{array}{l}\text { Ponti, } 2014 \\
{[143]}\end{array}$ & 3iii/A & $\mathrm{R}$ & 16 & $\begin{array}{l}\text { Distant } \\
\text { relapse in } \\
\text { a limited } \\
\text { number of } \\
\text { regions, } \leq 5 \\
\text { mets }\end{array}$ & $\begin{array}{l}\text { SBRT } \\
+/-\mathrm{HT}\end{array}$ & $\begin{array}{l}\text { Local control, } \\
\text { biochemical PFS, } \\
\text { OS, toxicity. OS at } \\
29 \text { mths } 95 \% \text { Distant } \\
\text { relapse in a limited } \\
\text { number of regions, } \\
\leq 5 \text { mets }\end{array}$ & $\begin{array}{l}\text { SBRT safe, effective, } \\
\text { minimally invasive in } \\
\text { limited LN recurrence in } \\
\text { OMPCa }\end{array}$ \\
\hline $\begin{array}{l}\text { Jereczek- } \\
\text { Fossa, } 2014 \\
{[147]}\end{array}$ & 3iii/A & $\mathrm{R}$ & 69 & $\begin{array}{l}\text { Single } \\
\text { abdominal LN } \\
\text { recurrence }\end{array}$ & SBRT & $\begin{array}{l}3-y r \text { in-field PFS, } \\
\text { PFS, OS- } 64 \%, \\
11.7 \% \text {, and } 50 \%\end{array}$ & $\begin{array}{l}\text { SBRT is feasible for single } \\
\text { abdominal LN recurrence, } \\
\text { offering excellent in-field } \\
\text { tumor control. }\end{array}$ \\
\hline $\begin{array}{l}\text { Cadeddu, } \\
1997 \text { [132] }\end{array}$ & 3 iii /B & $\mathrm{R}$ & 38 & $\begin{array}{l}+\mathrm{LN} \text { : pelvic } \\
\text { lymph } \\
\text { adenopathy }\end{array}$ & $\begin{array}{l}\text { PLND } \\
+/-\mathrm{RP}\end{array}$ & $\begin{array}{l}\text { PCa-specific survival } \\
\text { at } 5-\text { and } 10-y r s- \\
(93 \% \text { and } 56 \% \text { in the } \\
\text { PLND/RP group and } \\
58 \% \text { and } 34 \% \text { in the } \\
\text { PLND group }\end{array}$ & $\begin{array}{l}\mathrm{RP}, \text { as compared to } \\
\text { conservative therapy, may } \\
\text { prolong survival }\end{array}$ \\
\hline $\begin{array}{l}\text { Ahmed, } 2013 \\
{[145]}\end{array}$ & 3iii/B & $\mathrm{R}$ & 17 & $\begin{array}{l}\leq 5 \text { met } \\
\text { lesions }\end{array}$ & SBRT & $\begin{array}{l}\text { Local control- } 100 \% \\
\text { at } 6 \mathrm{mo} \text {; cancer } \\
\text { specific survival } \\
\text { (CSS)-6- and } 12 \mathrm{mo-} \\
100 \% \text {; freedom from } \\
\text { distant progression } \\
\text { (FFDP)- } 6-\text { and } \\
12 \mathrm{mo}-74 \%, 40 \%\end{array}$ & $\begin{array}{l}\text { Excellent LC with } \\
\text { SBRT for OM PCa; over } \\
50 \% \text { patients achieved } \\
\text { undetectable PSA after } \\
\text { SBRT }\end{array}$ \\
\hline $\begin{array}{l}\text { Ost, } 2014 \\
{[142]}\end{array}$ & 3 iii /B & $\mathrm{R}$ & 80 & $\begin{array}{l}\text { Metachronous } \\
\text { mets }\end{array}$ & $\begin{array}{l}\text { Mets- } \\
\text { ADT, } \\
\text { AS, or } \\
\text { MDT }\end{array}$ & $\begin{array}{l}\text { Median PCSS- } 6.6 \\
\text { yrs. }\end{array}$ & $\begin{array}{l}\text { Longer PSA DT, } \\
\text { involvement of nodes or } \\
\text { axial skeleton and lower } \\
\text { \# mets assoc w improved } \\
\text { PCSS. }\end{array}$ \\
\hline $\begin{array}{l}\text { Decaestecker, } \\
2014[144]\end{array}$ & 3iii/B & $\mathrm{R}$ & 50 & $\begin{array}{l}\leq 3 \\
\text { metachronous } \\
\text { asymptomatic } \\
\text { mets }\end{array}$ & $\begin{array}{l}\text { SBRT } \\
(2 \text { RT } \\
\text { schedules } \\
\text { used })+/- \\
\text { HT }\end{array}$ & $\begin{array}{l}\text { Median PFS- 19mo; } \\
\text { median ADT-FS- } 25 \\
\text { month; } 2-, 5 \mathrm{yr} \text { PCSS- } \\
96 \%, 90 \%\end{array}$ & $\begin{array}{l}\text { Repeated SBRT for OM } \\
\text { PCa postpones palliative } \\
\text { ADT }\end{array}$ \\
\hline
\end{tabular}

(Continued) 


\begin{tabular}{|c|c|c|c|c|c|c|c|}
\hline $\begin{array}{l}\text { 1st Author, } \\
\text { Year [Ref] }\end{array}$ & $\begin{array}{l}\text { Strength } \\
\text { of } \\
\text { evidence- } \\
\text { based } \\
\text { on study } \\
\text { design / } \\
\text { endpoints }\end{array}$ & $\begin{array}{l}\text { Prospective } \\
\text { (P) / } \\
\text { Retrospective } \\
\text { (R) }\end{array}$ & $\begin{array}{l}\text { Sample } \\
\text { size }\end{array}$ & $\begin{array}{l}\text { Definition- } \\
\text { Oligometa } \\
\text { stases }\end{array}$ & Therapy & Endpoints & Conclusion \\
\hline $\begin{array}{l}\text { Berkovic, } \\
2013 \text { [146] }\end{array}$ & 3iii/Di & $\mathrm{R}$ & 24 & $\begin{array}{l}\text { Biochemical } \\
\text { recurrence } \\
\text { after curative } \\
\text { treatment } \\
\text { to primary } \\
\text { (RP, RT, or } \\
\text { both), then } \leq 3 \\
\text { synchronous } \\
\text { asymptomatic } \\
\text { mets } \\
\end{array}$ & SBRT & $\begin{array}{l}\text { Androgen deprivation } \\
\text { therapy-free survival } \\
\text { (ADT-FS)- 1-, 2yr- } \\
82 \%, 54 \% \text {; clinical } \\
\text { progression free } \\
\text { survival- } 1-, 2 \mathrm{yr}-72 \% \\
\text { and } 42 \%\end{array}$ & $\begin{array}{l}\text { Repeated salvage SBRT } \\
\text { feasible, well tolerated, } \\
\text { and defers palliative ADT } \\
\text { with a median } 38 \mathrm{mo} \text { in } \\
\text { OMPca }\end{array}$ \\
\hline $\begin{array}{l}\text { Ost, } 2014 \\
{[133]}\end{array}$ & 3iii/Diii & $\mathrm{R}$ & 450 & $\begin{array}{l}\text { Metachronous } \\
\text { mets with } \\
\text { controlled } \\
\text { primary, }+ \\
\text { underwent } \\
\text { MDT for } \\
\text { recurrent PCa }\end{array}$ & $\begin{array}{l}\text { RT or } \\
\text { LND }\end{array}$ & $\begin{array}{l}\text { About } 50 \% \text { PFS at } \\
1-3 \text { yrs post-MDT }\end{array}$ & $\begin{array}{l}\text { MDT promising approach } \\
\text { for OM PCa recurrence but } \\
\text { low level of evidence }\end{array}$ \\
\hline
\end{tabular}

Abbreviations: M1=distant metastases; LT ADT=long-term androgen deprivation therapy; FFS=failure-free survival; $\mathrm{OS}=$ overall survival; $\mathrm{CR}=$ castrate resistance; met=metastasis; $\mathrm{RT}=$ radiation therapy; $\mathrm{OM}=\mathrm{oligometastasis}$; $\mathrm{LNs}=$ lymph nodes; $\mathrm{RP}=$ radical prostatectomy; $\mathrm{PCa}=$ prostate cancer; $\mathrm{HDRT}=$ high dose radiation therapy; $\mathrm{ADT}=$ androgen deprivation therapy; SBRT=stereotactic body radiation therapy; HT=hormone therapy; PFS=progression free survival; LN=lymph node; $\mathrm{OM} \mathrm{PCa}=$ oligometastatic prostate cancer; $\mathrm{PLND}=$ positive lymph node dissection; $\mathrm{LC}=$ local control; $\mathrm{PSA}=$ prostate specific antigen; PCSS=prostate cancer specific survival; $\mathrm{AS}=$ active surveillance; $\mathrm{MDT}=$ metastasis directed therapy; $\mathrm{DT}=\mathrm{doubling}$ time; ADT-FS=androgen deprivation free survival; $\mathrm{LND}=$ lymph node dissection; $\mathrm{PFS}=$ progression free survival

(Level A) were reported in seven studies: 10-year $(36 \%$ [149] and 64\% [101]), 3-year (50\% [147], 77\% [148], and $92 \%$ [141]), 29-month (95\%) [143], and 2-year $(72 \%)$ [137].

Currently there are two studies, not yet completed, for which we are anxiously awaiting the results. Decaestecker et al. are conducting the first randomized, phase 2 trial on men with an oligometastatic recurrence to assess the impact of metastases-directed therapy - versus active surveillance - on the start of palliative ADT [150]. Attard et al., as part of the ongoing STAMPEDE trial, describes the addition of a new treatment arm (enzalutamide, abiraterone and prednisone with ADT) for men with newly diagnosed M1 disease [151].

\section{Current trials of oligometastatic disease}

We reviewed clinicaltrials.gov to gauge the ongoing studies for oligometastatic disease. Multiple trials are underway to determine if the treatment of oligometastatic disease is beneficial in cancer as reported on clinicaltrials. gov [152] (see Table 12).

Nine studies are in progress for oligometastatic NSCLC, with therapies ranging from
SBRT [153-155], to RT [156, 157], to chemoradiation therapy [158], to targeted therapy +/- RT [159], to immunotherapy [160], to any local therapy [161]. For men with oligometastatic prostate cancer, nine trials are underway investigating a variety of therapies including single agent RT [162-165], RT in combination with HT [166-168], salvage treatment (surgical or RT) of metastases [169], and combination of HT + immunotherapy [170]. For women with oligometastatic breast cancer, six studies are currently open investigating several therapies including various chemotherapy regimens [171], SABR + antibodies [172], SBRT [173], chemotherapy + antibodies + RT [174], targeted therapies [175] and RT [176]. Three studies are studying oligometastatic melanoma, two are investigating immunotherapy + SABT/SRS $[177,178]$ and the third study is investigating chemotherapy with and without surgery [179]. There are thirteen studies enrolling patients with oligometastases without a specific primary tumor [180-192], employing a variety of treatment options. Finally, there is one study each for oligometastatic colorectal cancer using chemoradiation [193] and for oligometastatic pediatric sarcoma using SBRT [194]. 
Table 12: Current studies for oligometastatic disease (ClinicalTrials.gov (CTG) as of 2/1/2015)

\begin{tabular}{|c|c|c|c|c|c|}
\hline CTG NCT\# / site & Condition & Name & Purpose & Algorithm & $\begin{array}{l}\text { Primary Outcome/ } \\
\text { Endpoint }\end{array}$ \\
\hline $\begin{array}{l}01859221 \\
\text { University Florida } \\
{[162]}\end{array}$ & $\begin{array}{l}\text { Prostate } \\
\text { oligometastases }\end{array}$ & $\begin{array}{l}\text { Radiotherapy for } \\
\text { OMPC }\end{array}$ & $\begin{array}{l}\text { Phase II study to } \\
\text { evaluate outcomes of } \\
\text { patients treated with } \\
\text { Stereotactic radiation } \\
\text { therapy for OMPC }\end{array}$ & RT & $\begin{array}{l}\text { Improved PFS over } \\
\text { historic controls. }\end{array}$ \\
\hline $\begin{array}{l}01777802 \\
\text { Mayo Clinic [163] }\end{array}$ & $\begin{array}{l}\text { Prostate } \\
\text { oligometastases }\end{array}$ & $\begin{array}{l}\text { Monitoring Anti- } \\
\text { Prostate Cancer } \\
\text { Immunity Following } \\
\text { SBRT }\end{array}$ & $\begin{array}{l}\text { Determine if } \\
\text { SBRT conditions } \\
\text { solid tumors to be } \\
\text { favorable to the } \\
\text { initiation of robust } \\
\text { antitumoral immune } \\
\text { responses }\end{array}$ & $\begin{array}{l}\text { Observation } \\
\text { following SBRT }\end{array}$ & $\begin{array}{l}\text { Induction of anti- } \\
\text { prostate cancer } \\
\text { immunity }\end{array}$ \\
\hline $\begin{array}{l}02020070 \\
\text { MSKCC [170] }\end{array}$ & $\begin{array}{l}\text { Prostate } \\
\text { oligometastases }\end{array}$ & $\begin{array}{l} \\
\text { Ipilimumab, } \\
\text { degarelix, + RP in } \\
\text { castrate sensitive } \\
\text { PC or ipilimumab } \\
+ \text { degarelix in } \\
\text { biochemical } \\
\text { recurrent castrate } \\
\text { sensitive PC after RP }\end{array}$ & $\begin{array}{l}\text { Assess safety } \\
+ \text { efficacy of } \\
\text { combining HT }+ \\
\text { immunotherapy } \\
\text { in non-castrate } \\
\text { resistant PC. Cohort } \\
\text { 1: ipilimumab + } \\
\text { degarelix pre- and } \\
\text { post- RP in newly } \\
\text { diagnose OM } \\
\text { castrate-sensitive } \\
\text { disease. Cohort 2: } \\
\text { post definitive local } \\
\text { therapy with RP, but } \\
\text { with biochemical } \\
\text { recurrence }\end{array}$ & $\begin{array}{l}\text { IT, LHRH antagonist } \\
+ \text { surgery } \\
\text {-OR- } \\
\text { IT, LHRH antagonist }\end{array}$ & $\begin{array}{l}\text { Undetectable PSA } \\
\text { at 12- and } 20 \text {-mths } \\
\text { with non-castrate } \\
\text { testosterone. }\end{array}$ \\
\hline $\begin{array}{l}02264379 \\
\text { Technische U } \\
\text { Dresden [165] }\end{array}$ & $\begin{array}{l}\text { Prostate } \\
\text { oligometastases }\end{array}$ & $\begin{array}{l}\text { Percutaneous high- } \\
\text { dose RT in OMPC }\end{array}$ & $\begin{array}{l}\text { Evaluate outcomes } \\
\text { of patients treated } \\
\text { with high-dose } \\
\text { radiation using either } \\
\text { hypofractionated } \\
\text { or normofractionated } \\
\text { RT; to establish } \\
\text { efficacy + safety }\end{array}$ & $\begin{array}{l}\text { HDRT Hypofraction } \\
\text {-OR- } \\
\text { Normofraction }\end{array}$ & Toxicity \\
\hline $\begin{array}{l}01558427 \\
\text { UHosp, Ghent [169] }\end{array}$ & $\begin{array}{l}\text { Prostate } \\
\text { oligometastases }\end{array}$ & $\begin{array}{l}\text { Salvage treatment } \\
\text { of active clinical } \\
\text { surveillance for } \\
\text { OMPC: PhII RCT }\end{array}$ & $\begin{array}{l}\text { To determine if } \\
\text { salvage treatment of } \\
\text { OMPC with either } \\
\text { surgery or RT might } \\
\text { postpone the start of } \\
\text { ADT }\end{array}$ & $\begin{array}{l}\text { Surgery } \\
\text {-OR- } \\
\text { RT, Which delays } \\
\text { ADT? }\end{array}$ & ADT-free survival \\
\hline $\begin{array}{l}02192788 \\
\text { Hospital Provincial } \\
\text { de Castellon [164] }\end{array}$ & $\begin{array}{l}\text { Prostate } \\
\text { oligometastases }\end{array}$ & $\begin{array}{l}\text { PhII study SBRT as } \\
\text { treatment for OMPC }\end{array}$ & $\begin{array}{l}\text { Evaluate effect } \\
\text { SBRT for OMPC, } \\
\text { regardless of basal } \\
\text { treatment received }\end{array}$ & SBRT & $\begin{array}{l}\text { \# patients without } \\
\text { progression of PC } \\
\text { treated by SBRT }\end{array}$ \\
\hline $\begin{array}{l}02274779 \\
\text { Institut cancerologie } \\
\text { de l'Ouest [166] }\end{array}$ & $\begin{array}{l}\text { Prostate } \\
\text { oligometastases }\end{array}$ & $\begin{array}{l}\text { PHII trial salvage RT } \\
+ \text { HT in OM pelvic } \\
\text { node relapses of PC }\end{array}$ & $\begin{array}{l}\text { Assess BC or } \\
\text { clinical relapse-free } \\
\text { survival at } 2 \text { yrs } \\
\text { of PC with } 1-5 \\
\text { OM treated with } \\
\text { concomitant HDCRT } \\
+ \text { HT } \\
\end{array}$ & $\mathrm{RT}+\mathrm{HT}$ & $\begin{array}{l}\mathrm{BC} \text { or clinical } \\
\text { relapse-free survival } \\
\text { at } 2 \mathrm{yrs}\end{array}$ \\
\hline $\begin{array}{l}00544830 \\
\text { Institute CoHMCNC } \\
{[167]}\end{array}$ & $\begin{array}{l}\text { Prostate } \\
\text { oligometastases }\end{array}$ & $\begin{array}{l}\text { IMRT in treating } \\
\text { patients undergoing } \\
\text { ADT for } \mathrm{mPC}\end{array}$ & $\begin{array}{l}\text { Assess how well } \\
\text { IMRT works in pats } \\
\text { undergoing ADT } \\
\text { for mPC }\end{array}$ & $\mathrm{HT}+\mathrm{RT}$ & Time to PSA relapse \\
\hline
\end{tabular}

(Continued) 


\begin{tabular}{|c|c|c|c|c|c|}
\hline CTG NCT\# / site & Condition & Name & Purpose & Algorithm & $\begin{array}{l}\text { Primary Outcome/ } \\
\text { Endpoint }\end{array}$ \\
\hline $\begin{array}{l}00544830 \\
\text { City of Hope } \\
\text { Medical Center } \\
{[168]}\end{array}$ & $\begin{array}{l}\text { Prostate } \\
\text { oligometastases }\end{array}$ & $\begin{array}{l}\text { IMRT in Patients } \\
\text { Undergoing ADT for } \\
\text { Metastatic Prostate } \\
\text { Cancer }\end{array}$ & $\begin{array}{l}\text { Evaluate intensity- } \\
\text { modulated radiation } \\
\text { therapy works in } \\
\text { treating patients } \\
\text { undergoing androgen } \\
\text { deprivation therapy } \\
\text { for metastatic } \\
\text { prostate cancer }\end{array}$ & ADT + IMRT & Time to PSA relapse \\
\hline $\begin{array}{l}01728779 \\
\text { Sidney Kimmel } \\
\text { Comprehensive } \\
\text { Cancer center }[180]\end{array}$ & $\begin{array}{l}\text { Oligometastases to } \\
\text { lung, liver, bone }\end{array}$ & $\begin{array}{l}\text { Stereotactic Body } \\
\text { Radiation With } \\
\text { Nelfinavir for } \\
\text { Oligometastases }\end{array}$ & $\begin{array}{l}\text { Evaluate efficacy } \\
\text { radiosensitizer } \\
\text { nelfinavir used } \\
\text { concurrently with } \\
\text { SBRT }\end{array}$ & Nelfinavir + SBRT & PFS at 6 months \\
\hline $\begin{array}{l}01345539 \\
\text { U Pittsburgh [181] }\end{array}$ & $\begin{array}{l}\text { Oligometastatic } \\
\text { disease }\end{array}$ & $\begin{array}{l}\text { Radiosurgery for } \\
\text { OM Disease at } \\
\text { Initial Presentation }\end{array}$ & $\begin{array}{l}\text { Evaluate feasibility } \\
\text { of radiosurgery for } \\
\text { all metastatic sites } \\
\text { in OM }\end{array}$ & SRS & $\begin{array}{l}\text { Feasibility SRS/ } \\
\text { SBRT in OM at } \\
\text { initial presentation }\end{array}$ \\
\hline $\begin{array}{l}02076477 \\
\text { Sichuan Cancer } \\
\text { Hospital + Research } \\
\text { Institute [158] }\end{array}$ & $\begin{array}{l}\text { OM stage IV } \\
\text { NSCLC }\end{array}$ & $\begin{array}{l}\text { The Optimal } \\
\text { Intervention Time } \\
\text { of Radiotherapy } \\
\text { for OM Stage IV } \\
\text { NSCLC }\end{array}$ & $\begin{array}{l}\text { Evaluates optimal } \\
\text { time for RT for OM } \\
\text { stage IV lung cancer }\end{array}$ & RT & $\begin{array}{l}\text { Short-term effects } \\
\text { (response rate using } \\
\text { RECIST) }\end{array}$ \\
\hline $\begin{array}{l}01796288 \\
\text { Wu Jieping Medical } \\
\text { Foundation [159] }\end{array}$ & OM NSCLC & $\begin{array}{l}\text { Radiotherapy in } \\
\text { Oligometastatic } \\
\text { Non-squamous } \\
\text { NSCLC With } \\
\text { Clinical Benefits } \\
\text { From 2nd Line } \\
\text { Erlotinib } \\
\end{array}$ & $\begin{array}{l}\text { Evaluate RT in } \\
\text { combination with } \\
\text { erlotinib in OM } \\
\text { NSCLC }\end{array}$ & Erlotinib +/- RT & PFS \\
\hline $\begin{array}{l}01345552 \\
\text { U Pittsburgh [182] }\end{array}$ & $\begin{array}{l}\text { Recurrent OM } \\
\text { disease }\end{array}$ & $\begin{array}{l}\text { Radiosurgery for } \\
\text { Patients Recurrent } \\
\text { OM Disease } \\
\end{array}$ & $\begin{array}{l}\text { Evaluate feasibility } \\
\text { of SRS in recurrent } \\
\text { OM disease }\end{array}$ & SRS & $\begin{array}{l}\text { Being able to } \\
\text { complete accrual to } \\
\text { study }\end{array}$ \\
\hline $\begin{array}{l}01565837 \\
\text { Comprehensive } \\
\text { Cancer Centers } \\
\text { Nebraska [177] }\end{array}$ & OM Melanoma & \begin{tabular}{|l|} 
Concurrent \\
Ipilimumab and \\
Stereotactic Ablative \\
Radiation Therapy \\
(SART) for OM \\
Unresectable \\
Melanoma \\
\end{tabular} & $\begin{array}{l}\text { Evaluate if } \\
\text { SART + ipilimumab } \\
\text { will improve } \\
\text { survival in OM } \\
\text { melanoma }\end{array}$ & Ipilimumab + SART & $\begin{array}{l}\text { Safety and } \\
\text { tolerability }\end{array}$ \\
\hline $\begin{array}{l}02316002 \\
\text { U Pennsylvania } \\
{[160]}\end{array}$ & OM NSCLC & \begin{tabular}{|l|} 
Phase II Study of \\
Pembrolizumab \\
After Curative Intent \\
Treatment for OM \\
NSCLC \\
\end{tabular} & $\begin{array}{l}\text { Evaluate how well } \\
\text { pembrolizumab } \\
\text { works in previously } \\
\text { treated OM NSCLC }\end{array}$ & Pembrolizumab & PFS \\
\hline $\begin{array}{l}01646034 \\
\text { Netherlands Cancer } \\
\text { Institute [171] }\end{array}$ & OM Breast cancer & $\begin{array}{l}\text { High Dose CT in } \\
\text { OM Homologous } \\
\text { Recombination } \\
\text { Deficient Breast } \\
\text { Cancer }\end{array}$ & $\begin{array}{l}\text { Studies effect of } \\
\text { high-dose alkylating } \\
\text { CT versus standard } \\
\text { CT in OM breast } \\
\text { cancer with } \\
\text { recombination } \\
\text { deficiency }\end{array}$ & $\begin{array}{l}\text { Carboplatin, } \\
\text { thiotepa, and } \\
\text { cyclophosphamide } \\
\text { versus docetaxel, } \\
\text { doxorubicin, } \\
\text { cyclophosphamide, } \\
\text { carboplatin, } \\
\text { paclitaxel, } \\
\text { gemcitabine } \\
\end{array}$ & Event free survival \\
\hline $\begin{array}{l}02228356 \\
\text { Universitair } \\
\text { Zeikenhuis Brussel } \\
{[183]}\end{array}$ & Neoplasm metastasis & $\begin{array}{l}\text { Non-interventional } \\
\text { Observational Study } \\
\text { of SBRT for OM } \\
\text { Cancer }\end{array}$ & $\begin{array}{l}\text { Investigate if } \\
\text { respiration control } \\
\text { and improved } \\
\text { technique improve } \\
\text { local control }\end{array}$ & SBRT & 1-year local control \\
\hline
\end{tabular}

(Continued) 


\begin{tabular}{|c|c|c|c|c|c|}
\hline CTG NCT\# / site & Condition & Name & Purpose & Algorithm & $\begin{array}{l}\text { Primary Outcome/ } \\
\text { Endpoint }\end{array}$ \\
\hline $\begin{array}{l}01725165 \\
\text { M.D. Anderson } \\
\text { Cancer Center [161] }\end{array}$ & OM Lung cancer & OM Disease & $\begin{array}{l}\text { Learn if surgery or } \\
\text { radiation after CT } \\
\text { help control NSCLC }\end{array}$ & $\begin{array}{l}+/- \text { local } \\
\text { consolidation } \\
\text { therapy }\end{array}$ & PFS \\
\hline $\begin{array}{l}02303366 \\
\text { Peter MacCallum } \\
\text { Cancer Center, } \\
\text { Australia [172] }\end{array}$ & OM breast cancer & $\begin{array}{l}\text { Pilot Study SABR } \\
\text { for OM Breast } \\
\text { Neoplasia in } \\
\text { Combination With } \\
\text { the Anti-PD-1 } \\
\text { Antibody MK-3475 } \\
\end{array}$ & $\begin{array}{l}\text { Assess safety }+ \\
\text { feasibility of MK- } \\
3475+\text { SABR for } \\
\text { definitive treatment } \\
\text { of OM breast cancer }\end{array}$ & SABR + MK-3475 & $\begin{array}{l}\text { \# of patients who } \\
\text { complete treatment; } \\
\text { safety }\end{array}$ \\
\hline $\begin{array}{l}01759238 \\
\text { Universitatsklinikum } \\
\text { Hamburg-Eppendorf } \\
{[193]}\end{array}$ & OM CRC metastases & $\begin{array}{l}\text { Chemoradiotherapy } \\
\text { for Patients With } \\
\text { Oligometastatic } \\
\text { Colorectal Cancer }\end{array}$ & $\begin{array}{l}\text { Evaluate role of CRT } \\
\text { with capecitabine } \\
\text { and bevacizumab in } \\
\text { OM patients who are } \\
\text { neither progressive } \\
\text { nor resectable after } \\
\text { CT } \\
\end{array}$ & $\begin{array}{l}\text { Capecitabine, } \\
\text { bevacizumab and RT }\end{array}$ & PFS \\
\hline $\begin{array}{l}01282450 \\
\text { Maastricht Radiation } \\
\text { Oncology [157] }\end{array}$ & $\begin{array}{l}\text { Stage IV OM } \\
\text { NSCLC }\end{array}$ & \begin{tabular}{|l|} 
Concurrent and \\
Non-concurrent CRT \\
or RT Alone for OM \\
Stage IV NSCLC \\
\end{tabular} & $\begin{array}{l}\text { Aim is to improve } \\
\text { long term survival in } \\
\text { OM NSCLC }\end{array}$ & RT & $\begin{array}{l}\text { OS } \\
\text { Note: Study } \\
\text { completed }\end{array}$ \\
\hline $\begin{array}{l}02086721 \\
\text { Maastricht Radiation } \\
\text { Oncology [188] }\end{array}$ & Solid tumors & $\begin{array}{l}\text { Assess Toxicity of } \\
\text { Immunocytokine } \\
\text { L19-IL2 After } \\
\text { SABR OM Solid } \\
\text { Tumour }\end{array}$ & $\begin{array}{l}\text { Hypothesis is that } \\
\text { the immunocytokine } \\
\text { L19-IL2 and RT will } \\
\text { synergize to improve } \\
\text { OS in OM solid } \\
\text { tumors }\end{array}$ & L19-IL2 + RT & Toxicity \\
\hline $\begin{array}{l}01965223 \\
\text { Trans-Tasman } \\
\text { Radiation Oncolog } \\
\text { Group [189] }\end{array}$ & $\begin{array}{l}\text { Cancer metastases } \\
\text { to lung }\end{array}$ & $\begin{array}{l}\text { Randomized Phase } \\
\text { II Study SABR } \\
\text { Metastases to the } \\
\text { Lung }\end{array}$ & $\begin{array}{l}\text { Determine safety of } \\
\text { SABR versus SRS } \\
\text { for OM to lung }\end{array}$ & $\begin{array}{l}\text { Multi-fraction SABR } \\
\text { and single fraction } \\
\text { SABR }\end{array}$ & Toxicity \\
\hline $\begin{array}{l}02107755 \\
\text { Ohio State U } \\
\text { Comprehensive } \\
\text { Cancer Center [178] }\end{array}$ & $\begin{array}{l}\text { Metastatic } \\
\text { melanoma }\end{array}$ & $\begin{array}{l}\text { Stereotactic RT } \\
\text { and Ipilimumab } \\
\text { in Metastatic } \\
\text { Melanoma }\end{array}$ & $\begin{array}{l}\text { Determine if } \\
\text { stereotactic } \\
\text { radiosurgery }+ \\
\text { ipilimumab kills } \\
\text { more tumor cells by } \\
\text { causing additional } \\
\text { melanoma antigens } \\
\text { to be presented to } \\
\text { immune system, }\end{array}$ & $\begin{array}{l}\text { Ipilimumab } \\
+ \text { stereotactic } \\
\text { radiosurgery }\end{array}$ & PFS \\
\hline $\begin{array}{l}01446744 \\
\text { Lawson Health } \\
\text { Research institute } \\
{[190]}\end{array}$ & Metastatic tumors & \begin{tabular}{|l} 
SABR for \\
Comprehensive \\
Treatment of OM \\
Tumors \\
\end{tabular} & $\begin{array}{l}\text { Compare SABR with } \\
\text { CT and conventional } \\
\text { RT to assess impact } \\
\text { on OS and Q of L }\end{array}$ & $\begin{array}{l}\text { SABR versus } \\
\text { palliative RT }\end{array}$ & OS \\
\hline $\begin{array}{l}02264886 \\
\text { Washington } U \\
\text { School of Medicine } \\
{[191]}\end{array}$ & $\begin{array}{l}\text { Central thorax } \\
\text { cancer, liver } \\
\text { cancer, or non-liver } \\
\text { abdominal cancer }\end{array}$ & $\begin{array}{l}\text { Adaptive MRI- } \\
\text { Guided SBRT } \\
\text { for Unresectable } \\
\text { Primary or OM } \\
\text { Central Thorax } \\
\text { and Abdominal } \\
\text { Malignancies } \\
\end{array}$ & $\begin{array}{l}\text { Assess feasibility } \\
\text { of RT using MRI- } \\
\text { guided adaptive } \\
\text { technique (day by } \\
\text { day re-planning } \\
\text { while patient is } \\
\text { receiving treatment) }\end{array}$ & MRI guided SBRT & $\begin{array}{l}\text { Feasibility of } \\
\text { MRI-guided SBRT } \\
\text { (treatment can be } \\
\text { delivered in }<80 \\
\text { minutes for }>75 \% \text { of } \\
\text { patients) }\end{array}$ \\
\hline $\begin{array}{l}01761929 \\
\text { Princess Margaret } \\
\text { Hospital, Canada } \\
{[184]}\end{array}$ & OM solid tumors & $\begin{array}{l}5 \text { Fraction SBRT for } \\
\text { OM Regimen, for } \\
\text { Extra-Cranial OM }\end{array}$ & $\begin{array}{l}\text { Monitor side effects } \\
\text { and outcomes from } \\
\text { higher doses of RT, } \\
\text { while limiting dose } \\
\text { to normal tissues } \\
\text { (using a } 5 \text { day } \\
\text { schedule) }\end{array}$ & RT in 5 fractions & $\begin{array}{l}1 \text { year PFS at index } \\
\text { site }\end{array}$ \\
\hline
\end{tabular}

(Continued) 


\begin{tabular}{|c|c|c|c|c|c|}
\hline CTG NCT\# / site & Condition & Name & Purpose & Algorithm & $\begin{array}{l}\text { Primary Outcome/ } \\
\text { Endpoint }\end{array}$ \\
\hline $\begin{array}{l}02170181 \\
\text { U of Texas SW } \\
\text { Medical Center } \\
{[185]}\end{array}$ & $\begin{array}{l}\text { Cancer and receiving } \\
\text { RT }\end{array}$ & $\begin{array}{l}\text { Prospective } \\
\text { Clinical Registry } \\
\text { for OM Disease, } \\
\text { Consolidation } \\
\text { Therapy, Debulking } \\
\text { Prior to CT, or Re- } \\
\text { Irradiation } \\
\end{array}$ & $\begin{array}{l}\text { Determine trends } \\
\text { in patterns of care } \\
\text { and outcomes for } \\
\text { refinement and } \\
\text { justification of this } \\
\text { treatment }\end{array}$ & SBRT & Patterns of care \\
\hline $\begin{array}{l}02089100 \\
\text { Gustave Roussy, } \\
\text { Cancer Campus, } \\
\text { Grand Paris [173] }\end{array}$ & Breast cancer & $\begin{array}{l}\text { Trial of Superiority } \\
\text { of SBRT in Breast } \\
\text { Cancer }\end{array}$ & $\begin{array}{l}\text { Prospectively study } \\
\text { role of metastases } \\
\text { SBRT with curative } \\
\text { intent in de novo } \\
\text { OM disease }\end{array}$ & $\begin{array}{l}\text { SBRT versus } \\
\text { placebo }\end{array}$ & PFS \\
\hline $\begin{array}{l}01898962 \\
\text { Rocky Mountain } \\
\text { Cancer Centers } \\
{[186]}\end{array}$ & $\begin{array}{l}\text { Stage IV or recurrent } \\
\text { carcinoma or } \\
\text { sarcoma }\end{array}$ & $\begin{array}{l}\text { Definitive Therapy } \\
\text { for OM Solid } \\
\text { Malignancies }\end{array}$ & $\begin{array}{l}\text { Aggressive treatment } \\
\text { to clinically active } \\
\text { sites of disease } \\
\text { (alone or }+ \text { systemic } \\
\text { therapy) may } \\
\text { improve survival }\end{array}$ & $\begin{array}{l}\text { Definitive } \\
\text { local treatment } \\
\text { (surgery, RT, } \\
\text { radioembolization) }\end{array}$ & $\begin{array}{l}\text { OS and disease- } \\
\text { specific survival }\end{array}$ \\
\hline $\begin{array}{l}00463060 \\
\text { Mount Sinai School } \\
\text { of Medicine [187] }\end{array}$ & Metastatic cancer & $\begin{array}{l}\text { Sutent and Radiation } \\
\text { as Treatment for } \\
\text { Limited Extent } \\
\text { Metastatic Cancer }\end{array}$ & $\begin{array}{l}\text { Combine sutent } \\
\text { and RT to } \\
\text { determine safety } \\
\text { and best method } \\
\text { of combining the } \\
\text { treatments }\end{array}$ & Sutent + RT & Survival \\
\hline $\begin{array}{l}02231775 \\
\text { M.D. Anderson } \\
\text { Cancer Center [179] }\end{array}$ & Stage IV melanoma & $\begin{array}{l}\text { Combi-Neo Study } \\
\text { for Stage IV } \\
\text { Melanoma }\end{array}$ & $\begin{array}{l}\text { Compare dabrafenib } \\
+ \text { trametinib before } \\
\text { surgery versus } \\
\text { surgery alone }\end{array}$ & $\begin{array}{l}\text { Surgery }+/- \\
\text { dabrafenib }+/- \text {, } \\
\text { trametinib }\end{array}$ & $\begin{array}{l}\text { 1-year relapse-free } \\
\text { survival }\end{array}$ \\
\hline $\begin{array}{l}01781741 \\
\text { Roswell Park cancer } \\
\text { Institute [154] }\end{array}$ & Stage III-IV NSCLC & $\begin{array}{l}\text { SBRT After Surgery } \\
\text { in Stage III-IV } \\
\text { Non-small Cell Lung } \\
\text { Cancer }\end{array}$ & $\begin{array}{l}\text { Evaluate SBRT post } \\
\text { lymphadenectomy }\end{array}$ & $\begin{array}{l}\text { Therapeutic } \\
\text { lymphadenectomy, } \\
\text { SBRT }\end{array}$ & Toxicity \\
\hline $\begin{array}{l}00776100 \\
\text { North Central } \\
\text { Cancer Treatment } \\
\text { Group/NCI [156] }\end{array}$ & Stage IV NSCLC & $\begin{array}{l}\text { RT or Observation } \\
\text { After CT in Stage IV } \\
\text { NSCLC }\end{array}$ & $\begin{array}{l}\text { Randomized Phase } \\
\text { II trial studying } \\
\text { how well RT works } \\
\text { compared to CT in } \\
\text { Stage IV NSCLC } \\
\end{array}$ & $\begin{array}{l}\text { RT versus } \\
\text { observation }\end{array}$ & $\begin{array}{l}\text { OS } \\
\text { Note: Study } \\
\text { completed }\end{array}$ \\
\hline $\begin{array}{l}00182793 \\
\text { City of Hope } \\
\text { Medical Center/NCI } \\
{[174]}\end{array}$ & Breast cancer & $\begin{array}{l}\text { Combination CT } \\
+/- \text { Trastuzumab } \\
\text { Followed By an } \\
\text { Autologous Stem } \\
\text { Cell Transplant } \\
\text { (SCT) and RT in } \\
\text { Stage III or IV } \\
\text { Breast Cancer } \\
\end{array}$ & \begin{tabular}{|l} 
Evaluate \\
combination CT \\
$+/-$ trastuzumab, \\
then autologous SCT \\
and RT
\end{tabular} & $\begin{array}{l}\mathrm{CT}+/- \text { Trastuzumab, } \\
\text { then SCT + RT }\end{array}$ & $\begin{array}{l}\text { TTP, OS, 5-year } \\
\text { response rate, } \\
\text { feasibility } \\
\text { Note: study } \\
\text { completed }\end{array}$ \\
\hline $\begin{array}{l}01941654 \\
\text { Chinese University } \\
\text { of Hong Kong [153] }\end{array}$ & $\begin{array}{l}\text { NSCLC activating } \\
\text { EGFR mutation }\end{array}$ & $\begin{array}{l}\text { ATOM_local } \\
\text { Ablative Therapy }\end{array}$ & \begin{tabular}{|l|} 
Efficacy of local \\
ablative therapy \\
in NSCLC with \\
activating EGFR \\
mutation with active \\
OM disease after 1st \\
line TKI EGFR \\
\end{tabular} & Local ablative RT & PFS at 1-year \\
\hline $\begin{array}{l}01185639 \\
\text { Comprehensive } \\
\text { Cancer Center Wake } \\
\text { Forest U [155] }\end{array}$ & NSCLC & $\begin{array}{l}\text { SBRT in Metastatic } \\
\text { NSCLC }\end{array}$ & $\begin{array}{l}\text { Evaluate feasibility, } \\
\text { safety, and efficacy } \\
\text { of SBRT after } \\
\text { 4cycles } 1 \text { st line CT }\end{array}$ & SBRT & PFS \\
\hline
\end{tabular}

(Continued) 


\begin{tabular}{|c|c|c|c|c|c|}
\hline CTG NCT\# / site & Condition & Name & Purpose & Algorithm & $\begin{array}{l}\text { Primary Outcome/ } \\
\text { Endpoint }\end{array}$ \\
\hline $\begin{array}{l}01763970 \\
\text { Sidney Kimmel } \\
\text { Comprehensive } \\
\text { Cancer Center [194] }\end{array}$ & Pediatric sarcoma & $\begin{array}{l}\text { SBRT for Pediatric } \\
\text { Sarcomas }\end{array}$ & $\begin{array}{l}\text { Evaluate efficacy of } \\
\text { SBRT ( } 5 \text { fractions) } \\
\text { in pediatric sarcoma }\end{array}$ & SBRT & SBRT efficacy \\
\hline $\begin{array}{l}01875666 \\
\text { UNC Lineberger } \\
\text { Comprehensive } \\
\text { Cancer Center [175] }\end{array}$ & Breast neoplasm & $\begin{array}{l}\text { Defining the } \\
\text { HER2 Positive } \\
(+) \text { Breast Cancer } \\
\text { Kinome Response } \\
\text { to Trastuzumab } \\
(\mathrm{T}), \text { Pertuzumab } \\
(\mathrm{P}), \text { Combination } \\
\text { Trastuzumab } \\
+ \text { Pertuzumab }(\mathrm{T}+\mathrm{P}) \text {, } \\
\text { or Combination } \\
\text { Trastuzumab }+ \\
\text { Lapatinib }(\mathrm{T}+\mathrm{L}) \\
\end{array}$ & $\begin{array}{l}\text { Evaluate kinome } \\
\text { response in Stage } \\
\text { I-IV HER2+ } \\
\text { scheduled to undergo } \\
\text { definitive therapy for } \\
\text { OM disease }\end{array}$ & $\begin{array}{l}\text { Randomized to } \mathrm{T}, \mathrm{P} \text {, } \\
\mathrm{T}+\mathrm{P} \text {, or } \mathrm{T}+\mathrm{L}\end{array}$ & $\begin{array}{l}\text { Difference in kinome } \\
\text { activation pre- and } \\
\text { post-treatment }\end{array}$ \\
\hline $\begin{array}{l}01706432 \\
\text { U of Chicago / NCI } \\
{[176]}\end{array}$ & $\begin{array}{l}\text { Stage IV breast } \\
\text { cancer }\end{array}$ & $\begin{array}{l}\text { Hypofractionated } \\
\text { IGRT in Stage IV } \\
\text { Breast Cancer }\end{array}$ & $\begin{array}{l}\text { Studies } \\
\text { hypofractionated } \\
\text { IGRT in stage IV } \\
\text { breast cancer }\end{array}$ & Hypofractionated RT & $\begin{array}{l}\text { Feasibility of } \\
\text { correlating number } \\
\text { of circulating tumor } \\
\text { cells with TTP in } \\
\text { metastatic breast } \\
\text { cancer } \\
\end{array}$ \\
\hline $\begin{array}{l}01347333 \\
\text { St. John's Mercy } \\
\text { Research Institute, } \\
\text { St. Louis [192] }\end{array}$ & $\begin{array}{l}\text { Liver metastases, } \\
\text { hepatocellular } \\
\text { carcinoma, } \\
\text { intrahepatic } \\
\text { cholangiocarcinoma }\end{array}$ & $\begin{array}{l}\text { SBRT for Liver } \\
\text { Tumors }\end{array}$ & $\begin{array}{l}\text { Evaluate local } \\
\text { control rate of SBRT } \\
\text { to liver tumors }\end{array}$ & SBRT & $\begin{array}{l}\text { Local tumor } \\
\text { recurrence rate }\end{array}$ \\
\hline
\end{tabular}

Abbreviations: $\mathrm{OMPC}=$ oligometastatic prostate cancer; $\mathrm{RT}=$ radiation therapy; $\mathrm{PFS}=$ progression free survival; $\mathrm{SBRT}=$ stereotactic body radiation therapy; $\mathrm{RP}=$ retropublic prostatectomy; $\mathrm{PC}=$ prostate cancer; $\mathrm{HT}=$ hormone therapy; $\mathrm{OM}=$ oligometastatic; IT=immunotherapy; LHRH=luteinizing hormone-releasing hormone; PSA=prostate specific antigen; $\mathrm{HD}=$ high dose; $\mathrm{RCT}=$ randomized controlled trial; $\mathrm{ADT}=$ androgen deprivation therapy; $\mathrm{BC}=$ biochemical; $\mathrm{IMRT}=\mathrm{image}$ guided radiation therapy; $\mathrm{PFS}=$ progression free survival; $\mathrm{SRS}=$ stereotactic radiosurgery; $\mathrm{CRT}=$ chemoradiation therapy; RECIST $=$ response evaluation criteria in solid tumors; $\mathrm{NSCLC}=$ non-small cell lung cancer; $\mathrm{CT}=$ chemotherapy; $\mathrm{OS}=$ overall survival; $\mathrm{SABR}=$ stereotactic ablative radiation therapy; $\mathrm{MRI}=$ magnetic resonance imaging; $\mathrm{SCT}=$ stem cell transplant; $\mathrm{TTP}=$ time to progression; $\mathrm{ATOM}=$ ablative therapy oligometastases; $\mathrm{EGFR}=$ epidermal growth factor receptor; $\mathrm{Q}$ of L=Quality of Life

\section{CONCLUSIONS}

Hellman proposed that tumors early in the chain of progression may give rise to metastases limited in number and location due to an undeveloped metastatic competence of the cancer "seeds" as well as the restrictive nature of the host tissue at some metastatic sites. The rationales of discrete steps of metastases $[11,12]$ and the hallmarks of cancer $[16,17]$ have both provided a biologic framework to support the oligometastatic state. Molecular diagnostics have not yet arrived to the point to determine where an individual metastatic deposit is within the continuum of malignancy $[32-44,55]$, preclinical models are beginning to lay the groundwork of the oligometastatic state [19-31].

While Hellman posited that oligometastasis may be underrecognized due to limitations in imaging diagnostics, imaging has advanced to such a degree that we are now in a yin-yang position such that, 1) imaging often likely demonstrates very early findings not likely to manifest into disease and, 2) imaging demonstrates very early disease that is destined to become widespread, which cannot be distinguished from oligometastases.

Hellman described potential cure as an attractive upshot of treating an oligometastatic state, but prolonged survival has been added as a potential benefit. There is an increasing shift toward individualized, multidisciplinary management of OM disease [34] because it is difficult to conduct randomized controlled trials in oligometastatic patients due to the variety of presentations. While other trial designs may be more realistic, such as $\mathrm{N}$ of 1 studies, tissue-based approaches, propensity matched analyses and adaptive design methods [55], Hellman originally endorsed that the ability to identify the oligometastatic state would profit from careful review of past experience, i.e. re- analysis of clinical data found in archival materials 
to arbitrate when, during the evolution of individual tumors, the oligometastatic state can be found.

\section{ACKNOWLEDGMENTS}

This work is financially supported by the NCI (grant no. U54CA143803, CA163124, CA093900 and CA143055 to K.J. Pienta).

\section{REFERENCES}

1. Weigelt B, Peterse JL van't Veer LJ. Breast cancer metastasis: markers and models. Nature reviews Cancer. 2005; 5:591-602.

2. Paget S. Distribution of secondary growths in cancer of the breast. Lancet. 1889; 1:571-573.

3. Halsted WS. The results of radical operations for the cure of carcinoma of the breast. Annals of Surgery. 1907; 46:1-19.

4. Tait CR, Waterworth A, Loncaster J, Horgan K, Dodwell D. The oligometastatic state in breast cancer: hypothesis or reality. Breast. 2005; 14:87-93.

5. Ewing J. (1928). MetastasisNeoplastics. (Philadelphia: Saunders) 1928; 77-89.

6. Keynes SGL. (1956). Carcinoma of the breast. Post-Graduate Committee FoM, Dalhousie University. Halifax, ed. The Nova Scotia Medical Bulletin) (St. Bartholomew's Hospital, London: 1956; 162-169.

7. Fisher B. Laboratory and clinical research in breast cancer - a personal adventure: the David A. Karnofsky memorial lecture. Cancer research. 1980; 40:3863-3874.

8. Lussier YA, Khodarev NN, Regan K, Corbin K, Li H, Ganai S, Khan SA, Gnerlich JL, Darga TE, Fan H, Karpenko O, Paty PB, Posner MC, Chmura SJ, Hellman S, Ferguson MK, et al. Oligo- and polymetastatic progression in lung metastasis(es) patients is associated with specific microRNAs. PloS one. 2012; 7:e50141.

9. Hellman S. Karnofsky Memorial Lecture. Natural history of small breast cancers. Journal of clinical oncology: official journal of the American Society of Clinical Oncology. 1994; 12:2229-2234.

10. Pienta KJ, Robertson BA, Coffey DS, Taichman RS. The cancer diaspora : Metastasis beyond the seed and soil hypothesis. Clinical cancer research: an official journal of the American Association for Cancer Research. 2013; 19:5849-5855.

11. Chambers AF, Groom AC, MacDonald IC. Dissemination and growth of cancer cells in metastatic sites. Nature reviews Cancer. 2002; 2:563-572.

12. Fidler IJ. The pathogenesis of cancer metastasis : the 'seed and soil' hypothesis revisited. Nature reviews Cancer. 2003; 3:453-458.

13. Corbin KS, Hellman S, Weichselbaum RR. Extracranial oligometastases : a subset of metastases curable with stereotactic radiotherapy. Journal of clinical oncology : official journal of the American Society of Clinical Oncology. 2013; 31:1384-1390.
14. Gupta GP, Massague J. Cancer metastasis : building a framework. Cell. 2006; 127:679-695.

15. Weichselbaum RR, Hellman S. Oligometastases revisited. Nature reviews Clinical oncology. 2011; 8:378-382.

16. Hanahan D, Weinberg RA. The hallmarks of cancer. Cell. $2000 ; 100: 57-70$.

17. Hanahan D, Weinberg RA. Hallmarks of cancer : the next generation. Cell. 2011; 144:646-674.

18. Warburg O. On the origin of cancer cells. Science. 1956; 123:309-314.

19. Shah RB, Mehra R, Chinnaiyan AM, Shen R, Ghosh D, Zhou M, Macvicar GR, Varambally S, Harwood J, Bismar TA, Kim R, Rubin MA, Pienta KJ. Androgenindependent prostate cancer is a heterogeneous group of diseases : lessons from a rapid autopsy program. Cancer research. 2004; 64:9209-9216.

20. Dhanasekaran SM, Barrette TR, Ghosh D, Shah R, Varambally S, Kurachi K, Pienta KJ, Rubin MA, Chinnaiyan AM. Delineation of prognostic biomarkers in prostate cancer. Nature. 2001; 412:822-826.

21. Varambally S, Dhanasekaran SM, Zhou M, Barrette TR, Kumar-Sinha C, Sanda MG, Ghosh D, Pienta KJ, Sewalt RG, Otte AP, Rubin MA, Chinnaiyan AM. The polycomb group protein EZH2 is involved in progression of prostate cancer. Nature. 2002; 419:624-629.

22. Ramaswamy S, Ross KN, Lander ES, Golub TR. A molecular signature of metastasis in primary solid tumors. Nature genetics. 2003; 33:49-54.

23. Haffner MC, Mosbruger T, Esopi DM, Fedor $\mathrm{H}$, Heaphy CM, Walker DA, Adejola N, Gurel M, Hicks J, Meeker AK, Halushka MK, Simons JW, Isaacs WB, De Marzo AM, Nelson WG, Yegnasubramanian S. Tracking the clonal origin of lethal prostate cancer. The Journal of clinical investigation. 2013; 123:4918-4922.

24. Gerlinger M, Rowan AJ, Horswell S, Larkin J, Endesfelder D, Gronroos E, Martinez P, Matthews N, Stewart A, Tarpey P, Varela I, Phillimore B, Begum S, McDonald NQ, Butler A, Jones D, et al. Intratumor heterogeneity and branched evolution revealed by multiregion sequencing. The New England journal of medicine. 2012; 366:883-892.

25. Drake JM, Graham NA, Lee JK, Stoyanova T, Faltermeier CM, Sud S, Titz B, Huang J, Pienta KJ, Graeber TG, Witte ON. Metastatic castration-resistant prostate cancer reveals intrapatient similarity and interpatient heterogeneity of therapeutic kinase targets. Proceedings of the National Academy of Sciences of the United States of America. 2013; 110:E4762-4769.

26. Fidler IJ, Kripke ML. Metastasis results from preexisting variant cells within a malignant tumor. Science. 1977; 197:893-895.

27. Chambers AF, Hill RP, Ling V. Tumor heterogeneity and stability of the metastatic phenotype of mouse KHT sarcoma cells. Cancer research. 1981; 41:1368-1372. 
28. Harris JF, Chambers AF, Hill RP, Ling V. Metastatic variants are generated spontaneously at a high rate in mouse KHT tumor. Proceedings of the National Academy of Sciences of the United States of America. 1982; 79:5547-5551.

29. Cillo C, Dick JE, Ling V, Hill RP. Generation of drugresistant variants in metastatic B16 mouse melanoma cell lines. Cancer research. 1987; 47:2604-2608.

30. Pantel K, Brakenhoff RH. Dissecting the metastatic cascade. Nature reviews Cancer. 2004; 4:448-456.

31. Kendal WS. Oligometastasis as a predictor for occult disease. Mathematical biosciences. 2014; 251:1-10.

32. Li Y, Tang ZY, Ye SL, Liu YK, Chen J, Xue Q, Chen J, Gao DM, Bao WH. Establishment of cell clones with different metastatic potential from the metastatic hepatocellular carcinoma cell line MHCC97. World journal of gastroenterology : WJG. 2001; 7:630-636.

33. Shindo-Okada N, Takeuchi K, Nagamachi Y. Establishment of cell lines with high- and low-metastatic potential from PC-14 human lung adenocarcinoma. Japanese journal of cancer research : Gann. 2001; 92:174-183.

34. Rastogi S, Gulia S, Bajpai J, Ghosh J, Gupta S. Oligometastatic breast cancer : A mini review. Indian journal of medical and paediatric oncology : official journal of Indian Society of Medical \& Paediatric Oncology. 2014; 35:203-206.

35. Kosari F, Parker AS, Kube DM, Lohse CM, Leibovich BC, Blute ML, Cheville JC, Vasmatzis G. Clear cell renal cell carcinoma : gene expression analyses identify a potential signature for tumor aggressiveness. Clinical cancer research: an official journal of the American Association for Cancer Research. 2005; 11:5128-5139.

36. Wuttig D, Baier B, Fuessel S, Meinhardt M, Herr A, Hoefling C, Toma M, Grimm MO, Meye A, Rolle A, Wirth MP. Gene signatures of pulmonary metastases of renal cell carcinoma reflect the disease-free interval and the number of metastases per patient. International journal of cancer Journal international du cancer. 2009; 125:474-482.

37. Jones RP, Stattner S, Sutton P, Dunne DF, McWhirter D, Fenwick SW, Malik HZ, Poston GJ. Controversies in the oncosurgical management of liver limited stage IV colorectal cancer. Surgical oncology. 2014; 23:53-60.

38. Vakiani E, Janakiraman M, Shen R, Sinha R, Zeng Z, Shia J, Cercek A, Kemeny N, D'Angelica M, Viale A, Heguy A, Paty P, Chan TA, Saltz LB, Weiser M, Solit DB. Comparative genomic analysis of primary versus metastatic colorectal carcinomas. Journal of clinical oncology : official journal of the American Society of Clinical Oncology. 2012; 30:2956-2962.

39. Stremitzer S, Stift J, Gruenberger B, Tamandl D, Aschacher T, Wolf B, Wrba F, Gruenberger T. KRAS status and outcome of liver resection after neoadjuvant chemotherapy including bevacizumab. The British journal of surgery. 2012; 99:1575-1582.
40. Bruin SC, de Ronde JJ, Wiering B, Braaf LM, de Wilt JH, Vincent AD, van Velthuysen ML, Ruers TJ, Wessels LF LJ. Selection of patients for hepatic surgery of colorectal cancer liver metastasis based on genomic aberrations. Annals of surgical oncology. 2013; 20:S560-569.

41. Cheng HH, Mitchell PS, Kroh EM, Dowell AE, Chery L, Siddiqui J, Nelson PS, Vessella RL, Knudsen BS, Chinnaiyan AM, Pienta KJ, Morrissey C, Tewari M. Circulating microRNA profiling identifies a subset of metastatic prostate cancer patients with evidence of cancerassociated hypoxia. PloS one. 2013; 8:e69239.

42. Huang F, Wu G, Yang K. Oligometastasis and oligo-recurrence : more than a mirage. Radiation oncology. 2014; 9:230.

43. Valastyan S, Weinberg RA. MicroRNAs : Crucial multitasking components in the complex circuitry of tumor metastasis. Cell cycle. 2009; 8:3506-3512.

44. Lussier YA, Xing HR, Salama JK, Khodarev NN, Huang Y, Zhang Q, Khan SA, Yang X, Hasselle MD, Darga TE, Malik R, Fan H, Perakis S, Filippo M, Corbin K, Lee Y, et al. MicroRNA expression characterizes oligometastasis(es). PloS one. 2011; 6:e28650.

45. Uppal A, Wightman SC, Mallon S, Oshima G, Pitroda SP, Zhang Q, Huang X, Darga TE, Huang L, Andrade J, Liu H, Ferguson MK, Greene GL, Posner MC, Hellman S, Khodarev NN, et al. 14q32-encoded microRNAs mediate an oligometastatic phenotype. Oncotarget. 2015; .

46. Hellman S, Weichselbaum RR. Oligometastases. Journal of clinical oncology : official journal of the American Society of Clinical Oncology. 1995; 13:8-10.

47. de Vin T, Engels B, Gevaert T, Storme G, De Ridder M. Stereotactic radiotherapy for oligometastatic cancer : a prognostic model for survival. Annals of oncology : official journal of the European Society for Medical Oncology / ESMO. 2014; 25:467-471.

48. Weichselbaum RR, Ishwaran H, Yoon $T$, Nuyten DS, Baker SW, Khodarev N, Su AW, Shaikh AY, Roach P, Kreike B, Roizman B, Bergh J, Pawitan Y, van de Vijver MJ, Minn AJ. An interferon-related gene signature for DNA damage resistance is a predictive marker for chemotherapy and radiation for breast cancer. Proceedings of the National Academy of Sciences of the United States of America. 2008; 105:18490-18495.

49. Macdermed DM, Weichselbaum RR, Salama JK. A rationale for the targeted treatment of oligometastases with radiotherapy. Journal of surgical oncology. 2008; 98:202-206.

50. Pagani O, Senkus E, Wood W, Colleoni M, Cufer T, Kyriakides S, Costa A, Winer EP, Cardoso F. International guidelines for management of metastatic breast cancer : can metastatic breast cancer be cured? Journal of the National Cancer Institute. 2010; 102:456-463.

51. Uppal A, Weichselbaum RR, Posner MC. Optimizing management of colorectal hepatic metastases : now and in the future. European journal of surgical oncology : the journal of the European Society of Surgical Oncology 
and the British Association of Surgical Oncology. 2014; 40:1033-1035.

52. Yao HH, Hong M, Corcoran NM, Siva S, Foroudi F. Advances in local and ablative treatment of oligometastasis in prostate cancer. Asia-Pacific journal of clinical oncology. 2014; 10:308-321.

53. Moreno A, Albiach C, Soria R, Vidal V, Gomez R, Antequera M. Oligometastases in prostate cancer : restaging stage IV cancers and new radiotherapy options. Radiation oncology. 2014; 9:258.

54. Reeves F, Murphy D, Evans C, Bowden P, Costello A. Targeted local therapy in oligometastatic prostate cancer : a promising potential opportunity after failed primary treatment. BJU international. 2014; .

55. Loh J, Davis ID, Martin JM, Siva S. Extracranial oligometastatic renal cell carcinoma : current management and future directions. Future oncology. 2014; 10:761-774.

56. Bhattasali O, Chen LN, Tong M, Lei S, Collins BT, Krishnan P, Kalhorn C, Lynch JH, Suy S, Dritschilo A, Dawson NA, Collins SP. Rationale for stereotactic body radiation therapy in treating patients with oligometastatic hormonenaive prostate cancer. Frontiers in oncology. 2013; 3:293.

57. Formenti SC, Demaria S. Combining radiotherapy and cancer immunotherapy : a paradigm shift. Journal of the National Cancer Institute. 2013; 105:256-265.

58. Matzinger $P$. The danger model : a renewed sense of self. Science. 2002; 296:301-305.

59. Lee Y, Auh SL, Wang Y, Burnette B, Wang Y, Meng Y, Beckett M, Sharma R, Chin R, Tu T, Weichselbaum RR, $\mathrm{Fu}$ YX. Therapeutic effects of ablative radiation on local tumor require $\mathrm{CD} 8+\mathrm{T}$ cells : changing strategies for cancer treatment. Blood. 2009; 114:589-595.

60. Lugade AA, Sorensen EW, Gerber SA, Moran JP, Frelinger JG, Lord EM. Radiation-induced IFN-gamma production within the tumor microenvironment influences antitumor immunity. Journal of immunology. 2008; 180:3132-3139.

61. Pawlik TM, Choti MA. Surgical therapy for colorectal metastases to the liver. Journal of gastrointestinal surgery : official journal of the Society for Surgery of the Alimentary Tract. 2007; 11:1057-1077.

62. Lam VW, Spiro C, Laurence JM, Johnston E, Hollands MJ, Pleass HC, Richardson AJ. A systematic review of clinical response and survival outcomes of downsizing systemic chemotherapy and rescue liver surgery in patients with initially unresectable colorectal liver metastases. Annals of surgical oncology. 2012; 19:1292-1301.

63. Deschamps F, Farouil G, Ternes N, Gaudin A, Hakime A, Tselikas L, Teriitehau C, Baudin E, Auperin A, de Baere T. Thermal ablation techniques : a curative treatment of bone metastases in selected patients? European radiology. 2014; 24:1971-1980.
64. Solomon LA, Munkarah AR, Vorugu VR, Deppe G, Adam B, Malone JM, Jr., Littrup PJ. Image-guided percutaneous cryotherapy for the management of gynecologic cancer metastases. Gynecologic oncology. 2008; 111:202-207.

65. Littrup PJ, Bang HJ, Currier BP, Goodrich DJ, Aoun HD, Heilbrun LK, Adam BA. Soft-tissue cryoablation in diffuse locations : feasibility and intermediate term outcomes. Journal of vascular and interventional radiology : JVIR. 2013; 24:1817-1825.

66. van Dongen JA, van Slooten EA. The surgical treatment of pulmonary metastases. Cancer treatment reviews. 1978; 5:29-48.

67. Institute NC. PDQ(R) Levels of Evidence for Adult and Pediatric Cancer Treatment Studies. (Bethesda, MD : National Cancer Institute).

68. Camacho LH, Kurzrock R, Cheung A, Barber DF, Gupta S, Madoff DC, Wallace MJ, Kim EE, Curley SA, Hortobagyi GN, Mavligit G. Pilot study of regional, hepatic intra-arterial paclitaxel in patients with breast carcinoma metastatic to the liver. Cancer. 2007; 109:2190-2196.

69. Bojko P, Welt A, Schleucher R, Borquez D, Scheulen ME, Vanhoefer U, Poettgen C, Stuschke M, Broelsch CE, Stamatis G, Wilke H, Seeber S, Harstrick A. High-dose chemotherapy with autologous stem cell transplantation in patients with oligometastatic breast cancer. Bone marrow transplantation. 2004; 34:637-643.

70. Milano MT, Zhang H, Metcalfe SK, Muhs AG, Okunieff P. Oligometastatic breast cancer treated with curative-intent stereotactic body radiation therapy. Breast cancer research and treatment. 2009; 115:601-608.

71. Kobayashi $T$, Ichiba $T$, Sakuyama $T$, Arakawa $Y$, Nagasaki E, Aiba K, Nogi H, Kawase K, Takeyama H, Toriumi Y, Uchida K, Kobayashi M, Kanehira C, Suzuki M, Ando N, Natori K, et al. Possible clinical cure of metastatic breast cancer : lessons from our 30-year experience with oligometastatic breast cancer patients and literature review. Breast cancer. 2012; 19:218-237.

72. Mimoto R, Kobayashi T, Imawari Y, Kamio M, Kato K, Nogi H, Toriumi Y, Hirooka S, Uchida K, Takeyama H. Clinical relevance and low tumor-initiating properties of oligometastatic breast cancer in pulmonary metastasectomy. Breast cancer research and treatment. 2014; 147:317-324.

73. VanderWalde A, Ye W, Frankel P, Asuncion D, Leong L, Luu T, Morgan R, Twardowski P, Koczywas M, Pezner R, Paz IB, Margolin K, Wong J, Doroshow JH, Forman S, Shibata S, et al. Long-term survival after high-dose chemotherapy followed by peripheral stem cell rescue for high-risk, locally advanced/inflammatory, and metastatic breast cancer. Biology of blood and marrow transplantation : journal of the American Society for Blood and Marrow Transplantation. 2012; 18:1273-1280.

74. Bourgier C, Khodari W, Vataire AL, Pessoa EL, Dunant A, Delaloge S, Uzan C, Balleyguier C, Mathieu MC, Marsiglia H, Arriagada R. Breast radiotherapy as part of 
loco-regional treatments in stage IV breast cancer patients with oligometastatic disease. Radiotherapy and oncology : journal of the European Society for Therapeutic Radiology and Oncology. 2010; 96:199-203.

75. Nieto Y, Nawaz S, Jones RB, Shpall EJ, Cagnoni PJ, McSweeney PA, Baron A, Razook C, Matthes S, Bearman SI. Prognostic model for relapse after high-dose chemotherapy with autologous stem-cell transplantation for stage IV oligometastatic breast cancer. Journal of clinical oncology : official journal of the American Society of Clinical Oncology. 2002; 20:707-718.

76. Ashworth A, Rodrigues G, Boldt G, Palma D. Is there an oligometastatic state in non-small cell lung cancer? A systematic review of the literature. Lung cancer. 2013; 82:197-203.

77. Lanuti M, Sequist LV, Sharma A, Mino-Kenudson M. Case records of the Massachusetts General Hospital. Case 31-2011. A 55-year-old man with oligometastatic lung cancer. The New England journal of medicine. 2011; 365:1426-1435.

78. Grossi F, Kubota K, Cappuzzo F, de Marinis F, Gridelli C, Aita M, Douillard JY. Future scenarios for the treatment of advanced non-small cell lung cancer : focus on taxanecontaining regimens. The oncologist. 2010; 15:1102-1112.

79. Strong VE, D'Angelica M, Tang L, Prete F, Gonen M, Coit D, Touijer KA, Fong Y, Brennan MF. Laparoscopic adrenalectomy for isolated adrenal metastasis. Annals of surgical oncology. 2007; 14:3392-3400.

80. Collen C, Christian N, Schallier D, Meysman M, Duchateau M, Storme G, De Ridder M. Phase II study of stereotactic body radiotherapy to primary tumor and metastatic locations in oligometastatic nonsmall-cell lung cancer patients. Annals of oncology : official journal of the European Society for Medical Oncology / ESMO. 2014; 25:1954-1959.

81. De Ruysscher D, Wanders R, van Baardwijk A, Dingemans AM, Reymen B, Houben R, Bootsma G, Pitz C, van Eijsden L, Geraedts W, Baumert BG, Lambin P. Radical treatment of non-small-cell lung cancer patients with synchronous oligometastases : long-term results of a prospective phase II trial (Nct01282450). Journal of thoracic oncology : official publication of the International Association for the Study of Lung Cancer. 2012; 7:1547-1555.

82. Nieder C, Tollali T, Reigstad A, Pawinski A, Haukland E, Dalhaug A. Oligometastatic non-small cell lung cancer : a significant entity outside of specialized cancer centers? Medical principles and practice : international journal of the Kuwait University, Health Science Centre. 2014; 23:526-531.

83. Khan AJ, Mehta PS, Zusag TW, Bonomi PD, Penfield Faber L, Shott S, Abrams RA. Long term disease-free survival resulting from combined modality management of patients presenting with oligometastatic, non-small cell lung carcinoma (NSCLC). Radiotherapy and oncology : journal of the European Society for Therapeutic Radiology and Oncology. 2006; 81:163-167.
84. Ashworth AB, Senan S, Palma DA, Riquet M, Ahn YC, Ricardi U, Congedo MT, Gomez DR, Wright GM, Melloni G, Milano MT, Sole CV, De Pas TM, Carter DL, Warner AJ, Rodrigues GB. An individual patient data metaanalysis of outcomes and prognostic factors after treatment of oligometastatic non-small-cell lung cancer. Clinical lung cancer. 2014; 15:346-355.

85. Lopez Guerra JL, Gomez D, Zhuang Y, Hong DS, Heymach JV, Swisher SG, Lin SH, Komaki R, Cox JD, Liao Z. Prognostic impact of radiation therapy to the primary tumor in patients with non-small cell lung cancer and oligometastasis at diagnosis. International journal of radiation oncology, biology, physics. 2012; 84:e61-67.

86. Collaud S, Stahel R, Inci I, Hillinger S, Schneiter D, Kestenholz P, Weder W. Survival of patients treated surgically for synchronous single-organ metastatic NSCLC and advanced pathologic TN stage. Lung cancer. 2012; 78:234-238.

87. Congedo MT, Cesario A, Lococo F, De Waure C, Apolone G, Meacci E, Cavuto S, Granone P. Surgery for oligometastatic non-small cell lung cancer : long-term results from a single center experience. The Journal of thoracic and cardiovascular surgery. 2012; 144:444-452.

88. Hasselle MD, Haraf DJ, Rusthoven KE, Golden DW, Salgia R, Villaflor VM, Shah N, Hoffman PC, Chmura SJ, Connell PP, Vokes EE, Weichselbaum RR, Salama JK. Hypofractionated image-guided radiation therapy for patients with limited volume metastatic non-small cell lung cancer. Journal of thoracic oncology : official publication of the International Association for the Study of Lung Cancer. 2012; 7:376-381.

89. Griffioen GH, Toguri D, Dahele M, Warner A, de Haan PF, Rodrigues GB, Slotman BJ, Yaremko BP, Senan S, Palma DA. Radical treatment of synchronous oligometastatic non-small cell lung carcinoma (NSCLC) : patient outcomes and prognostic factors. Lung cancer. 2013; 82:95-102.

90. Yano T, Okamoto T, Haro A, Fukuyama S, Yoshida T, Kohno M, Maehara Y. Local treatment of oligometastatic recurrence in patients with resected non-small cell lung cancer. Lung cancer. 2013; 82:431-435.

91. Yu HA, Sima CS, Huang J, Solomon SB, Rimner A, Paik P, Pietanza MC, Azzoli CG, Rizvi NA, Krug LM, Miller VA, Kris MG, Riely GJ. Local therapy with continued EGFR tyrosine kinase inhibitor therapy as a treatment strategy in EGFR-mutant advanced lung cancers that have developed acquired resistance to EGFR tyrosine kinase inhibitors. Journal of thoracic oncology : official publication of the International Association for the Study of Lung Cancer. 2013; 8:346-351.

92. Gray PJ, Mak RH, Yeap BY, Cryer SK, Pinnell NE, Christianson LW, Sher DJ, Arvold ND, Baldini EH, Chen AB, Kozono DE, Swanson SJ, Jackman DM, Alexander BM. Aggressive therapy for patients with nonsmall cell lung carcinoma and synchronous brain-only 
oligometastatic disease is associated with long-term survival. Lung cancer. 2014; 85:239-244.

93. Cheufou DH, Welter S, Chalvatzoulis E, Christof D, Theegarten D, Stamatis G. Surgery of primary lung cancer with oligometastatic $\mathrm{m} 1 \mathrm{~b}$ synchronous single brain metastasis : analysis of 37 cases. The Thoracic and cardiovascular surgeon. 2014; 62:612-615.

94. Parikh RB, Cronin AM, Kozono DE, Oxnard GR, Mak RH, Jackman DM, Lo PC, Baldini EH, Johnson BE, Chen AB. Definitive primary therapy in patients presenting with oligometastatic non-small cell lung cancer. International journal of radiation oncology, biology, physics. 2014; 89:880-887.

95. Sheu T, Heymach JV, Swisher SG, Rao G, Weinberg JS, Mehran R, McAleer MF, Liao Z, Aloia TA, Gomez DR. Propensity score-matched analysis of comprehensive local therapy for oligometastatic non-small cell lung cancer that did not progress after front-line chemotherapy. International journal of radiation oncology, biology, physics. 2014; 90:850-857.

96. Tonnies M, Pfannschmidt J, Bauer TT, Kollmeier J, Tonnies S, Kaiser D. Metastasectomy for synchronous solitary non-small cell lung cancer metastases. The Annals of thoracic surgery. 2014; 98:249-256.

97. Ouyang WW, Su SF, Ma Z, Hu YX, Lu B, Li QS, Geng YC, Li HQ. Prognosis of non-small cell lung cancer patients with bone oligometastases treated concurrently with thoracic three-dimensional radiotherapy and chemotherapy. Radiation oncology. 2014; 9:147.

98. Endo C, Hasumi T, Matsumura Y, Sato N, Deguchi H, Oizumi H, Sagawa M, Tsushima T, Takahashi S, Shibuya J, Hirose M, Kondo T. A prospective study of surgical procedures for patients with oligometastatic non-small cell lung cancer. The Annals of thoracic surgery. 2014; 98:258-264.

99. Essner R, Lee JH, Wanek LA, Itakura H and Morton DL. Contemporary surgical treatment of advanced-stage melanoma. Archives of surgery. 2004; 139:961-966; discussion 966-967.

100. Knisely JP, Yu JB, Flanigan J, Sznol M, Kluger HM, Chiang VL. Radiosurgery for melanoma brain metastases in the ipilimumab era and the possibility of longer survival. Journal of neurosurgery. 2012; 117:227-233.

101. Engel J, Bastian PJ, Baur H, Beer V, Chaussy C, Gschwend JE, Oberneder R, Rothenberger KH, Stief CG, Holzel D. Survival benefit of radical prostatectomy in lymph node-positive patients with prostate cancer. European urology. 2010; 57:754-761.

102. Jemal A, Siegel R, Ward E, Hao Y, Xu J and Thun MJ. Cancer statistics, 2009. CA: a cancer journal for clinicians. 2009; 59:225-249.

103. Timmerman RD, Bizekis CS, Pass HI, Fong Y, Dupuy DE, Dawson LA and Lu D. Local surgical, ablative, and radiation treatment of metastases. CA : a cancer journal for clinicians. 2009; 59:145-170.

104. Engels B, Gevaert T, Everaert H, De Coninck P, Sermeus A, Christian N, Storme G, Verellen D, De Ridder M. Phase II study of helical tomotherapy in the multidisciplinary treatment of oligometastatic colorectal cancer. Radiation oncology. 2012; 7:34.

105. Hughes KS, Simon R, Songhorabodi S, Adson MA, Ilstrup DM, Fortner JG, Maclean BJ, Foster JH, Daly JM, Fitzherbert D, et al. Resection of the liver for colorectal carcinoma metastases : a multi-institutional study of patterns of recurrence. Surgery. 1986; 100:278-284.

106. Nordlinger B, Guiguet M, Vaillant JC, Balladur P, Boudjema K, Bachellier P, Jaeck D. Surgical resection of colorectal carcinoma metastases to the liver. A prognostic scoring system to improve case selection, based on 1568 patients. Association Francaise de Chirurgie. Cancer. 1996; 77:1254-1262.

107. Fong Y, Fortner J, Sun RL, Brennan MF, Blumgart LH. Clinical score for predicting recurrence after hepatic resection for metastatic colorectal cancer : analysis of 1001 consecutive cases. Ann Surg. 1999; 230:309-318. discussion 318-321.

108. Pawlik TM, Scoggins CR, Zorzi D, Abdalla EK, Andres A, Eng C, Curley SA, Loyer EM, Muratore A, Mentha G, Capussotti L, Vauthey JN. Effect of surgical margin status on survival and site of recurrence after hepatic resection for colorectal metastases. Ann Surg. 2005; 241:715-722. discussion 722-714.

109. Tomlinson JS, Jarnagin WR, DeMatteo RP, Fong Y, Kornprat P, Gonen M, Kemeny N, Brennan MF, Blumgart LH, D’Angelica M. Actual 10-year survival after resection of colorectal liver metastases defines cure. Journal of clinical oncology : official journal of the American Society of Clinical Oncology. 2007; 25:4575-4580.

110. Dellas K, Reese T, Richter M, Arnold D, Dunst J. Concurrent chemoradiation of metastases with capecitabine and oxaliplatin and 3D-CRT in patients with oligometastatic colorectal cancer : results of a phase I study. Radiation oncology. 2012; 7:83.

111. Comito T, Cozzi L, Clerici E, Campisi MC, Liardo RL, Navarria P, Ascolese A, Tozzi A, Iftode C, De Rose F, Villa E, Personeni N, Rimassa L, Santoro A, Fogliata A, Mancosu P, et al. Stereotactic Ablative Radiotherapy (SABR) in inoperable oligometastatic disease from colorectal cancer : a safe and effective approach. BMC cancer. 2014; 14:619.

112. Salah S, Watanabe K, Welter S, Park JS, Park JW, Zabaleta J, Ardissone F, Kim J, Riquet M, Nojiri K, Gisabella M, Kim SY, Tanaka K, Al-Haj Ali B. Colorectal cancer pulmonary oligometastases : pooled analysis and construction of a clinical lung metastasectomy prognostic model. Annals of oncology : official journal of the 
European Society for Medical Oncology / ESMO. 2012; 23:2649-2655.

113. Van den Begin R, Engels B, Gevaert T, Duchateau M, Tournel K, Verellen D, Storme G, De Ridder M. Impact of inadequate respiratory motion management in SBRT for oligometastatic colorectal cancer. Radiotherapy and oncology : journal of the European Society for Therapeutic Radiology and Oncology. 2014; 113:235-239.

114. Filippi AR, Badellino S, Ceccarelli M, Guarneri A, Franco P, Monagheddu C, Spadi R, Ragona R, Racca P, Ricardi U. Stereotactic Ablative Radiation Therapy as First Local Therapy for Lung Oligometastases From Colorectal Cancer : A Single-Institution Cohort Study. International journal of radiation oncology, biology, physics. 2014.

115. Salah S, Watanabe K, Park JS, Addasi A, Park JW, Zabaleta J, Ardissone F, Kim J, Riquet M, Nojiri K, Gisabella M, Kim SY, Tanaka K. Repeated resection of colorectal cancer pulmonary oligometastases : pooled analysis and prognostic assessment. Annals of surgical oncology. 2013; 20:1955-1961.

116. Bae SH, Kim MS, Cho CK, Kang JK, Kang HJ, Kim YH, Shin US, Moon SM, Lee DH. High dose stereotactic body radiotherapy using three fractions for colorectal oligometastases. Journal of surgical oncology. 2012; 106:138-143.

117. Kang JK, Kim MS, Kim JH, Yoo SY, Cho CK, Yang KM, Yoo HJ, Seo YS, Lee DH, Kang HJ, Kim YH, Shin US. Oligometastases confined one organ from colorectal cancer treated by SBRT. Clinical \& experimental metastasis. 2010; 27:273-278.

118. Hohenberger P, Kasper B, Ahrar K. Surgical management and minimally invasive approaches for the treatment of metastatic sarcoma. American Society of Clinical Oncology educational book / ASCO American Society of Clinical Oncology Meeting. 2013; :457-464.

119. Falk AT, Moureau-Zabotto L, Ouali M, Penel N, Italiano A, Bay JO, Olivier T, Sunyach MP, BoudouRoquette P, Salas S, Le Maignan C, Ducassou A, Isambert N, Kalbacher E, Pan C, Saada E, et al. Effect on survival of local ablative treatment of metastases from sarcomas : a study of the French sarcoma group. Clinical oncology. 2015; 27:48-55.

120. Rhomberg W, Eiter H, Schmid F, Saely CH. Combined vindesine and razoxane shows antimetastatic activity in advanced soft tissue sarcomas. Clinical \& experimental metastasis. 2008; 25:75-80.

121. Motzer RJ, Bander NH, Nanus DM. Renal-cell carcinoma. The New England journal of medicine. 1996; 335:865-875.

122. Ranck MC, Golden DW, Corbin KS, Hasselle MD, Liauw SL, Stadler WM, Hahn OM, Weichselbaum RR, Salama JK. Stereotactic body radiotherapy for the treatment of oligometastatic renal cell carcinoma. American journal of clinical oncology. 2013; 36:589-595.
123. Thibault I, Al-Omair A, Masucci GL, Masson-Cote L, Lochray F, Korol R, Cheng L, Xu W, Yee A, Fehlings MG, Bjarnason GA, Sahgal A. Spine stereotactic body radiotherapy for renal cell cancer spinal metastases : analysis of outcomes and risk of vertebral compression fracture. Journal of neurosurgery Spine. 2014; 21:711-718.

124. Bang HJ, Littrup PJ, Goodrich DJ, Currier BP, Aoun HD, Heilbrun LK, Vaishampayan U, Adam B, Goodman AC. Percutaneous cryoablation of metastatic renal cell carcinoma for local tumor control : feasibility, outcomes, and estimated cost-effectiveness for palliation. Journal of vascular and interventional radiology : JVIR. 2012; 23:770-777.

125. Mickisch GH, Garin A, van Poppel H, de Prijck L, Sylvester R. Radical nephrectomy plus interferonalfa-based immunotherapy compared with interferon alfa alone in metastatic renal-cell carcinoma : a randomised trial. Lancet. 2001; 358:966-970.

126. Flanigan RC, Salmon SE, Blumenstein BA, Bearman SI, Roy V, McGrath PC, Caton JR, Jr, Munshi N, Crawford ED. Nephrectomy followed by interferon alfa-2b compared with interferon alfa-2b alone for metastatic renalcell cancer. The New England journal of medicine. 2001; 345:1655-1659.

127. Widmark A, Klepp O, Solberg A, Damber JE, Angelsen A, Fransson P, Lund JA, Tasdemir I, Hoyer M, Wiklund F, Fossa SD. Endocrine treatment, with or without radiotherapy, in locally advanced prostate cancer (SPCG-7/ SFUO-3) : an open randomised phase III trial. Lancet. 2009; 373:301-308.

128. Vickers A, Bennette C, Steineck G, Adami HO, Johansson JE, Bill-Axelson A, Palmgren J, Garmo H, Holmberg L. Individualized estimation of the benefit of radical prostatectomy from the Scandinavian Prostate Cancer Group randomized trial. European urology. 2012; 62:204-209.

129. Zelefsky MJ, Eastham JA, Cronin AM, Fuks Z, Zhang Z, Yamada Y, Vickers A, Scardino PT. Metastasis after radical prostatectomy or external beam radiotherapy for patients with clinically localized prostate cancer : a comparison of clinical cohorts adjusted for case mix. Journal of clinical oncology : official journal of the American Society of Clinical Oncology. 2010; 28:1508-1513.

130. Pierorazio PM, Lin BM, Mullins JK, Hyndman ME, Schaeffer EM, Han M, Partin AW, Pavlovich CP. Preoperative characteristics of men with unfavorable highGleason prostate cancer at radical prostatectomy. Urologic oncology. 2013; 31:589-594.

131. Shao YH, Kim S, Moore DF, Shih W, Lin Y, Stein M, Kim IY, Lu-Yao GL. Cancer-specific survival after metastasis following primary radical prostatectomy compared with radiation therapy in prostate cancer patients : results of a population-based, propensity score-matched analysis. European urology. 2014; 65:693-700. 
132. Cadeddu JA, Partin AW, Epstein JI, Walsh PC. Stage D1 (T1-3, N1-3, M0) prostate cancer : a case-controlled comparison of conservative treatment versus radical prostatectomy. Urology. 1997; 50:251-255.

133. Ost P, Bossi A, Decaestecker K, De Meerleer G, Giannarini G, Karnes RJ, Roach M, 3rd, Briganti A. Metastasis-directed Therapy of Regional and Distant Recurrences After Curative Treatment of Prostate Cancer : A Systematic Review of the Literature. European urology. 2014.

134. Culp SH, Schellhammer PF, Williams MB. Might men diagnosed with metastatic prostate cancer benefit from definitive treatment of the primary tumor? A SEER-based study. European urology. 2014; 65:1058-1066.

135. Zapatero A, Guerrero, A., Maldonado, J. , Alvarez, A., San Segundo, C.G., Rodriguez, M.C., Macias, V., PedroOlive, A., Casas, F., Boladeras, A., de Vidales, C.M., de la Torre, M.V., Calvo, F.A. Randomized Phase III Trial of Adjuvant Androgen Deprivation in Combination with High-dose Conformal Radiotherapy in Intermediate and High Risk Localized Prostate Cancer. Journal of clinical oncology : official journal of the American Society of Clinical Oncology. 2014; 32:52.

136. McKay RR, Choueiri TK, Taplin ME. Rationale for and review of neoadjuvant therapy prior to radical prostatectomy for patients with high-risk prostate cancer. Drugs. $2013 ; 73: 1417-1430$.

137. James ND, Spears MR, Clarke NW, Dearnaley DP, De Bono JS, Gale J, Hetherington J, Hoskin PJ, Jones RJ, Laing R, Lester JF, McLaren D, Parker CC, Parmar MK, Ritchie AW, Russell JM, et al. Survival with Newly Diagnosed Metastatic Prostate Cancer in the "Docetaxel Era" : Data from 917 Patients in the Control Arm of the STAMPEDE Trial (MRC PR08, CRUK/06/019). European urology. 2014.

138. Maximum androgen blockade in advanced prostate cancer. an overview of the randomised trials. Prostate Cancer Trialists' Collaborative Group. Lancet. 2000; 355:1491-1498

139. Heidenreich A, Pfister D, Brehmer B, Porres D. [Cytoreductive radical prostatectomy for prostate cancer with minimal osseous metastases : Results of a first feasibility and case control study]. Der Urologe Ausg A. 2015; $54: 14-21$.

140. Abdollah F, Karnes RJ, Suardi N, Cozzarini C, Gandaglia G, Fossati N, Vizziello D, Sun M, Karakiewicz PI, Menon M, Montorsi F, Briganti A. Impact of adjuvant radiotherapy on survival of patients with node-positive prostate cancer. Journal of clinical oncology : official journal of the American Society of Clinical Oncology. 2014; 32:3939-3947.

141. Schick U, Jorcano S, Nouet P, Rouzaud M, Vees H, Zilli T, Ratib O, Weber DC, Miralbell R. Androgen deprivation and high-dose radiotherapy for oligometastatic prostate cancer patients with less than five regional and/or distant metastases. Acta oncologica. 2013; 52:1622-1628.

142. Ost P, Decaestecker K, Lambert B, Fonteyne V, Delrue L, Lumen N, Ameye F, De Meerleer G. Prognostic factors influencing prostate cancer-specific survival in non-castrate patients with metastatic prostate cancer. The Prostate. 2014; 74:297-305.

143. Ponti E, Ingrosso G, Carosi A, Di Murro L, Lancia A, Pietrasanta F, Santoni R. Salvage Stereotactic Body Radiotherapy for Patients With Prostate Cancer With Isolated Lymph Node Metastasis : A Single-Center Experience. Clinical genitourinary cancer. 2014.

144. Decaestecker K, De Meerleer G, Lambert B, Delrue L, Fonteyne V, Claeys T, De Vos F, Huysse W, Hautekiet A, Maes G, Ost P. Repeated stereotactic body radiotherapy for oligometastatic prostate cancer recurrence. Radiation oncology. 2014; 9:135.

145. Ahmed KA, Barney BM, Davis BJ, Park SS, Kwon ED, Olivier KR. Stereotactic body radiation therapy in the treatment of oligometastatic prostate cancer. Frontiers in oncology. 2012; 2:215.

146. Berkovic P, De Meerleer G, Delrue L, Lambert B, Fonteyne V, Lumen N, Decaestecker K, Villeirs G, Vuye P, Ost P. Salvage stereotactic body radiotherapy for patients with limited prostate cancer metastases : deferring androgen deprivation therapy. Clinical genitourinary cancer. 2013; 11:27-32.

147. Jereczek-Fossa BA, Piperno G, Ronchi S, Catalano G, Fodor C, Cambria R, Fossati Ing P, Gherardi F, Alterio D, Zerini D, Garibaldi C, Baroni G, De Cobelli O, Orecchia R. Linac-based stereotactic body radiotherapy for oligometastatic patients with single abdominal lymph node recurrent cancer. American journal of clinical oncology. 2014; 37:227-233.

148. Tabata K, Niibe Y, Satoh T, Tsumura H, Ikeda M, Minamida S, Fujita T, Ishii D, Iwamura M, Hayakawa K, Baba S. Radiotherapy for oligometastases and oligo-recurrence of bone in prostate cancer. Pulmonary medicine. 2012; 2012:541656.

149. Singh D, Yi WS, Brasacchio RA, Muhs AG, Smudzin T, Williams JP, Messing E, Okunieff P. Is there a favorable subset of patients with prostate cancer who develop oligometastases? International journal of radiation oncology, biology, physics. 2004; 58:3-10.

150. Decaestecker K, De Meerleer G, Ameye F, Fonteyne V, Lambert B, Joniau S, Delrue L, Billiet I, Duthoy W, Junius S, Huysse W, Lumen N, Ost P. Surveillance or metastasis-directed Therapy for OligoMetastatic Prostate cancer recurrence (STOMP) : study protocol for a randomized phase II trial. BMC cancer. 2014; 14:671.

151. Attard G, Sydes MR, Mason MD, Clarke NW, Aebersold D, de Bono JS, Dearnaley DP, Parker CC, Ritchie AW, Russell JM, Thalmann G, Cassoly E, Millman R, Matheson D, Schiavone F, Spears MR, et al. Combining Enzalutamide with Abiraterone, Prednisone, and Androgen 
Deprivation Therapy in the STAMPEDE Trial. European urology. 2014.

152. Health USNIo. In: ClinicalTrials.gov [Internet]. (Bethesda (MD) : National Library of Medicine (US). 2000-.

153. Hospital CUoHKPYNE. ATOM_local Ablative Therapy. In: ClinicalTrials.gov [Internet]. (Bethesda (MD) : National Library of Medicine (US) 2000-.

154. Institute RPCINC. Stereotactic Body Radiation Therapy After Surgery in Treating Patients With Stage III-IV Nonsmall Cell Lung Cancer. In: ClinicalTrials.gov [Internet]. (Bethesda (MD) : National Library of Medicine (US) 2000-.

155. University CCCoWF. Stereotactic Body Radiation Therapy (SBRT) in Metastatic Non-small Cell Lung Cancer. In: ClinicalTrials.gov [Internet]. (Bethesda (MD) : National Library of Medicine (US) 2000-.

156. Institute NCCTGNC. Radiation Therapy or Observation After Chemotherapy in Treating Patients With Stage IV Non-Small Cell Lung Cancer. In: ClinicalTrials.gov [Internet]. (Bethesda (MD) : National Library of Medicine (US) 2000-.

157. Oncology MR. Concurrent and Non-concurrent Chemo-radiotherapy or Radiotherapy Alone for Patients With Oligo-metastatic Stage IV Non-small Cell Lung Cancer (NSCLC). In: ClinicalTrials.gov [Internet]. Bethesda (MD) : National Library of Medicine (US)2000-.

158. Institute SCHaR. The Optimal Intervention Time of Radiotherapy for Oligometastatic Stage IV Non-small Cell Lung Cancer $\square$ NSCLC) (OITROLC). In: ClinicalTrials. gov [Internet]. (Bethesda (MD) : National Library of Medicine (US) 2000-.

159. Foundation WJM. The Value of Radiotherapy in the Oligometastatic Non-squamous Non-small Cell Lung Cancer With Clinical Benefits From Erlotinib as Secondline Treatment (ROLE). In: ClinicalTrials.gov [Internet]. (Bethesda (MD) : National Library of Medicine (US) 2000-.

160. Pennsylvania ACCotUo. Phase II Study of Pembrolizumab After Curative Intent Treatment for Oligometastatic NonSmall Cell Lung Cancer. In: ClinicalTrials.gov [Internet]. (Bethesda (MD) : National Library of Medicine (US) 2000-.

161. Center MDAC. Oligometastatic Disease. In: ClinicalTrials. gov [Internet]. (Bethesda (MD) : National Library of Medicine (US) 2000-.

162. Florida Uo. Radiotherapy for Oligometastatic Prostate Cancer. In: ClinicalTrials.gov [Internet]. (Bethesda (MD) : National Library of Medicine (US) 2000-.

163. Clinic M. Monitoring Anti-Prostate Cancer Immunity Following Stereotactic Body Radiotherapy (SBRT). In: ClinicalTrials.gov [Internet]. (Bethesda (MD) : National Library of Medicine (US) 2000-.
164. Castellon GdICeORHPd. Phase II Study of SBRT as Treatment for Oligometastases in Prostate Cancer. In: ClinicalTrials.gov [Internet]. (Bethesda (MD) : National Library of Medicine (US) 2000-.

165. Dresden TU. Percutaneous High-dose Radiotherapy in Patients With Oligometastases of Prostate Carcinoma (Oli-P). In: ClinicalTrials.gov [Internet]. (Bethesda (MD) : National Library of Medicine (US) 2000-.

166. l'Ouest ICd. Salvage Radiotherapy Combined With Hormonotherapy in Oligometastatic Pelvic Node Relapses of Prostate Cancer (OLIGOPELVIS). In: ClinicalTrials. gov [Internet]. (Bethesda (MD) : National Library of Medicine (US) 2000-.

167. Institute CoHMCNC. Intensity-Modulated Radiation Therapy in Treating Patients Undergoing Androgen Deprivation Therapy for Metastatic Prostate Cancer. In: ClinicalTrials.gov [Internet]. (Bethesda (MD) : National Library of Medicine (US) 2000-.

168. Center CoHM. Intensity-Modulated Radiation Therapy in Treating Patients Undergoing Androgen Deprivation Therapy for Metastatic Prostate Cancer. In: ClinicalTrials. gov [Internet]. (Bethesda (MD) : National Library of Medicine (US) 2000-.

169. University Hospital G. Non-systemic Treatment for Patients With Low-volume Metastatic Prostate Cancer. In: ClinicalTrials.gov [Internet]. (Bethesda (MD) : National Library of Medicine (US) 2000-.

170. Center MS-KC. Combining Ipilimumab, Degarelix, and Radical Prostatectomy in Men With Newly Diagnosed Metastatic Castration Sensitive Prostate Cancer or Ipilimumab and Degarelix in Men With Biochemically Recurrent Castration Sensitive Prostate Cancer After Radical Prostatectomy. In: ClinicalTrials.gov [Internet]. (Bethesda (MD) : National Library of Medicine (US) 2000-.

171. Institute TNC. High Dose Chemotherapy in Oligometastatic Homologous Recombination Deficient Breast Cancer. In: ClinicalTrials.gov [Internet]. (Bethesda (MD) : National Library of Medicine (US) 2000-.

172. Peter MacCallum Cancer Centre A. A Pilot Study of Stereotactic Ablation for Oligometastatic Breast Neoplasia in Combination With the Anti-PD-1 Antibody MK-3475. (BOSTON II). In: ClinicalTrials.gov [Internet]. (Bethesda (MD) : National Library of Medicine (US) 2000-.

173. Gustave Roussy CC Grand Paris. Trial of Superiority of Stereotactic Body Radiation Therapy in Patients With Breast Cancer (STEREO-SEIN). In: ClinicalTrials.gov [Internet]. (Bethesda (MD) : National Library of Medicine (US) 2000-.

174. Institute CoHMCNC. Combination Chemotherapy With or Without Trastuzumab Followed By an Autologous Stem Cell Transplant and Radiation Therapy in Treating Patients With Stage III or Stage IV Breast Cancer. In ClinicalTrials.gov [Internet]. (Bethesda (MD) : National Library of Medicine (US) 2000-. 
175. Center ULCC. Defining the HER2 Positive $(+)$ Breast Cancer Kinome Response to Trastuzumab, Pertuzumab, Combination Trastuzumab +Pertuzumab, or Combination Trastuzumab + Lapatinib. In: ClinicalTrials.gov [Internet]. (Bethesda (MD) : National Library of Medicine (US) 2000-.

176. Institute UoCNC. Hypofractionated Image Guided Radiation Therapy in Treating Patients With Stage IV Breast Cancer. In: ClinicalTrials.gov [Internet]. (Bethesda (MD) : National Library of Medicine (US) 2000-.

177. Nevada CCCo. Concurrent Ipilimumab and Stereotactic Ablative Radiation Therapy (SART) for Oligometastatic But Unresectable Melanoma. In: ClinicalTrials.gov [Internet]. (Bethesda (MD) : National Library of Medicine (US) 2000-.

178. Center OSUCC. Stereotactic Radiation Therapy and Ipilimumab in Treating Patients With Metastatic Melanoma. In: ClinicalTrials.gov [Internet]. (Bethesda (MD) : National Library of Medicine (US) 2000-.

179. Center MDAC. Combi-Neo Study for Stage IV Melanoma. In: ClinicalTrials.gov [Internet]. (Bethesda (MD) : National Library of Medicine (US) 2000-.

180. Center SKCC. Stereotactic Body Radiation With Nelfinavir for Oligometastases. In: ClinicalTrials.gov [Internet]. (Bethesda (MD) : National Library of Medicine (US) 2000-.

181. Dwight Heron UoP. Radiosurgery for Patients With Oligometastatic Disease at Initial Presentation. In: ClinicalTrials.gov [Internet]. (Bethesda (MD) : National Library of Medicine (US) 2000-.

182. Dwight Heron UoP. Radiosurgery for Patients Recurrent Oligometastatic Disease. In: ClinicalTrials.gov [Internet]. (Bethesda (MD) : National Library of Medicine (US) 2000-.

183. Brussel UZ. Non-interventional Observational Study of Stereotactic Body Radiotherapy (SBRT) for Oligometastatic Cancer. In: ClinicalTrials.gov [Internet]. (Bethesda (MD) : National Library of Medicine (US) 2000-.

184. University Health Network TPMH, Canada. 5 Fraction Stereotactic Body Radiation Therapy for Oligometastases Regimen, for Extra-Cranial Oligometastases. In: ClinicalTrials.gov [Internet]. (Bethesda (MD) : National Library of Medicine (US) 2000-.

185. Center UoTSM. Prospective Clinical Registry for Oligometastic Disease, Consolidation Therapy, Debulking Prior to Chemotherapy, or Re-Irradiation. In: ClinicalTrials.gov [Internet]. (Bethesda (MD) : National Library of Medicine (US) 2000-.

186. Centers RMC. Definitive Therapy for Oligometastatic Solid Malignancies. In: ClinicalTrials.gov [Internet]. (Bethesda (MD) : National Library of Medicine (US) $2000-$.
187. Medicine MSSo. Sutent and Radiation as Treatment for Limited Extent Metastatic Cancer. In: ClinicalTrials.gov [Internet]. (Bethesda (MD) : National Library of Medicine (US) 2000-.

188. Oncology MR. Assessment of Toxicity of the Immunocytokine L19-IL2 Administered Directly After the Course of Stereotactic Ablative Body Radiotherapy in Patients Suffering From Oligometastatic Solid Tumour. In: ClinicalTrials.gov [Internet]. (Bethesda (MD) : National Library of Medicine (US) 2000-.

189. (TROG) T-TROG. A Randomized Phase II Study of Stereotactic Ablative Body Radiotherapy for Metastases to the Lung, (TROG 13.01 SAFRON II). In: ClinicalTrials. gov [Internet]. (Bethesda (MD) : National Library of Medicine (US) 2000-.

190. Lawson Health Research Institute; London Regional Cancer Program CVUoA. Stereotactic Ablative Radiotherapy for Comprehensive Treatment of Oligometastatic Tumors (SABR-COMET). In: ClinicalTrials.gov [Internet]. (Bethesda (MD) : National Library of Medicine (US) 2000-.

191. Medicine WUSo. Adaptive MRI-Guided SBRT for Unresectable Primary or Oligometastatic Central Thorax and Abdominal Malignancies. In: ClinicalTrials.gov [Internet]. (Bethesda (MD) : National Library of Medicine (US) 2000-.

192. St. John's Mercy Research Institute SLICgI. Stereotactic Body Radiotherapy for Liver Tumors. (Bethesda (MD) : National Library of Medicine (US) 2000-.

193. Hamburg-Eppendorf U. Chemoradiotherapy for Patients With Oligometastatic Colorectal Cancer (OLGA). In: ClinicalTrials.gov [Internet]. (Bethesda (MD) : National Library of Medicine (US) 2000-.

194. Center SKCC. Stereotactic Radiation Therapy for Pediatric Sarcomas. In: ClinicalTrials.gov [Internet]. (Bethesda (MD) : National Library of Medicine (US) 2000-.

195. Niibe Y, Hayakawa K. Oligometastases and oligo-recurrence : the new era of cancer therapy. Japanese journal of clinical oncology. 2010; 40:107-111.

196. Niibe Y, Chang JY. Novel insights of oligometastases and oligo-recurrence and review of the literature. Pulmonary medicine. 2012; 2012:261096.

197. Palma DA, Salama JK, Lo SS, Senan S, Treasure T, Govindan R, Weichselbaum R. The oligometastatic state - separating truth from wishful thinking. Nature reviews Clinical oncology. 2014; 11:549-557.

198. Mohler JL, Kantoff PW, Armstrong AJ, Bahnson RR, Cohen M, D'Amico AV, Eastham JA, Enke CA, Farrington TA, Higano CS, Horwitz EM, Kawachi MH, Kuettel M, Lee RJ, Macvicar GR, Malcolm AW, et al. Prostate cancer, version 1.2014. Journal of the National Comprehensive Cancer Network : JNCCN. 2013; 11:1471-1479. 